\title{
EuroNeuro 2005
}

\section{Abstracts and Programme}

Cambridge, UK

September 1-4, 2005

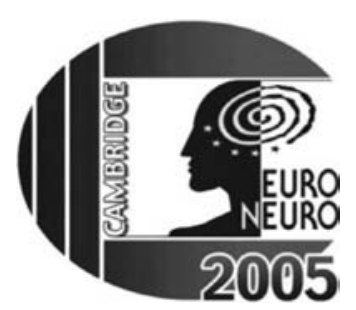


https://doi.org/10.1017/S0265021505001365 Published online by Cambridge University Press 


\section{Welcome}

It is a pleasure to welcome you to Cambridge and EuroNeuro 2005, the fourth international interdisciplinary congress in acute clinical neuroscience. This conference builds on the excellent foundations established in earlier meetings held at Ghenk and Munich. The pace of scientific development in neuroscience has accelerated over the last decade, and recent advances in basic neurobiology are likely to become increasingly relevant to our clinical practice. Importantly, regardless of the parent discipline involved, there is enormous commonality in the knowledge base required to care for the acutely ill patient with neurological disease.

As with previous conferences, EuroNeuro provides a unique opportunity for clinicians from different backgrounds to interact with each other and with basic scientists. We hope and believe that these interactions will provide insights that improve our knowledge of diseases as varied as head injury, stroke and peripheral neuropathy. Importantly, we also plan to stimulate discussion in many areas of basic neurobiology that are likely to be relevant across several diseases, such as the impact of genotype on disease and the assessment of clinical outcome. We have chosen a variety of formats to achieve our aims, including conventional talks, debates, workshops and, for the first time, skill stations that provide hands on training in specific areas.

Finally, the conference also offers you the opportunity to sample the delights of Cambridge, a historic university city with architecture, good food and entertainment.

Again, welcome to Cambridge for what we trust will be a very enjoyable and professionally rewarding experience.
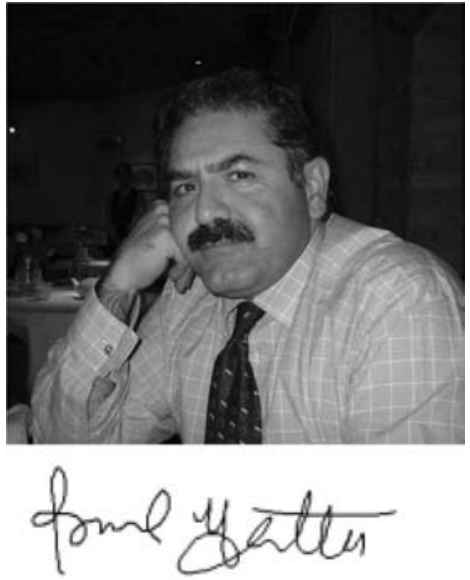

Basil Matta

Clinical Director
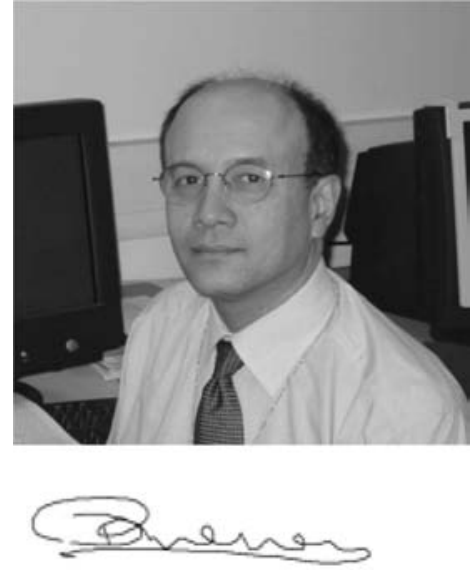

David K Menon

Professor of Anaesthesia 


\section{Committees}

\section{Local Organising Committee}

Basil F Matta, Department of Anaesthesia, Addenbrooke's Hospital, Cambridge David K Menon, Division of Anaesthesia, University of Cambridge, Cambridge

Doris A Chatfield, Division of Anaesthesia, University of Cambridge, Cambridge

\section{Scientific Programme Committee}

David K Menon, Division of Anaesthesia, University of Cambridge, Cambridge Basil F Matta, Department of Anaesthesia, Addenbrooke's Hospital, Cambridge Anthony Absalom, Department of Anaesthesia, Addenbrooke's Hospital, Cambridge Jean-Claude Baron, Stroke Medicine, University of Cambridge, Cambridge Arun Gupta, Department of Anaesthesia, Addenbrooke's Hospital, Cambridge Nick Higgins, Department of Radiology, Addenbrooke's Hospital, Cambridge Peter Hutchinson, Department of Neurosurgery, Addenbrooke's Hospital, Cambridge Peter Kirkpatrick, Department of Neurosurgery, Addenbrooke's Hospital, Cambridge Colin Watts, Brain Repair Centre, University of Cambridge, Cambridge

\section{Conference Secretariat}

Doris A Chatfield, Division of Anaesthesia, University of Cambridge, Cambridge Jackie Jenkins, Wolfson Brain Imaging Centre, University of Cambridge, Cambridge

Event organisation by Global Event Services. 


\section{General Information}

\section{Badges}

Upon registration you will receive a personal badge and conference information. Please wear your badge at all times during the conference as you may be declined entry to the meeting rooms if you do not have your badge visible.

\section{Certificate of Attendance}

A certificate of attendance is included in your participant envelope which will be in your conference bag.

\section{Coffee and Lunch Breaks}

Coffee and lunch will be served in the Guild Hall, Market Square. This is directly adjacent to the Corn Exchange. Please note that you will be required to produce a lunch ticket in order to receive your lunch bags. You will receive a separate ticket if you have informed us of specific dietary requirements.

\section{Conference Language}

The official language of the conference is English. No translation service is provided.

\section{First Aid}

Please return to the registration desk if you require first aid. The telephone number for emergency services (medical, police and fire) in the UK is 999.

\section{Information}

If you require assistance at any time please go to the registration desk in the Corn Exchange foyer. Assistants will be clearly visible in EuroNeuro2005 blue polo shirts.

\section{Internet Access}

Internet access will be available at the Guild Hall.

\section{Meetings}

The meeting will be held at three sites; New Hall, University of Cambridge, Huntingdon Road; Queen's College, University of Cambridge, Silver Street; and the Corn Exchange, Wheeler Street, Cambridge. You will find directions to all the venues in the maps found in your delegate bags.

\section{Registration Desk}

The registration desk will be open from $12.30 \mathrm{pm}$ on Thursday $1 \mathrm{st}$ September for the duration of the meeting. For those attending skills station sessions at New Hall a separate registration desk will be available on site.

\section{Speaker Rooms}

The speaker facilities are located in the King's room at the Corn Exchange. Computer terminals and internet access will be provided for speakers. An assistant will be available for questions. Speakers must bring their lectures to this rooms at least three hours before their presentation, on CDs, floppy disks, or USB storage devices.

\section{Transport}

Cambridge is well served by buses and taxis. The bus services are run by Stagecoach and you will have received details of the routes around the city centre in your conference bags. Tickets may be purchased on the bus. Day rider tickets are usually better value than return journey tickets. You may also wish to consider buying a week's ticket which allows you to board as many times as you wish within the dates on the ticket. There are also city tour buses which will take you on guided tours of the historical city. Please note that a weekly ticket does not entitle you to use the tour buses. 


\section{Social Programme}

\section{Opening Reception}

Guild Hall, Market Square, Cambridge

Thursday 1st September 7.30-9.00 pm

Please join us for the opening ceremony and exhibition at the Guild Hall, Market Square. Light refreshments will be served.

\section{Conference Dinner}

King's College, University of Cambridge

Friday 2nd September $7.30 \mathrm{pm}$

In a city rich in architectural diversity, King's College deserves the royal title. The screens and gatehouse, which separate the college from King's Parade, are early Victorian, to the west, the Gibbs Building and to the south is the dining hall, topped with lanterns, which provides the magnificent setting for our Gala Dinner.

The dining hall has been host to famous alumni including Rupert Brooke, John Maynard Keynes, E. M. Forster, Salman Rushdie and Alan Turing.

Join us beneath the spectacular vaulted ceiling of King's Hall for a sumptuous meal, accompanied by superb wine and company!

If you have not booked a place but would like to attend please check at the registration desk for any late availability.

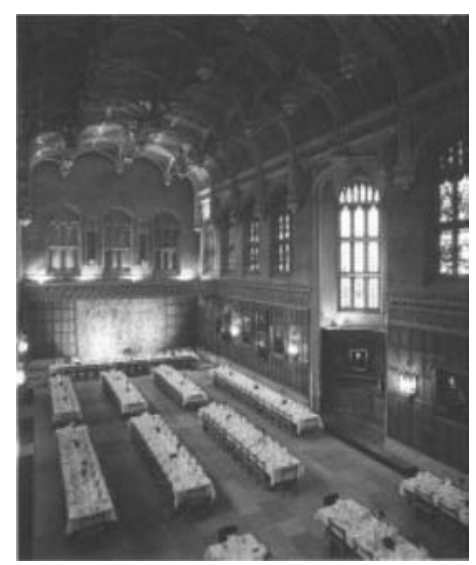


Thursday 1st September 2005

12:30-17:00 General registration open at the Corn Exchange

Registration at New Hall will be available for skills station attendees only

Skills Stations Program

New Hall College, Cambridge

13:30-15:00 Session One

15.00-15:30 Coffee

15:30-17:00 Session Two

Skills Station One: Transcranial Doppler, supported by DWL

Teaching Room, Kaetsu Centre, New Hall College

Moderators: Marek Czosynka, Arthur Lam, Luzius Steiner, Carol Turner

Skills Station Two: Microdialysis, supported by CMA

Seminar Room (a), Buckingham House, New Hall College

Moderators: Peter Hutchinson, Mark O'Connell

Skills Station Three: Brain tissue oxygenation, supported by Codman \& Licox

Seminar Room (b), Buckingham House, New Hall College

Moderators: Arun Gupta, Jurgens Nortje, Ivan Timofeev

Skills Station Four (one): C-Spine for the anaesthetist

Ante Room, New Hall College

Moderator: Mike Todd

Skills Station Four (two): C-Spine for the neurosurgeon

Ante Room, New Hall College

Moderator: Rodney Laing

Skills Station Five: Brain Imaging

Syndicate Room 1, New Hall College

Moderators: Jonathan Coles, David Menon

Skills Station Six: Stealth, supported by Medtronic

Meeting Room, Kaetsu Centre, New Hall College

Moderator: Colin Watts

Skills Station Seven: Imaging support for decisions on thrombolysis in acute ischaemic stroke Syndicate Room 2, New Hall College

Moderators: Jean Claude Baron (one) \& Alistair Buchan (two)

Skills Station Eight: Getting your paper accepted, supported by Cambridge University Press

Music Room, New Hall College

Moderator: David Warner

Following the end of the second skills station coffee will be available at New Hall

Thursday 1st September 2005

17:45-19:15 Industrial symposia

Industrial symposium 1: Corn Exchange

Remifentanil for neuro-anaesthesia and ICU sedation

Sponsored by GlaxoSmithKline 
17:45-18:10

18:10-18:35

18:35-19:00

19:00-19:15

17:45-18:05

18:05-18:25

18:25-18:45

18:45-19:05

19:05-19:15

$17: 45-18: 10$

18:10-18:35

18:35-19:00

19:00-19:15

19:30-21:00
Remifentanil for neuro-anaesthesia: food for thought

Luc de Baerdermaeker, University Hospital, Ghent, Belgium

Remifentanil: protocols, pumps, practicalities

Nigel Huggins, Queen Elizabeth Medical Centre, Birmingham, UK

Remifentanil for neuro-ICU sedation

Andrew Griffiths, Middlesbrough, UK

Discussion

Industrial symposium 2: Old Hall, Queen's College

Clinical brain tissue monitoring

Sponsored by Codman and CMA

Debate: Tissue monitoring has crossed the threshold from an interesting research tool to a useful clinical tool

\section{The benefits of microdialysis}

Urban Ungerstedt, Karolinska Institutet, Stockholm, Sweden

The cons of microdialysis

Michael De Georgia, The Cleveland Clinic Foundation, Ohio, USA

The benefits of tissue monitoring

Andreas Unterberg, Heidelberg University, Heidelberg, Germany

The cons of tissue monitoring

Nino Stocchetti, University of Milan, Milan, Italy

Discussion

Industrial symposium 3: Fitzpatrick Hall, Queen's College

Fluid therapy for the sick brain

Sponsored by Fresenius-Kabi

\section{Sodium chloride poisoning}

Monty Mythen, University College London, London, UK

Hypertonic solutions and raised intracranial pressure

Peter Andrews, Intensive Care Medicine, University of Edinburgh, UK

Safety of the medium substitution starches in brain injury

Thomas Neff, University Hospital of Zurich, Zurich, Switzerland

Discussion

OPENING RECEPTION AT THE GUILD HALL, MARKET SQUARE, CAMBRIDGE

\section{Friday 2nd September 2005}

08:00-09:30 Problem based discussions

\section{Discussion 1: Corn Exchange}

Where do I set my CPP target in head injury?

Chairs: Basil Matta, Addenbrooke's Hospital, Cambridge, UK

Frank Rasulo, University Hospital of Brescia, Brescia, Italy

Claudia Robertson, Baylor College of Medicine, Houston, Texas, USA

Per-Olof Grande, University of Lund, Lund, Sweden

Luzius A Steiner, University Hospital Basel, Basel, Switzerland

Discussion

08:50-09:15

09:15-09:30

Discussion 2: Fitzpatrick Hall, Queen's College

Electrical storms in the neuro critical care unit - types, detection, management Chairs: Tony Absalom, Addenbrooke's Hospital, Cambridge, UK

Nicholas Bruder, CHU de la Timone, Marseille, France

08:00-08:25 Spreading depression

Anthony J Strong, King's College Hospital, London, UK

08:25-08:50

08:50-09:15 Epidemiology and detection

Paul Vespa, UCLA School of Medicine, Los Angeles, USA

Management

Thomas P Bleck, University of Virginia, Charlottesville, Virginia, USA

09:15-09:30 
Discussion 3: Old Hall, Queen's College

The weak patient in neuro critical care unit

Chairs: Atul Swami, Addenbrooke's Hospital, Cambridge, UK

Martin Smith, University College London Hospital, London, UK

08:15-08:45 The patient who develops weakness late in his ICU stay

Nicola Latronico, University of Brescia, Brescia, Italy

08:45-09:15 Weaning the patient with neuromuscular failure

09:15-09:30 Discussion

10:00-12:30

Poster presentations at Guild Hall and Fisher Hall, Market Square, Cambridge

Fisher Hall

10:00-11:15

Group A: Neuroanaesthesia

Group B: Monitoring and Pathophysiology I

$11: 15-12: 30$

Group C: Clinical Interventions

Group D: Monitoring and Pathophysiology II

Guild Hall

10:00-11:15

Group E: Clinical Practice

Group F: Experimental Medicine and Pathophysiology

$11: 15-12: 30$

Group G: Disease Mechanisms

Group H: Imaging

12:30-14:00

LUNCH and EXHIBITION, Guild Hall, Market Square, Cambridge

14:00-17:30

MULTIDISCIPLINARY SYMPOSIUM 1: Corn Exchange

NEUROLOGICAL OUTCOMES (Part 1)

Chairs: Martin Smith, University College London Hospital, London

Tom Bleck, University of Virginia, Charlottesville, Virginia, USA

14:00-14:25 Effect of genotype on outcome

James Nicoll, University of Southampton, Southampton, UK

14:25-14:50 Effect of neurocritical care on outcome

Mike Diringer, Washington University, St Louis, USA

Impact of health care delivery systems on outcome

14:50-15:10 Stroke: rapid referral for thrombolysis

Alistair Buchan, University of Oxford, Oxford UK

15:10-15:30 System analysis on a population-based level in Southern Bavaria on the prehospital \& early clinical care in severe head Injury

Alex Baethmann, Klinikum of University of Munich, Munich, Germany

15:30-16:00 COFFEE BREAK - Guild Hall, Market Square, Cambridge

\section{NEUROLOGICAL OUTCOMES (Part 2)}

Chairs: David Menon, University of Cambridge, Cambridge, UK

Nino Stocchetti, Universita di Milano, Ospedale, Milan, Italy

16:00-16:30 Approaches to measuring detailed neurocognitive outcomes Barbara Sahakian, University of Cambridge, Cambridge, UK

16:30-17:00 Outcome measures for clinical trials: problems and solutions Lindsay Wilson, Stirling University, Stirling, UK

17:00-17:30 The patient's viewpoint: led by Barbara Wilson and David Menon 


\section{Saturday 3rd September 2005}

\section{8:00-09:30 Breakfast Panels}

Breakfast Panel 1: Corn Exchange

Pharmacological neuroprotection - a forgotten dream?

Chairs: Cathy De Deyne, Critical Care Medicine, Genk, Belgium

Patrick Ravussin, Sion Hospital, Sion, Switzerland

08:00-08:25 Anaesthetic neuroprotection

Christian Werner, Johannes Gutenberg-Universitat Mainz, Mainz, Germany

08:25-08:50

Update on pharmacological neuroprotection

Peter Andrews, Intensive Care Medicine, University of Edinburgh, Edinburgh, UK

08:50-09:15

Update on the CRASH trial

09:15-09:30

lan Roberts, London School of Hygiene \& Tropical Medicine, London, UK

Discussion

Breakfast Panel 2: Fitzpatrick Hall, Queen's College

Integrating bedside monitoring in acute neurological insults

Chairs: Arun Gupta, Addenbrooke's Hospital, Cambridge, UK

Pol Hans, University Hospital, Liege, Belgium

08:00-08:25 ICP: more than a number

Marek Czosnyka, University of Cambridge, Cambridge, UK

08:25-08:50

Integrating multimodal monitoring in the ICU

Juan Sahuqillo, Vall d'Hebron University Hospital, Barcelona, Spain

08:50-09:15

What does hyperoxia tell us about brain chemistry in trauma

Nino Stocchetti, Ospedale Maggiore Policlinico, Milano, Italy

09:15-09:30

Discussion

Breakfast Panel 3: Old Hall, Queen's College

Interventional neuroradiology in the neuro critical care unit patient

Chairs: Nick Higgins, Addenbrooke's Hospital, Cambridge, UK

Basil Matta, Addenbrooke's Hospital, Cambridge, UK

08:00-09:00 Mini debate: Surgery for aneurysmal SAH - a failed therapy

1. For the motion: Robin Sellar, Division of Neuroscience, University of Edinburgh, Edinburgh, UK

2. Against the motion: Peter Kirkpatrick, Addenbrooke's Hospital, Cambridge, UK

3. Discussion from the floor

09:30-10:00

10:00-13:00

COFFEE BREAK - Guild Hall, Market Square, Cambridge

MULTIDISCIPLINARY SYMPOSIUM 2: (Part 1) Corn Exchange HYPOTHERMIA - THEORY, PRACTICE, PITFALLS \& APPLICATIONS

Chairs: Mike Todd, University of lowa Health Care, lowa City, USA

Peter Hutchinson, Addenbrooke's Hospital, Cambridge, UK

10:00-10:20 Theory: Mechanisms of hypothermic neuroprotection

Alistair Buchan, University of Oxford, Oxford, UK

10:20-10:40 Practice: Inducing hypothermia in the clinical setting

Mike Diringer, Washington University, St Louis, USA

10:40-11:00 Pitfalls: Side effects of hypothermia in critically ill patients

Kees Polderman, Vrije University Medical Center, Amsterdam, The Netherlands

11:00-11:30 COFFEE BREAK - Guild Hall, Market Square, Cambridge

MULTIDISCIPLINARY SYMPOSIUM 2: (Part 2) Corn Exchange APPLICATIONS

Chairs: Peter Andrews, University of Edinburgh, Edinburgh, UK

Basil Matta, Addenbrooke's Hospital, Cambridge, UK

11:30-11:50 $\quad$ (a) Cardiac arrest

Bernd Bottiger, University of Heidelberg, Heidelberg, Germany

11:50-12:10 $\quad$ (b) Stroke

Stefan Schwab, University of Heidelberg, Heidelberg, Germany 
$12: 10-12: 30 \quad$ (c) Subarachnoid haemorrhage

Mike Todd, University of lowa Health Care, lowa City, USA

12:30-12:50 $\quad$ (d) Head injury

Kees Polderman, Vrije University Medical Center, Amsterdam, The Netherlands

12:50-13:00

(e) Discussion

13:00-14:30 LUNCH and EXHIBITION, Guild Hall, Market Square, Cambridge

13:00-14:30 Industry Lunchtime Symposium. Corn Exchange

Approaches to anaesthesia for the 'at risk' central nervous system

Sponsored by Abbott

Chairs: Hugo van Aken, Universitätsklinikum Münster, Münster, Germany

Patrick Ravussin, Sion Hospital, Sion, Switzerland

13:00-13:25 Does choice of anaesthesia affect outcome?

Cathy De Deyne, Ziekenhuis Oost-Limburg, Ghenk, Belgium

13:25-13:50 Neurosurgery without general anaesthesia

Pol Hans, University Hospital, Liege, Belgium

13:50-14:15 Does monitoring make a difference?

Cor Kalkman, University Medical Center, Utrecht, The Netherlands

$14: 15-14: 30$

Discussion

14:30-15:50 MULTIDISCIPLINARY SYMPOSIUM 3: (Part 1) Corn Exchange IMAGING REGIONAL PHYSIOLOGY AND LOCAL NEURONAL OUTCOME

Chairs: J D Pickard, University of Cambridge, Cambridge, UK

Mike Diringer, Washington University, St Louis, USA

14:30-14:50 Everyday answers to imaging perfusion: Dynamic CT

Jonathan Gillard, University Department of Radiology, Cambridge, UK

14:50-15:10 Quantitative mapping of ischaemia: Xenon CT

Howard Yonas, University of Pittsburgh, Pittsburgh, USA

$15: 10-15: 30$

Defining ischaemia using PET

Jonathan Coles, University of Cambridge, Cambridge, UK

15:30-15:50 MR quantitation of ischaemic insults

Lief Ostergaard, Aarhus University Hospital, Aarhus, Denmark

15:50-16:30 COFFEE BREAK - Guild Hall, Market Square, Cambridge

MULTIDISCIPLINARY SYMPOSIUM 3: (Part 2) Corn Exchange Chairs: Jonathan Coles, University of Cambridge, Cambridge, UK Lief Ostergaard, Aarhus University Hospital, Aarhus, Denmark

16:30-16:50 Blood brain barrier dysfunction Louis Puybasset, Hopital de la Pitie-Salpetriere, Paris, France

16:50-17:10 The role of MR spectroscopy in quantifying neuronal loss Tom Cadoux-Hudson, John Radcliffe Hospital, Oxford, UK

17:10-17:30 The role of ${ }^{11} \mathbf{C}$-flumazenil PET in defining neuronal outcomes Jean-Claude Baron, University of Cambridge, Cambridge, UK

\section{Sunday 4th September 2005}

08:00-09:15 Prize Presentations. Corn Exchange

Chairs: David Menon, University of Cambridge, Cambridge, UK

Basil Matta, Addenbrooke's Hospital, Cambridge, UK

09:15-10:30 Tomorrow's therapies: Brain repair. Corn Exchange

Chairs: Colin Watts, Brain Repair Centre, University of Cambridge, Cambridge, UK

Rowan Burnstein, Addenbrooke's Hospital, Cambridge, UK 
09:15-09:45

09:45-10:15

10:15-10:30

10:30-11:00

11:00-12:30

11:00-11:25

$11: 25-11: 50$

$11: 50-12: 15$

$12: 15-12: 40$

12:40-13:00

13:00

Augmenting function of the nervous system: The implications of deep brain stimulation Alim-Louis Benabid, University Joseph Fourier De Grenoble, Grenoble, France Repairing the damaged spinal cord: Initiating clinical trials James Fawcett, Brain Repair Centre, University of Cambridge, Cambridge, UK Discussion

COFFEE BREAK - Guild Hall, Market Square, Cambridge

Hot topics: Clinical trials for today \& tomorrow. Corn Exchange

Chairs: Pedro Amorim, Hospital Geral de Santo Antonio, Santo Antonio, Portugal David Menon, University of Cambridge, Cambridge, UK

Decompressive craniectomy: The RESCUE ICP study Peter Hutchinson, University of Cambridge, Cambridge, UK New approaches to assessing outcome in clinical trials Gordon Murray, University of Edinburgh Medical School, Edinburgh, UK Feasibility of clinical trials in paediatric head injury Robert Tasker, Addenbrooke's Hospital, Cambridge, UK Xenon: current clinical status Mervyn Maze, Imperial College, London, UK

\section{Discussion}

\section{CLOSE OF CONFERENCE}




\section{Contents}

\section{ABSTRACTS}

\section{Group A: Neuroanaesthesia}

Neuroprotective use of mild hypothermia for aneurysm surgery

A-2 A comparative study of ICP and CPP in patients with cerebral tumours anaesthetized with propofol/fentanyl or propofol/remifentanil T. Groefte, L. Krogh, K.I. Goldbaekdal, G.E. Cold

A-3 Comparative study of the ICP-reducing effect of hyperventilation, 10 degrees reverse Trendelenburg position, mannitol treatment indomethacin or drainage in patients subjected to craniotomy for cerebral tumors K.I. Golbækdal, M. Rasmussen, N. Juul, B. Dahl, G.E. Cold

A-4 Dexmedetomidine infusion for awake craniotomy H. Bilgin, G. Korfalý, F.N. Kaya, A. Bekar, A. Korfalý

A-5 Effects on mean arterial blood pressure, cerebral perfusion pressure and intracranial pressure after bolus propofol during propofol/fentanyl maintenance anaesthesia

L. Jensen, L. Krogh, B. Dahl, N. Juul, G.E. Cold

A-6 Effects on mean arterial blood pressure, cerebral perfusion pressure and intracranial pressure after bolus propofol or remifentanil during propofol/remifentanil maintenance anaesthesia

L. Jensen, L. Krogh, B. Dahl, N. Juul, G.E. Cold

A-7 Evaluation of anaesthetic technique for deep brain stimulation in Parkinson's disease S. Batista, A.L. Macedo, J. Vaquero, M.R. Orfão, J.M. Nunes

A-8 The influence of brain tumours on BIS response to a remifentanil bolus D.A. Ferreira, C.S. Nunes, L.M. Antunes, P. Amorim

A-9 Survey of neuroanaesthesia practice patterns in Colombia M. Arango, C. Niño, J. Mejia-Mantilla

A-10 Microvascular decompression for trigeminal neuralgia: review of intraoperative complications S. Batista, M. Martins, A.L. Macedo, M.R. Orfão, J.M. Nunes

A-11 EEG spectral entropy correlates with regional cerebral blood flow during sevoflurane and propofol anaesthesia A. Maksimow, K. Kaisti, S. Aalto, M. Mäenpää, S. Jääskeläinen, S. Hinkka, S. Martens, M. Särkelä, H. Viertiö-Oja, H. Scheinin

A-12 The comparison of the neuroprotective effects of isoflurane and sevoflurane on cerebral ischemia E. Sayan, G. Korfali, H. Bilgin, V.M. Bayrak, I.H. Ulus

\section{Group B: Monitoring and Pathophysiology I}

B-1 Continuous EEG and SEP monitoring in 36 comatose patients: prelimary results P. Lavicka, M. Choc, R. Pikner, J. Mracek, R. Bosman, O. Topolcan

B-3 Neurophysiological assessment in severe head trauma

B-4 Is monitoring intracranial pressure in patients with malignant middle cerebral artery infarction useful? M.A. Poca, J. Sahuquillo, M. Riveiro, A. Rovira, P. Delgado, J. Álvarez-Sabín

B-5 The misleading effect of cerebrovascular autoregulation variability on pressure-volume index assessment in clinical practice A. Lavinio, F. Rasulo, E. De Peri, A. Candiani, N. Latronico

B-6 Cerebrovascular autoregulation changes in sepsis syndrome F. Zubani, E. De Peri, F. Rasulo, A. Candiani, N. Latronico

B-7 Non-invasive monitoring of carbon dioxide reactivity: spatially resolved near infrared spectroscopy and transcranial Doppler M. Tisdall, I. Tachtsidis, C.E. Elwell, M. Smith

B-8 Continuous PtO2 and ICP measurements in awake patients with suspected normal pressure hydrocephalus (NPH) - conclusions for the debate on critical cerebral PtO2 thresholds in the ICU setting H. Wessling, C.L. Simosono, M. Escosa, P. de las Heras

B-9 Association between impaired cerebral autoregulation and outcome in severe pediatric traumatic brain injury M.S. Vavilala, S. Muangman, P. Suz, M.S. Kincaid, A.M. Lam

B-10 Cerebral blood flow autoregulation during experimental S. pneumoniae meningitis M. Pedersen, C.T. Brandt, G.M. Knudsen, P. Skinhøj, K. Møller

\section{Group C: Clinical Interventions}

C-1 Efficiency of erythropoietin to reduce blood transfusion during craniosynostosis surgical repair: a preliminary study 
C-3 Pentobarbital versus thiopental in patients with refractory intracranial hypertension. Preliminary results of a pilot study comparing their efficacy in severe traumatic brain injury

J.P. Barcena, J.A.L. Pou, J. Homar, J.M. Abadal, J.M. Raurich, J.I. Juve, B. Castanayer, J. Sahuquillo

C-4 Decompressive craniectomy following traumatic brain injury leads to reduction in intracranial pressure and improves cerebral autoregulation I. Timofeev, M. Czosnyka, P.J. Kirkpatrick, J. Nortje, D.K. Menon, A.K. Gupta, J.D. Pickard, P.J. Hutchinson

C-5 Context-sensitive corticosteroid use in head injury J.G. Outtrim, F. Bernard, D.K. Menon, B.F. Matta

C-6 Effects of acute treatment with Pravastatin on cerebral vasospasm, autoregulation, and delayed ischemic deficits after aneurysmal subarachnoid hemorrhage: a phase II randomised placebo-controlled trial M.Y. Tseng, M. Czosnyka, H. Richards, J.D. Pickard, P.J. Kirkpatrick

C-7 Empirical vs metabolic monitoring based nutritional support in patients with hemorrhagic stroke N.S. Gadgieva, I.N. Leiderman, A.A. Belkin

C-8 The effect of decompressive craniectomy on ICP in patients with traumatic brain injuries T. Skoglund, C. Eriksson-Ritzén, B. Rydenhag

C-9 Refractory posttraumatic intracranial hypertension: treatment with an external lumbar drainage J. Homar, J.M. Abadal, J.A. Llompart, J. Perez, J. Ibáñez

C-10 Decompressive craniectomy in severe traumatic brain injury treated by an ICP targeted therapy M. Olivecrona, M. Rodling-Wah/ström, S. Naredi, L.-O.D. Koskinen

\section{Group D: Monitoring and Pathophysiology II}

D-1 Attempts of improving accuracy of non-invasive ICP assessment

D-2 Non invasive intracranial pressure evaluation by means of transcranial Doppler outside the ICU

D-3 'ICM+': software for on-line analysis of data from bedside monitors in neurosurgical and neurointensive care patients P. Smielewski, M. Czosnyka, S. Piechnik, L. Steiner, S. Momhjan, J.D. Pickard

D-4 Cerebral haemodynamics assessed by transcranial Doppler ultrasonography during orthotopic liver transplant. A preliminary report M. Czosnyka, E.A. Schmidt, D. Tew, J.R. Klink, M. Balestreri, J.D. Pickard, B.F. Matta

D-5 Monitoring and interpretation of intracranial pressure after head injury M. Czosnyka, M. Balestreri, L. Steiner, M. Hiler, P. Smielewski, P. Hutchinson, J.D. Pickard

D-6 Subdural monitoring of ICP during craniotomy in patients without space-occupying lesions L. Krogh, L. Jensen, T. Groefte, B. Bergholt, G.E. Cold

D-7 Venous jugular bulb desaturation episodes during functional hemispherotomies N. Fàbregas, I. Bel, L. Salvador, R. Valero, E. Carrero, J. Rumià

D-8 Correlation between $\mathrm{AVDO}_{2}$ and arterio-venous difference in $\mathrm{CO}_{2}, \mathrm{pH}$, glucose, lactate and oxygen/glucose index in patients subjected to craniotomy

T. Groefte, K.I. Golbaekdal, L. Krogh, G.E. Cold

D-9 Drift analysis of the Bowman regional cerebral blood flow monitor S. Wolf, L. Schürer, C.B. Lumenta

D-10 The radiographic validity of transcranial Doppler cerebral blood flow velocity measurement M.J. Souter, G.W. Britz, M.S. Kincaid, B. Ghodke, A.M. Lam

\section{Group E: Clinical Practice}

E-1 The significance of CT and detailed neurological examination for non-invasive evaluation of intracranial hypertension S.V. Tsarenko, K.T. Guseynova, S.S. Petrikov, V.V. Krylov

E-2 Case report: the use of phenylephrine in the acute mangement of a patient with a suspected norepinephrine metabolism disorder H. Akrawi, R. Lightfoot, C.A. Eynon

E-3 BIS may reflect cerebral perfusion: case report during neuroendoscopic brain surgery P. Amorim, M.P. Alonso, M.J. Freitas, J. Pamplona, R. Pedroza, A. Verdelho, F. Lobo

E-4 Neuroanesthetic practice in Catalonia, results of a National Survey S. Fernández, R. Valero, A. Alcon, N. Fábregas, Grup ANESCAT

E-5 Survey of neuroanaesthesia practice patterns in Colombia M. Arango, C. Niño, J. Mejia-Mantilla

E-6 Post ISAT pattern of management of 953 patients with intracranial vascular lesions in the West of Scotland P. Harrison, I.E. Scott, V. Lochhead

E-7 Patients with cervical discus prolaps being anaesthetised by nurse anaesthetists J. Foden, R. Stjernholm

E-8 Correlation links between the length of peripherial nerves and the rate of their involvement into critical illness polyneuropathy (CIMP) A.A. Belkin, A.M. Alasheev

E-9 Refractory status epilepticus in children: the role of propofol J.P.J. van Gestel, H.J. Blussé van Oud-Alblas, M. Malingré, F.F.T. Ververs, K.P.J. Braun, O. van Nieuwenhuizen

E-10 Can electrocardiogram electrodes replace bispectral index electrodes for monitoring depth of anesthesia? P. Akavipat, K. Dumrongbul, P. Neamnak

\section{Group F: Experimental Medicine and Pathophysiology}

F-1 Effects of recruitment maneuvers on cerebral and systemic hemodynamics in head trauma patients 
F-3 Cerebral blood flow increases during infusion of mannitol in patients with acute bacterial meningitis

F-4 Nitric oxide activity and cerebral blood flow effects of sodium nitroprusside and sodium nitrite

F-5 The impact of open lung ventilation on intracranial pressure in neurosurgical patients S. Wolf, D.V. Plev, L. Schürer, H.A. Trost, C.B. Lumenta

F-6 Intraarterial infusion of nimodipine for the treatment of cerebral vasospasm following subarachnoid hemorrhage (SAH) K. Bülow, J. Nepper-Rasmussen, F. Gaarskjaer

F-7 Prostacyclin treatment of nimodipine resistant cerebral vasospasm - a pilot study L.-O.D. Koskinen, M. Olivecrona, M. Rodling-Wahlström, S. Naredi

F-8 Effect of norepinephrine on brain $\mathrm{SO}_{2}$ compared with dobutamine K. Sato, H. Kamii, H. Shimizu, M. Kato

F-9 Cerebral metabolic improvement following hypertonic saline administration in patients with severe subarachnoid haemorrhage P.G. Al-Rawi, M.-Y. Tseng, J. Nortje, B.F. Matta, P.J. Hutchinson, P.J. Kirkpatrick

F-10 Effect of ischemic preconditioning on brain tissue gases and $\mathrm{pH}$ during temporary cerebral artery occlusion M.T.V. Chan, R. Boet, S.C.P. Ng, W.S. Poon, T. Gin

\section{Group G: Disease Mechanisms}

G-1 The role of apolipoprotein E polymorphism in the course of subarachnoidal haemorrhage

G-2 Circulating levels of endothelin-1 (ET) in patients with acute bacterial meningitis K. Møller, G. Strauss, F. Tofteng, C. Sahl, T. Qvist, L. Edvinsson

G-3 Brain natriuretic peptide (BNP) is a marker of subarachnoid haemorrhage severity G. Audibert, G. Steinmann, N. de Talance, C. Charpentier, M.H. Laurens, P.M. Mertes

G-4 Apolipoprotein E (APOE) promoter polymorphism A-491T is associated with increased intracranial pressure and reduced cerebral perfusion pressure in human traumatic brain injury J.C. Goodman, M. Van, S.P. Gopinath, A.B. Valadka, C.S. Robertson

G-5 Neurones express macrophage inflammatory protein-2 following traumatic brain injury in the rat J.K.J. Rhodes, P.J.D. Andrews, J. Sharkey

G-6 Slow potential changes sustained by infarcting human brain tissue: significance in the characterisation of spreading depolarisation R. Bhatia, M. Fabricius, P. Hashemi, S. Fuhr, M.G. Boutelle, M. Lauritzen, A.J. Strong

G-7 The lack of prostacyclin effect in $\mathrm{IP}_{2}$ receptor deficient mice increases contusion of the traumatised brain C. Lundblad, P.O. Grände, T. Moska, P. Bentzer

G-8 Lower albumin levels correlate with poor outcome in traumatic brain injury F. Bernard, J. Outtrim, D.K. Menon, B.F. Matta

G-9 Xenon impairs neurocognitive and histologic outcome following cardiopulmonary bypass combined with cerebral air embolism in the rat B. Jungwirth, M.L. Gordan, M. Blobner, W. Schmehl, E.F. Kochs, G.B. Mackensen

\section{Group H: Imaging}

$\mathrm{H}-1 \quad$ Coexisting low cerebral blood flow and volume in head injury is due to microvascular ischaemia, not hypometabolism J.P. Coles, D.K. Menon, T.D. Fryer, D.A. Chatfield, A.K. Gupta, L.A. Steiner, A.J. Johnston, J. Nortje, P.J.A. Hutchinson, F.I. Aigbirhio, J.C. Clark, J.D. Pickard

$\mathrm{H}-2 \quad$ Metabolic brain changes detected by ${ }^{1} \mathrm{H}-\mathrm{MRSI}$ are relevant to clinical severity and outcome in traumatic brain injury S. Marino, E. Zei, M. Battaglini, C. Vittori, P. Bramanti, A. Federico, N. De Stefano

H-3 Long-term outcome of patients with ganglionic hemorrhage: influence of intraventricular hemorrhage and occlusive hydrocephalus S. Nagel, H.B. Huttner, M. Köhrmann, C. Berger, D. Georgiadis, S. Schwab

$\mathrm{H}-4 \quad$ Brain SG of the non contused areas as a marker of severity in human traumatic brain injury V. Degos, T. Lescot, A. Zouaoui, H. Hermann, F. Préteux, P. Coriat, L. Puybasset

$\mathrm{H}-5 \quad$ Diffusion tensor imaging in chronic head injury survivors C.H. Salmond, D.K. Menon, D.A. Chatfield, J.D. Pickard, B.J. Sahakian

$\mathrm{H}-6 \quad$ Structural changes in the caudate nucleus in normal pressure hydrocephalus (NPH) E.E. DeVito, C.H. Salmond, L.K. Tyler, V. Lupson, B.K. Owler, B.J. Sahakian, J.D. Pickard

H-7 The effect of hypertonic saline on cerebral contusion after traumatic brain injury: a tomodensitometric study T. Lescot, V. Degos, A. Zouaoui, C. Fetita, J.-C. Muller, F. Preteux, P. Coriat, L. Puybasset 
https://doi.org/10.1017/S0265021505001365 Published online by Cambridge University Press 


\section{A-1}

Neuroprotective use of mild hypothermia for aneurysm surgery G. Roman ${ }^{1}$, S. Martin ${ }^{2}$

${ }^{1}$ Department of Anesthesiology and Intensive Care, ${ }^{2}$ Department of Neurosurgery, University Hospital Brno, Czech Republic

Introduction: We conducted a prospective study of managed mild hypothermia as a neuroprotective method in patients undergoing surgical treatment of intracranial aneurysm.

Methods: Thirty-eight patients with established intracranial aneurysm (HuntHess score 1 to 3 ) were enrolled to the study group. After introduction of anesthesia, we started cooling with two circulating-water mattresses (Blanketrol III, Cincinnati Sub Zero). Body temperature was maintained at $34^{\circ} \mathrm{C}$ during the preparation of cerebral vessels.

Results: Mean anesthesia time was $358 \pm 49$ minutes, and mean surgery time was $285 \pm 37$ minutes. Blood loss was $430 \pm 130 \mathrm{ml}$. Core body temperature was $37.1 \pm 0.4^{\circ} \mathrm{C}$ during introduction to anesthesia and the lowest core body temperature during surgery was $33.8 \pm 0.4^{\circ} \mathrm{C}$. The core body temperature when the patient was leaving the operating room was $35.8 \pm 0.5^{\circ} \mathrm{C}$. The cooling rate was $0.9 \pm 0.3^{\circ} \mathrm{C} /$ hour and the rewarming rate was $0.7 \pm 0.4^{\circ} \mathrm{C} /$ hour. The required core body temperature was achieved in $100 \%$ of cases. Measured values of both esophageal and bladder temperature were not significantly different $(P=0.4922)$. No significant difference was found when preoperative coagulation parameters and values measured preoperatively during managed hypothermia were compared. Platelet counts before surgery and during preoperative hypothermia were $202 \pm 36$ and $207 \pm 33$, respectively $(P=0.9692)$. Conclusions: Neurological condition was evaluated as good in 35 patients (92\%). Two patients died and one patient was in a vegetative state. When compared with a similar group of patients who underwent surgery in 1998 to 2001 , among whom good treatment results were achieved in $80 \%$ of patients, our final good neurological treatment results increased by $12 \%$ due to the introduction of managed hypothermia.

\section{A-2}

A comparative study of ICP and CPP in patients with cerebral tumours anaesthetized with propofol/fentanyl or propofol/remifentanil

T. Groefte, L. Krogh, K.I. Goldbaekdal, G.E. Cold

Department of Neuroanaesthesia, Århus University Hospital, Denmark

Introduction: To study subdural ICP and CPP during craniotomy in patients with cerebral tumours anaesthetized with propofol/fentanyl (P/F) or propofol/ reminfentanil $(P / R)$.

Methods: Subdural ICP, Mean arterial blood pressure (MABP), CPP, $\mathrm{PaCO}_{2}$ jugular venous saturation (SATV) and $\mathrm{AVDO}_{2}$ were compared in 45 patients scheduled to $\mathrm{P} / \mathrm{F}$ and 43 patients to $\mathrm{P} / \mathrm{R}$. The measurements were performed before and after hyperventilation just before opening of the dura in patients with supratentorial tumours.

Results: No significant differences between groups were found as regards demographic data, neuroradiological data, histopathological diagnosis, $\mathrm{PaCO}$, temperature, SATV, AVDO 2 and ICP. CPP was significantly lower in P/R both before and during hyperventilation (figure 1, mean values are indicated)

\begin{tabular}{|c|c|c|c|c|c|c|c|c|c|c|}
\hline & \multicolumn{5}{|c|}{ Before hyperventilatioon } & \multicolumn{5}{|c|}{ During hyperventilation } \\
\hline & $\begin{array}{r}\text { ICP } \\
\mathrm{m} \\
\end{array}$ & $\begin{array}{r}\text { CPP } \\
\mathrm{nHg}\end{array}$ & $\begin{array}{l}\mathrm{PaCO}_{2} \\
\mathrm{kPa}\end{array}$ & $\begin{array}{l}\text { SATV } \\
\%\end{array}$ & $\begin{array}{l}\mathrm{AVDO}_{2} \\
\mathrm{mmol} / \mathrm{l}\end{array}$ & & $\begin{array}{r}\text { CPP } \\
\mathrm{nHg}\end{array}$ & $\begin{array}{l}\mathrm{PaCO}_{2} \\
\mathrm{kPa}\end{array}$ & $\begin{array}{l}\text { SATV } \\
\%\end{array}$ & $\begin{array}{c}\mathrm{AVDO}_{2} \\
\mathrm{mmol} / \mathrm{l}\end{array}$ \\
\hline $\mathrm{P} / \mathrm{F}$ & 6.8 & 80 & 4.6 & 56.7 & 3.2 & 5.1 & 82 & 3.9 & 50.7 & 3.6 \\
\hline
\end{tabular}

*indicates $p<0.05$ between groups

Conclusions: During craniotomy subdural ICP does not differ between propofol/fentanyl and propofol/remifentanil anaesthetized patients. Cerebral perfusion pressure, however, is significantly lower during the latter anaesthesia.

\section{A-3}

Comparative study of the ICP-reducing effect of hyperventilation, 10 degrees reverse Trendelenburg position, mannitol treatment, indomethacin or drainage in patients subjected to craniotomy for cerebral tumors K.I. Golbækdal, M. Rasmussen, N. Juul, B. Dahl, G.E. Cold Department of Anesthesia, Aarhus University Hospital, Århus C, Denmark Introduction: In this study we compared the ICP-reducing effect of hyperventilation, 10 degrees reverse Trendelenburg position ( $\mathrm{rTp}$ ), mannitol treatment, indomethacin or the effect of drainage of cystic tumor in patients with cerebral tumors subjected to propofol/fentanyl anesthesia.

Methods: From our database concerning subdural ICP measurement we collected consecutive data from 70 patients subjected to craniotomy. The inclusion criteria were a subdural ICP $=10 \mathrm{mmHg}$, combined with increased tension of dura. The following ICP-reducing managements were analyzed: 5 min of hyperventilation $(n=15), 10$ degrees $\operatorname{rTp}(n=15)$, mannitol treatment $0.5-1.0 \mathrm{~g} / \mathrm{kg}(\mathrm{n}=15)$, drainage $17 \pm 9 \mathrm{ml}(\mathrm{n}=13)$ and indomethacin $0.5 \mathrm{mg} / \mathrm{kg}(\mathrm{n}=12)$. Subdural ICP was measured before opening of the dura. The changes in ICP (dICP) and CPP (dCPP) before and after treatment were calculated. The effect of mannitol was analyzed 5 min after mannitol infusion. The effects of $\mathrm{rTp}$, drainage and indomethacin were recorded after one minute. Statistic: Mean \pm SD, ANOVA.

Results: No significant differences as regards demographic data, tumour size, tumour, midline shift, histopathology or dosages of propofol/fentanyl were disclosed. Before treatment no significant inter-group differences in MABP, CPP and ICP were found. The ICP-decreasing effects of drainage, $r \mathrm{p}$, mannitol treatment and indomethacin were significantly greater than that observed with hyperventilation, and drainage was significantly superior to the effect of all other treatments. Compared with rTp and hyperventilation a significant improvement of CPP was observed with mannitol treatment, indomethacin and drainage.

Conclusion: The ICP-reducing effect of drainage is superior to hyperventilation, 10 degrees rTp, mannitol treatment or indomethacin. No differences were disclosed between 10 degrees rTp, mannitol treatment or indomethacin, but these treatment modalities were all superior to hyperventilation.

\section{A-4}

\section{Dexmedetomidine infusion for awake craniotomy}

H. Bilgin ${ }^{1}$, G. Korfalý1, F.N. Kaya ${ }^{1}$, A. Bekar², A. Korfalý2

${ }^{1}$ Departments of Anaesthesiology, and ${ }^{2}$ Neurosurgery, Uludağ University Medical Faculty, Bursa, Turkey

Introduction: Anaesthetic techniques for awake craniotomy include local anaesthesia and sedation or asleep-awake-asleep techniques $[1,2]$. The advantage of awake techniques is the ability to assess the patient's neurological status during surgery, although this benefit has to be balanced against the loss of control of ventilation and assurance of immobility. In this prospective study, we evaluated the effects of different anaesthetic combinations on providing haemodynamic stable, safe airway, and awake patient for neurological tests during awake craniotomy.

Methods: After obtaining ethics committee approval and informed consent, patients scheduled for awake craniotomy were randomly assigned to three groups. Group PF $(\mathrm{n}=17)$ received propofol infusion $\left(2 \mathrm{mg} \mathrm{kg}^{-1} \mathrm{~h}^{-1}\right)$ with bolus fentanyl $\left(1 \mu \mathrm{g} \mathrm{kg}^{-1}\right)$ and repeated as needed; group PR $(\mathrm{n}=17)$ received propofol $\left(2 \mathrm{mg} \mathrm{kg}^{-1} \mathrm{~h}^{-1}\right)$ with remifentanil $\left(0.05 \mu \mathrm{g} \mathrm{kg}^{-1} \mathrm{~min}^{-1}\right)$ infusions; group DF $(n=17)$ received dexmedetomidine infusion (initial dose $1 \mu \mathrm{g} \mathrm{kg}^{-1}$ over $10 \mathrm{~min}$ fallow by of $0.1 \mu \mathrm{g} \mathrm{kg}^{-1} \mathrm{~h}^{-1}$ ) and bolus fentanyl $\left(1 \mu \mathrm{g} \mathrm{kg}^{-1}\right)$ administared as needed. The target level of sedation was 3 of the Modified Observer's Assessment of Alertness/Sedation scale at which patient responds after name is called. Mean arterial pressure (MAP), heart rate $(\mathrm{HR})$, respiratory rate $(\mathrm{RR})$, peripheral oxygen saturation $\left(\mathrm{SpO}_{2}\right)$, endexpiratory carbon dioxide $\left(\mathrm{ETCO}_{2}\right)$ were assessed before $(\mathrm{T} 1)$ and after drug infusion (T2), after scalp infiltration with prilocaine (T3), at skin incision (T4), bone flap removal (T5), dura incision (T6), tumour resection (T7), dura closure (T8), bone flap closure (T9), skin closure (T10), and at the end of surgery (T11). The incidence of complications, infusion rates of drugs, and total dose of local anaesthetic were recorded. Statistical analysis included Wilcoxon signed ranks test, Mann-Whitney U-test and Chi-Square test.

Results: There were no statistically significant differences between the groups regarding the quality of anaesthesia. Total dose of local anaesthetic was lower in group $D$ than in group PR $(p<0.05)$. The total dose of propofol was similar in group PF and PR. The total dose of fentanyl was lower in group DF than in group PF $(p<0.001)$. Peroperative complications such as nausea and vomiting were similar among the groups but the incidence of seizure was higher in group PF $(p<0.05, p<0.05)$. RR was lower in group PR than in group $\mathrm{D}$ at $\mathrm{T} 4$ and $\mathrm{T} 10(\mathrm{p}<0.05, \mathrm{p}<0.05)$. $\mathrm{ETCO}_{2}$ concentration in group PR was higher at T4, T10 than in group D and at T2 than in group PF $(p<0.05$, $\mathrm{p}<0.05, \mathrm{p}<0.05) . \mathrm{SpO}_{2}$ was lower in group $\mathrm{PF}$ at $\mathrm{T} 4$ than in group $\mathrm{D}$ $(p<0.05)$. MAP was lower in group PR at T7 than in group PF $(p<0.05)$. Conclusions: Requirements of opioid and local anaesthetic are decreased by dexmedetomidine. Dexmedetomidine infusion provides sedation without the loss of control of ventilation. We conclude that the use of dexmedetomidine 
in patients undergoing awake craniotomy is safe, comfortable, and easy technique.

\section{References:}

1 Danks RA et al. Patient tolerance of craniotomy performed with the patient under local anesthesia and monitored conscious sedation. Neurosurgery 1998; 42: 28-35.

2 Simon G, Osborn I. Scalpblock and the laryngeal mask hand in hand for awake craniotomies. J Neurosurg Anesthesiol 2004; 16: 364-365.

\section{A-5}

Effects on mean arterial blood pressure, cerebral perfusion pressure and intracranial pressure after bolus propofol during propofol/fentanyl maintenance anaesthesia

L. Jensen, L. Krogh, B. Dahl, N. Juul, G.E. Cold

Department of Neuroanaesthesia, University Hospital of Århus, Denmark

Introduction: Propofol induces a fall in cerebral blood flow (CBF) and cerebral metabolic rate (CMR02). It is unknown whether a propofol bolus dose has the same effect during maintenance anasthesia with propofol/fentanyl. We tested the hypothesis that a propofol dose decreases intracranial pressure (ICP) in patients scheduled for craniotomy during propofol/fentanyl anaesthesia.

Methods: In 11 patients scheduled for craniotomy for cerebral tumours, subdural ICP, mean arterial blood pressure (MAP) and cerebral perfusion pressure (CPP) were monitored every minute for five minutes after a bolus propofol ( $10 \%$ of maintenance dose pr. hour).

Venous jugular saturation $\left(\mathrm{SjO}_{2}\right)$ and $\mathrm{AVDO}_{2}$ was measured at time zero and five minutes after propofol injection. 15 patients served as control.

Results: No difference in demographic data, preoperative blood pressure, $\mathrm{PaCO}_{2}$, rectal temperature, neurological data (tumor size, midline shift, localisation of tumour) or pathohistology of the tumours were disclosed.

The propofol dose (averaging $51 \mathrm{mg}$ ) induced a significant fall in MABP and CPP lasting from one minute after administration and the following 4 minutes. The maximum fall in MABP and CPP averaged 6.9 and $6.8 \mathrm{mmHg}$ respectively. ICP did not change significantly.

In the control group MABP, CPP and ICP were unchanged. In both groups $\mathrm{SjO}_{2}$ and $\mathrm{AVDO}_{2}$ were unchanged.

Conclusion: A bolus dose of propofol administered during propofol/ fentanyl anaesthesia does not change ICP, $\mathrm{SjO}_{2}$ or $\mathrm{AVDO}_{2}$ but reduces MABP and CPP significantly.

\section{A-6 \\ Effects on mean arterial blood pressure, cerebral perfusion pressure and intracranial pressure after bolus propofol or remifentanil during propofol/remifentanil maintenance anaesthesia}

L. Jensen, L. Krogh, B. Dahl, N. Juul, G.E. Cold

Department of Neuroanaesthesia, University Hospital of Århus, Denmark

Introduction: Propofol induces a fall in cerebral blood flow (CBF) and cerebral metabolic rate (CMR02) accompanied by a decrease in intracranial pressure (ICP). It is unknown whether a bolus dose of propofol or remifentanil has the same effect during maintenance anaesthesia with propofol/remifentanil. We tested the hypothesis that a bolus dose of propofol or remifentanil decreases intracranial pressure (ICP) in patients scheduled for craniotomy during propofol/remifentanil anaesthesia.

Methods: Three groups of 15 patients each were studied. A control group, a group where $10 \%$ of the maintenance dose per hour of propofol was given as a bolus dose, and a group where $10 \%$ of the remifentanil maintenance dose per hour was given.

All patients were scheduled for craniotomy for cerebral tumours. Subdural ICP, mean arterial blood pressure (MABP) and cerebral perfusion pressure (CPP) were monitored every minute for five minutes after a bolus of either propofol or remifentanil.

$\mathrm{PaCO}_{2}$, venous jugular saturation $\left(\mathrm{SjO}_{2}\right)$ and $A V D O_{2}$ was measured at time zero and five minutes after bolus injection.

Results: No difference in demographic data, preoperative blood pressure, $\mathrm{PaCO}_{2}$, rectal temperature, neurological data (tumor size, midline shift, localisation of tumour) or pathohistology of the tumours were disclosed.

The propofol dose (averaging $47 \mathrm{mg}$ ) induced a significant fall in MABP and CPP lasting from one minute after administration and the following 4 minutes.

Likewise remifentanil (averaging 188 mikrog) induced a significant fall in MABP and CPP lasting 4 minutes.
In all groups ICP was unchanged, and MABP and CPP were unchanged in the control group.

In all groups $\mathrm{SjO}_{2}$ and $\mathrm{AVDO}_{2}$ were unchanged during the 5 minute study period.

Conclusion: A bolus dose of propofol or remifentanil administered during propofol/remifentanil anaesthesia does not change ICP, $\mathrm{SjO}_{2}$ or $\mathrm{AVDO}_{2}$ but reduces MABP and CPP significantly.

\section{A-7 \\ Evaluation of anaesthetic technique for deep brain stimulation in Parkinson's disease}

S. Batista, A.L. Macedo, J. Vaquero, M.R. Orfão,

J.M. Nunes

Department of Anaesthesiology, University Hospital of Coimbra, Portugal

Introduction: Deep brain stimulation is a new treatment option for Parkinson's disease. The aim of our study was to evaluate the anaesthetic technique and intraoperative adverse events.

Methods: We have performed a retrospective review of anaesthetic records from patients underwent stereotactic microelectrode-guided deep brain surgery under sedation. Variables recorded were age, sex, quality of patient cooperation, anaesthetic drugs, duration of surgery and anaesthetic complications. Results: Ten patients ( 4 women, 6 men) with a mean ( \pm SD) age of $54 \pm 9.6$ years underwent the procedure from 2004 through 2005. The duration of surgery was $13.3 \pm 3.1$ hours. We have used propofol in TCl in all patients, local anaesthetics and non-opioids analgesics. The most common complications involved hemodynamics: arterial hypertension and tachycardia, well managed with labetolol. In one patient the surgery was interrupted after placement of the first electrode because there were hypercapnia, hypertension and agitation; he was submitted to new surgery one week later without any complications. Long term complications included: cerebral hematoma without surgical indications, rejection of material and behaviour disturbs. All patients had good improvement of Parkinson symptoms.

Conclusions: Deep brain stimulation requires surgery of long duration demanding patient cooperation. Good selection and motivation of these patients are very important.

Careful clinical monitoring the patient and cooperation with the surgical team are necessary during the procedure to minimize cardiovascular events. Propofol $(\mathrm{TCl})$ is a suitable option, without interfering in the intraoperative neurophysiological evaluation.

\section{A-8}

\section{The influence of brain tumours on BIS response to a remifentanil bolus}

D.A. Ferreira, C.S. Nunes, L.M. Antunes, P. Amorim

Serviço de Anestesiologia, Hospital Geral de Santo António, Porto, Portugal Introduction: Several studies addressed the effect of remifentanil (Remi) on BIS. To our knowledge, there are no studies about the influence of remifentanil on BIS in patients with brain tumours. We analyzed the effects of a Remi bolus on BIS and haemodynamics in patients with brain tumours.

Methods: GROUP 1: Patients subjected to spinal surgeries, Glasgow 15, ASA I/II. GROUP 2: Patients with cerebral tumours subjected to surgery, Glasgow 15, ASA I/II. Both groups received a $2 \mu \mathrm{g} / \mathrm{kg}$ Remi bolus under TIVA with TCl for propofol (Prop) [1] and Remi [2] in a period free from stimuli. BIS and haemodynamic data were collected from A-2000XP BIS monitor (every $1 \mathrm{~s})$ and $\mathrm{AS} / 3$ Datex monitor (every $5 \mathrm{~s}$ ). Data was analyzed at the time of Remi bolus (T0), 30 (T30), 60 (T60), 90 (T90) and $120 \mathrm{~s}$ after (T120). BIS data was analyzed using the area under the curve (AUC) calculated for each $30 \mathrm{~s}$ periods before and after T0. Basal AUC (average of the four AUC before Remi bolus) was considered as the baseline. Statistics used Student $t$ - test. Data are mean \pm sd.

Results: GROUP 1: 12 patients, $42.9 \pm 9.9$ years, $64.8 \pm 16.5 \mathrm{~kg}$, $164 \pm 10 \mathrm{~cm}, 9$ females. During the study period, the average Prop effect site predicted concentration (PropCe) was $2.8 \pm 0.5 \mu \mathrm{g} / \mathrm{ml}$. In the two minutes period before Remi bolus, average BIS values were $44.3 \pm 6.8$. The AUC for BIS decreased significantly at T90/120. Mean arterial pressure (MAP) decreased significantly at T90 and T120. Heart rate (HR) decreased significantly at T60, T90 and T120. GROUP 2: 12 patients, $53.9 \pm 13.7$ years, $69.3 \pm 12.1 \mathrm{~kg}, \pm 163 \mathrm{~cm}, 8$ females. During the study period, the average PropCe was $2.7 \pm 0.6 \mu \mathrm{g} / \mathrm{ml}$. In the two minutes period before Remi bolus, average BIS values were $42.1 \pm 7.3$. The AUC for BIS decreased significantly at T60/T90 and at T90/120. MAP decreased significantly at T60, T90 and T120. HR decreased significantly at T90 and T120. There were no differences in HR or MAP between the two groups at any time point. The 
AUC for BIS at T60/90 was significantly lower $(P<0.05)$ in patients with brain tumours when compared to patients without brain tumours; at T90/120 there were no differences in AUC for BIS between the groups.

\begin{tabular}{llccccc}
\hline & & T0 & T0/30 & T30/60 & T60/90 & T90/120 \\
\hline GROUP 1 & RemiCe $(\mathrm{ng} / \mathrm{ml})$ & $1.06 \pm 0.67$ & $4.89 \pm 0.52$ & $7.93 \pm 0.92$ & $8.69 \pm 1.26$ & $8.46 \pm 1.44$ \\
& AUC & $1329 \pm 180$ & $1356 \pm 195$ & $1386 \pm 171$ & $1316 \pm 142$ & $1204 \pm 166^{\mathrm{a}}$ \\
& MAP $(\mathrm{mmHg})$ & $92.9 \pm 13.3$ & $91.1 \pm 13.4$ & $87.4 \pm 13.8$ & $80.4 \pm 13.8^{\mathrm{a}}$ & $72.3 \pm 13.5^{\mathrm{c}}$ \\
& HR $(\mathrm{bpm})$ & $75.5 \pm 13.9$ & $73.8 \pm 13.6$ & $70.8 \pm 11.7^{\mathrm{b}}$ & $67.2 \pm 11.2^{\mathrm{c}}$ & $64.9 \pm 10.9^{\mathrm{c}}$ \\
GROUP 2 & RemiCe $(\mathrm{ng} / \mathrm{ml})$ & $1.65 \pm 0.62$ & $5.41 \pm 0.85$ & $8.66 \pm 0.74$ & $9.75 \pm 0.76$ & $9.73 \pm 0.84$ \\
& AUC & $1264 \pm 188$ & $1238 \pm 214$ & $1270 \pm 202$ & $1154 \pm 189^{\mathrm{a}}$ & $112 \pm 175^{\mathrm{b}}$ \\
& MAP $(\mathrm{mmHg})$ & $101 \pm 19.8$ & $99.8 \pm 16.1$ & $93 \pm 18.6^{\mathrm{b}}$ & $83.2 \pm 15.8^{\mathrm{c}}$ & $77.4 \pm 14.5^{\mathrm{c}}$ \\
& HR (bpm) & $68.8 \pm 16.1$ & $68.3 \pm 15.6$ & $65.2 \pm 11.6$ & $63.3 \pm 14.3^{\mathrm{b}}$ & $61.8 \pm 13^{\mathrm{c}}$ \\
\hline
\end{tabular}

RemiCe: Remifentanil predicted effect site concentration.

ap $<0.05$, bP $<0.01,{ }^{c} P<0.001$.

Conclusions: The decrease in BIS in both groups followed the decrease in MAP. There was no statistically difference in the decrease in MAP between both groups. When comparing the decrease in MAP and the decrease in AUC for BIS between T60 and T90 (first significant decrease) in both groups, there was a decrease of 12 in AUC for each $\mathrm{mmHg}$ of decrease in MAP in Group 2 , and only a decrease of 2 in AUC for each $\mathrm{mmHg}$ of decrease in Map in Group 1. It is possible that the reduction in MAP after remifentanil may have caused a reduction in cerebral perfusion, which could decrease the cerebral electric activity measured by BIS. This happened while MAP was still above "normal" minimum autoregulation levels; the increased intracranial pressure in patients with brain tumours may require higher MAP to keep perfusion. The drop in BIS could reflect a reduction in cerebral perfusion.

References:

1 Schnidero. Anesthesiology 1998; 88: 1170-1182.

2 Minto. Anesthesiology 1997; 86: 24-33.

\section{A-9}

\section{Survey of neuroanaesthesia practice patterns in Colombia}

M. Arango ${ }^{1}$, C. Niño ${ }^{2}$, J. Mejia-Mantilla ${ }^{3}$

${ }^{1}$ Department of Anaesthesia, University of Western Ontario, Canada, ${ }^{2}$ Fundación Médica Santa Fe Bogotá, Colombia, ${ }^{3}$ Fundación Valle de Lili Cali, Colombia

Introduction: Neuroanaesthesia as a subspecialty is a relatively new in Colombia. Our objective was to assess current anaesthesia practice in Colombia with respect to patients undergoing cerebral supratentorial tumour (CST) resection and aneurysm clipping (AC).

Methods: In Nov 2004, a postal questionnaire survey was mailed to 48 hospitals (Level IV-V) throughout Colombia which provided care for adult neurosurgical patients on a routine basis. Questions on practice patterns included type of monitoring, anaesthesia techniques, and use of hypertonic solutions, hyperventilation, systemic hypotension and hypothermia.

Results: $34 / 48$ hospitals responded ( $71 \%$ response rate) by Jan 2005 . Of these, 13/34 (38\%) had established anaesthesia residency programs. However, only $2.9 \%$ of all cases were done by anaesthesiologists with a special interest in neuroanaesthesia. While only $3 / 34$ hospitals had a neurointensive care unit, $94 \%$ used a surgical ICU for recovery instead of a PACU despite the fact that the majority of patients were extubated in the OR $(78 \%$ in CST and $61 \%$ in AC). For patients undergoing CST, we found that invasive arterial blood pressure (74\%) and central venous pressure (CVP) monitoring (85\%) were routinely used, however intracranial pressure (ICP) monitoring was used in only $6 \%$ of the hospitals. The most popular anaesthesia technique used was balanced volatile anaesthesia with opioids $(88 \%)$, less commonly was total intravenous anesthesia (TIVA) (35\%) and least common was volatile only in $6 \%$. No centre used $\mathrm{N}_{2} \mathrm{O}$. None of the centres used induced systemic mild hypothermia, but $88 \%$ administered steroids intraoperatively. $76 \%$ of hospitals used routine hyperventilation and $38 \%$ used induced systemic hypotension during CST. To reduce brain bulk, mannitol $20 \%$ was used in $97 \%$ of all hospitals and hypertonic saline in $32 \%$. Thiopental infusion was used in $71 \%$ to reduce ICP. Intraoperative magnesium measurement and replacement was routinely performed in $6 \%$ of the hospitals. Normal Saline $0.9 \%$ was used in all patients in all hospitals for fluid replacement.

For patients undergoing $\mathrm{AC}$, only $85 \%$ used invasive arterial blood pressure and $73 \%$ used CVP monitoring. Again the most popular anaesthesia technique used was balanced volatile anaesthesia with opioids $(91 \%)$, less commonly was TIVA (36\%) and $6 \%$ reported using $\mathrm{N}_{2} \mathrm{O}$. $44 \%$ of the hospitals reported using induced systemic mild hypotension routinely during AC $9.1 \%$ used intraoperative induced systemic hypothermia and $49 \%$ used thiopental infusions to reduce ICP.

Conclusion: We found that anaesthesia management for CST did not significantly differ from that for AC. Inhalational anaesthesia was the preferred technique. Despite recent literature refuting any improvement in neurological outcome with intraoperative mild hypothermia during $\mathrm{AC}$ [1], it continues to be used. Induced hypotension and hyperventilation are prevalent practices during CST and AC. This survey shows that current neuroanaesthesia practices in Colombia may not follow recommended guidelines. The provision of neuroanaesthesia educational programs may help to improve existing standards. Reference:

1 Todd MM, Hindman BJ, Clarke WR, Torner JC. Mild intraoperative hypothermia during surgery for intracranial aneurysm. N Engl J Med 2005; 352(2): 135-145.

\section{A-10}

Microvascular decompression for trigeminal neuralgia: review of intraoperative complications

S. Batista, M. Martins, A.L. Macedo, M.R. Orfão, J.M. Nunes

Department of Anaesthesiology, University Hospital of Coimbra, Portugal

Objective: To evaluate intraoperative complications of microvascular decompression (MVD) for trigeminal neuralgia (TN) in our hospital.

Methods: We performed a retrospective review of anaesthetic records of MVD operations for TN performed in 69 consecutive patients with a mean age of 64 , SD 13,8 .

Results: General anaesthesia with sevoflurane was the most used technique. Standard monitoring and invasive arterial pressure were used in all patients. The duration of surgery was $3,2 \pm 1,1$ hours.

Cardiovascular events were the most frequent complications: bradycardia, arterial hypotension and tachycardia. All patients were extubated at the end of surgery.

Conclusion: MVD is an option for medically refractory TN, however because most of these patients were old, a carefully preoperative evaluation is very important.

Invasive monitoring is essential to allow early diagnosis of cardiovascular events.

\section{A-11}

EEG spectral entropy correlates with regional cerebral blood flow during sevoflurane and propofol anaesthesia

A. Maksimow ${ }^{1}$, K. Kaisti ${ }^{2}$, S. Aalto ${ }^{3}$, M. Mäenpää ${ }^{4}$, S. Jääskeläinen ${ }^{5}$, S. Hinkka ${ }^{6}$, S. Martens ${ }^{7}$, M. Särkelä ${ }^{7}$, H. Viertiö-Oja ${ }^{7}$, H. Scheinin ${ }^{1}$

${ }^{1}$ Turku PET Centre, and Department of Pharmacology and Clinical Pharmacology, University of Turku, Finland, ${ }^{2}$ Department of Anaesthesiology and Intensive Care, Turku University Hospital, Finland, ${ }^{3}$ Centre for Cognitive Neuroscience, University of Turku, Finland, ${ }^{4}$ Vaasa Central Hospital, Finland, ${ }^{5}$ Department of Clinical Neurophysiology, Turku University Hospital, Finland, ${ }^{6}$ Department of Biostatistics, University of Turku, Finland, ${ }^{7}$ GE Healthcare Finland Oy, Helsinki, Finland

Introduction: The M-ENTROPYTM (GE Healthcare, Helsinki, Finland) index monitoring based on spectral entropy of the electroencephalogram (EEG) is a promising new method to measure the depth of anaesthesia. We examined the association between spectral entropy and regional cerebral blood flow (rCBF) in healthy subjects anaesthetised with a volatile or an intravenous anaesthetic agent.

Methods: The Hospital Ethics Committee approved the study protocol. Midfrontal-central and posterior temporal-occipital EEG was continuously recorded and spectral entropy from frequency band $0.8-32 \mathrm{~Hz}$ calculated at 0 (awake), 1, 1.5 and 2 minimum alveolar concentration (MAC) of sevoflurane $(n=8)$ or effective plasma concentration 50 (EC50) levels of propofol $(n=6)$ anaesthesia. Each concentration level was maintained for $30 \mathrm{~min}$ and at each levels, $\mathrm{rCBF}$ was assessed using [O-15]-labeled water and positron emission tomography (PET). We looked for correlations between spectral entropy and quantitative rCBF in the frontal cortex and the whole brain. Furthermore, correlations between voxel-level values of relative rCBF and concomitant spectral entropy values were analysed using subject-specific regression analysis and statistical parametric mapping (SPM) software.

Results: Both drugs induced significant $(P<0.001$ for all) cortical and global reductions of $\mathrm{rCBF}$ and spectral entropy. Individual frontal and whole brain $\mathrm{rCBF}$ values correlated with midfrontal-central spectral entropy across the conditions in both groups. A positive correlation of individual frontal cortical rCBF values and midfrontal-central spectral entropy was also found in the sevoflurane group within the 1.5 and $2 \mathrm{MAC}$ concentration levels and in propofol group within the 1.5 EC50 concentration level. Statistically significant group level correlations $(P<0.001)$ were found in the SPM analysis for both anaesthetics. In the sevoflurane group, the associated areas were located bilaterally in large areas of the parietal lobe, and in the propofol group the areas covered widely the frontal and parietal cortices, and parts of the temporal lobes.

Conclusions: $\mathrm{rCBF}$ reductions induced by sevoflurane and propofol anaesthesia associated with spectral entropy of EEG suggesting that this novel measure of anaesthetic depth can depict global changes in neuronal activity induced by the drugs. Interestingly, the cortical areas of the most significant associations were remarkably similar. 


\section{A-12}

\section{The comparison of the neuroprotective effects of isoflurane} and sevoflurane on cerebral ischemia

E. Sayan ${ }^{1}$, G. Korfali', H. Bilgin', V.M. Bayrak', I.H. Ulus ${ }^{2}$

Uludag University, Faculty of Medicine, ${ }^{1}$ Department of Anaesthesiology and Reanimation, ${ }^{2}$ Department of Pharmacology, Bursa, Turkey

Introduction: The aim of this study was to compare the effects of frequently used volatile agents isoflurane and sevoflurane in equipotent anesthetic concentrations on cerebral ischemia by measuring S-100B protein values a marker of ischemia in patients undergoing intracranial tumor surgery [1]. Material: After obtaining the ethics committee approval, 20 ASA grade I-III, patients scheduled for intracranial tumour surgery, were randomized into two groups. A standardized anaesthetic plan was performed with 1.2-2\% end tidal concentration of isoflurane or sevoflurane in $50 \%$ air in oxygen Heart rate, systolic, diastolic and mean arterial pressures, peripheral oxygen saturation, central venous pressure and end tidal concentration of carbon dioxide were measured during surgery. For S-100B protein values, peripheral blood samples were taken at nine different times, during the perioperative period. Mann-Whitney $\mathrm{U}$ and Wilcoxon signed rank test were used for statistical analyses as appropriate.

Results: The demographic data and operation times were similar in both groups. There were no changes in hemodynamic variables, peripheral oxygen saturation or carbon dioxide concentrations with the exception of diastolic pressure following intubation which was decreased in the isoflurane group and increased in sevoflurane group $(P<0.05)$. There were no significant differences in S-100B protein values between the groups.

Conclusion: The current study did not demonstrate a difference in S-100 B protein values in patients undergoing craniotomy using isoflurane or sevoflurane for intraoperative anesthesia. Further work is needed to determine neuroprotective effects of sevoflurane and its effect on S-100B.

Reference:

1 Martens P, Raabe A, Johnsson P. Serum S-100 and neuron-specific enolase for prediction of regaining consciousness after global cerebral ischemia. Stroke 1998; 29: $2363-2369$

\section{Group B: Monitoring and Pathophysiology I}

\section{B-1}

\section{Continuous EEG and SEP monitoring in $\mathbf{3 6}$ comatose patients: prelimary results}

A. Amadori', A. Amantini' ${ }^{2}$, L. Bucciardini' 1 S. Fossi' ${ }^{2}$, A. Grippo ${ }^{2}$, P. Innocenti ${ }^{1}$, F. Pinto ${ }^{2}$

${ }^{1}$ Neurosurgical Intensive Care Unit, ${ }^{2}$ Clinical Neurophysiology Unit, Department of Neurological and Psychiatric Sciences, Azienda Ospedaliera - Universitaria, Careggi - Firenze, Italy

Introduction: we report our experience with continuous neurophysiological monitoring (EEG-SEP) in patients at risk for neurological deterioration. Methods: 36 patients (26 Males and 10 Females, mean age 50) with GCS $<9$ and ICP monitoring were studied. CEEG-SEP monitoring was performed for a period of at least 3 days (mean 8 days, range $3-19$ ): 17 head trauma, $10 \mathrm{SAH}, 8$ intracerebral hemorrage, 1 ischemic stroke. Cycles of CEEG and SEPs from alternate stimulation of both median nerves were performed. We displayed conventional and quantitative EEG (bar-trends of band frequency ratio, automatic calculation of suppression percentage), and SEPs (latency and amplitude trends). SEPs findings were classified into two groups: the "stable" group, when SEPs showed less than $40 \%$ amplitude decrement with a stable sedation, "deteriorated" group when SEPs showed significant modification not due to neurosedation.

Results: A non-convulsive status epilepticus (NCSE) was found in 4 patients monitored only with CEEG. 32 patients underwent a CEEG-SEP: 25 were considered "stable" or improving, taking into account clinical (GCS) and neuroradiological (CT) criteria; neurological deterioration was found in 7 pats ( 2 focal secondary brain damages, 4 diffuse deterioration -3 of whom up to cerebral death, 1 non-neurological death). SEPs were "stable" either when ICP values were considered "safe" either when they were "critical" (20-35 mmHg). In the "deteriorated" group SEPs showed significant alterations that, sometimes, preceded ICP increase.

Conclusions: CEEG was necessary to diagnose and treat non-convulsive epileptic seizures and NCSE and as a guide to neuroprotection.

In any case neurophysiological monitoring stability was associated with cerebral damage evolution whereas a SEP deterioration has always revealed a neurological deterioration.

To our opinion, CEEG-SEP, as an index of cerebral function, might be a usefull tool integrated with multiparametric monitoring of comatose patients. The association of a pressure (ICP) and metabolic (SEP) index increases the monitoring sensibility to different pathogenetic mechanism of cerebral damage.

\section{B-2}

Consequence of the S100B protein levels for a prognosis of patients with severe head trauma injury

P. Lavicka1', M. Choc ${ }^{1}$, R. Pikner ${ }^{2}$, J. Mracek ${ }^{1}$, R. Bosman ${ }^{3}$, O. Topolcan ${ }^{2}$

${ }^{1}$ Department of Neurosurgery, ${ }^{2}$ Department of Nuclear Medicine,

${ }^{3}$ Department of Anaesthesiology and Intensive Care Medicine, Charles University Hospital, Pilsen, Czech Republic

Introduction: S100B protein is a small dimeric protein which belongs to a group of calcium binding proteins. It is present in high concentrations in astroglia cells. The level of this marker increases significantly with CNS damage. The aim of our research was to evaluate a consequence of a dynamic monitoring of the $\mathrm{S} 100 \mathrm{~B}$ protein levels for a prognosis of patients with severe head trauma injury.

Method: 60 patients with severe head trauma injury were prospectively monitored. All patients were admited to Emergency Department. The time period from injury to admission was shorter than 6 hours. All patients had the initial CT scan of the brain performed with a positive finding. We used these scoring protocols: at admission - GCS, APACHE II, at dismissal: GOS, Karnofsky Performance Scale. The S100B protein level was done by LIA Essay on fully automated immunoanalyser Liaison, Dia Sorin, Sweden. The S100B protein level measurement was done at admission, after 6, 12, 24 and 72 hours.

Results: The initial $\mathrm{S} 100 \mathrm{~B}$ protein level is significantly negative related to GOS and KPS $(p<0.0001)$. The initial S100B protein level prove better level of correlation with KPS and GOS then APACHE and GCS. The initial S100B protein level correlate tightly with KPS and GOS than following levels (time $6,12,24,72)$, but we found following correlations:

1. Rapid decrease of the levels in the first 72 hours to normal value is associated with a good prognosis.

2. Increasing or persistent high levels longer than 24 hours above normal value are associated with a poor outcome in GOS and KPS.

3. Repeated increase of S100B protein levels after previous decrease is associated with a higher incidence of the vegetative state and death.

4. The initial levels above 5 microg/L is always associated with death.

Conclusions: $\mathrm{S} 100 \mathrm{~B}$ protein is a good prognostic marker in comparison with APACHE and GCS, the course of a dynamic evaluation is by our opinion useful for more accurate determination of the prognosis.

\section{B-3}

Neurophysiological assessment in severe head trauma

A. Levati ${ }^{1}$, M.P. Moretti ${ }^{1}$, S. Meregalli ${ }^{2}$, C. Orsini ${ }^{1}$, S. Vesconi ${ }^{1}$, E. Facco ${ }^{3}$

${ }^{1}$ Neurointensive Care Unit and ${ }^{2}$ Neurophysiological Unit Niguarda H. Milan, ${ }^{3}$ Department of Pharmacology and Anesthesiology University of Padua, Italy

Introduction: The management of severe head trauma has progressively improved in the past years, but mortality and disability remain still high. Despite a prevailing agreement on Evoked Potentials and EEG effectiveness in the early prognosis of post-traumatic coma exists in the literature, the published data include conflicting results, discrepancies and contradictions, which make hard, or even impossible, any comparison between series.

The aim of our prospective study was to evaluate the sensitivity (Se) and specifity $(\mathrm{Sp})$ of somatosensory evoked potentials (SEPs) and EEG in the assessment of early prognosis of post-traumatic coma.

Methods: 29 consecutive patients $(21 \mathrm{M} / 8 \mathrm{~F}$; age range $15-66$ years, mean \pm SD $32 \pm 16$ ) with GCS of 8 or less were included from 1/10/2003 to $30 / 11 / 2004$. Exclusion criteria were: spinal cord injury, lesions of the brachial plexus, fractures of the temporal bone, haemotympanum, GCS $=3$ after clinical stabilization, and anoxia. Neurological examination, ICP and CPP were monitored in all patients throughout the clinical course. BAEPs and SEPs were recorded within $48 \mathrm{hs}$ from the injury, on the 5 th and 10 th hospital day. SEPs were graded as follows: $a=$ bilaterally normal; $b=$ monolateral increase of N13-N20 interval or absent N20; c = bilaterally increased 
N13-N20 interval; $d=$ bilaterally absent N20. EEG was graded as follows: $a=$ reactive to stimulus; $b=$ unreactive to stimulus or burst suppression. The Glasgow Outcome Score (GOS) was evaluated after 6 months from the trauma: good, moderate and severe disability were considered as favourable outcome (F.O.); persistent vegetative state and death were considered poor outcome (P.O.).

Results: 24 patients came out of coma (82.7\%) while the remaining 5 (17.3\%) died or remained vegetative.

SEPs was significantly related to the outcome at first $(P<0.05)$ and second test $(P<0.01)$; Se was $0.80,0.75,0.66$ at the first, second and third test, respectively, while $\mathrm{Sp}$ was $0.83,0.95,0.90$. The EEG was not significantly related to the outcome; however, Se was 1.0 throughout the study, while Sp was $0.42,0.33,0.61$ at the three tests.

Conclusion: Our result confirm the prognostic power of SEPs; being Sp higher than Se, they look to be better predictors of P.O. than F.O. EEG was not significantly related to the outcome due to a high rate of falsely pessimistic predictions; however, it showed a very high Se, thus excluding falsely optimistic errors. These data suggest that the combined use of EEG and SEPs may improve the assessment of severe head injury in comparison to the use of one investigation only.

Reference:

1 Classen J, Hansen HC. Early recovery after closed traumatic head injury: somatosensory evoked potentials and clinical findings. Crit Care Med 2001; 29: 494-502.

\section{B-4}

Is monitoring intracranial pressure in patients with malignant middle cerebral artery infarction useful?

M.A. Poca ${ }^{1}$, J. Sahuquillo', M. Riveiro², A. Rovira ${ }^{3}$, P. Delgado 4 , J. Álvarez-Sabín ${ }^{4}$

${ }^{1}$ Department of Neurosurgery and Neurotraumatology - Neurosurgery Research Unit, ${ }^{2}$ Neurotraumatology ICU, ${ }^{3}$ Institute of Diagnostic Imaging, Magnetic Resonace Unit and ${ }^{4}$ Neurovascular Unit and Neurology Department, Vall d'Hebron University Hospital, Barcelona, Spain

Introduction: Malignant middle cerebral artery infarction (MMCAI) is associated with a high mortality rate. Intracranial pressure (ICP) monitoring may be useful in the management of this entity. Some studies have suggested that neurological deterioration may be independent of intracranial hypertension. The aim of this study was to describe the findings of continuous ICP monitoring in patients with MMCAl, and to correlate these findings with pupillary abnormalities and midline shift.

Methods: Between January 2002 and May 2005, 21 patients with MMCA were treated in our unit, using a combined protocol of initial moderate hypothermia and hemicraniectomy. The latter was performed when patients presented a midline shift $\geqslant 5 \mathrm{~mm}$. Parenchymal ICP was monitored in all patients. In 13 patients, hourly ICP values and ICP pattern were evaluated and correlated with pupillary abnormalities (size and reactivity) and follow-up CT scans.

Results: Hemicraniectomy was performed in all 13 patients. The median time from initial ICP monitoring to surgery was 24 hours (interquartile range: $12-63)$. In seven of the 13 patients ICP was always $\leqslant 20 \mathrm{mmHg}$, but pupillary size ipsilateral to the infarction was increased in four

Conclusions: ICP monitoring is a highly sensitive indicator of neuroworsening, but has low specificity. Therefore, it cannot substitute close clinical and radiological follow-up in these patients. Pupillary abnormalities secondary to brain herniation may be present despite normal ICP values.

\section{B-5}

The misleading effect of cerebrovascular autoregulation variability on pressure-volume index assessment in clinical practice

A. Lavinio, F. Rasulo, E. De Peri, A. Candiani, N. Latronico Institute of Anesthesiology and Intensive Care Medicine, University of Brescia, Italy

Introduction: The pressure-volume index (PVI) is the best known parameter for assessment of volume-pressure relationship of the craniospinal space. It is defined as the volume of a bolus injection required to achieve a tenfold increase in intracranial pressure. The PVI has been previously criticized as being based on an "oversimplified" mathematical model. The aim of the present study was to verify if cerebrovascular autoregulation status can affect PVI assessment in clinical environment, as predicted by mathematical models [1] and observed in animal studies [2].

Methods: Observational, prospective study approved by local Ethics Committee. PVI and autoregulation status were both assessed every 48 hours in 23 critically ill mechanically ventilated comatose patients. Autoregulation was measured by means of transcranial Doppler ultrasonography and Transient Hyperemic Response Test (THRT) performed by trained medical doctors. PVIs were grouped by autoregulation efficacy and compared by repeated measures linear mixed model analysis.

Results: $78 \mathrm{PVI}$ and $78 \mathrm{THR}$ tests were performed in 23 patients. 4 patients underwent decompressive craniectomy and were separately analyzed. Average $\mathrm{PVI}$ in patients with excellent autoregulation (group A: THRR $>1.1$ ) was $20.0 \mathrm{ml}$ (SD10.2). Average PVI in the defective autoregulation group (group B: THRR < 1.1) was $31.6 \mathrm{ml}$ (SD18.8). Mean PVI difference was $11.6 \mathrm{ml}$ $(99 \%$ C.I. $=1.5 \mathrm{ml}$ to $21.7 \mathrm{ml}$; P $<0.01)$. ICP and CPP were not significantly different between group $A$ and group $B$. Similar results were obtained in the groups of patients who underwent decompressive craniectomy.

Conclusions: Cerebral autoregulation status affects PVI assessment independently from CPP and ICP. PVI test tends to measure a more compliant brain in patients with altered cerebrovascular autoregulation than in patients with intact cerebrovascular autoregulation. This implies that PVI cannot be considered an independent prognostic factor. Our results question that PVI accurately predict tolerance of the intracranial system to slow volume loads such as developing brain edema. Finally, our results suggest that the applicability of the Marmarou model should be critically reconsidered.

References:

1 Ursino M, Lodi CA, Rossi S, Stocchetti N. Estimation of the main factors affecting ICP dynamics by mathematical analysis of PVI tests. Acta Neurochir 1998; 71: 306-309.

2 Gray J, Rosner M. Pressure-volume index as a function of cerebral perfusion pressure. J Neurosurg 1987; 67: 377-380.

\section{B-6}

Cerebrovascular autoregulation changes in sepsis syndrome

F. Zubani, E. De Peri, F. Rasulo, A. Candiani, N. Latronico

Institute of Anesthesiology - Intensive Care, University of Brescia,

Spedali Civili, Italy

Introduction: More than $70 \%$ of patients with sepsis syndrome or septic shock present altered mental status manifested by decreasing attention, disorientation, agitation, obtundation and in worst cases, stupor and coma. This syndrome, known as "Septic Encephalopathy" (SE), usually occurs early in the course of sepsis and is associated with high mortality. Pathophysiology is unknown [1], but many clinical studies have shown that cerebral blood flow is reduced in patients with SE [2].

The current study was performed to evaluate cerebrovascular autoregulation during systemic inflammatory response syndrome (SIRS), sepsis syndrome, septic shock and multiple organ failure (MOF).

Methods: All patients with SE admitted from January 2004 to September 2004 were studied. Cerebral autoregulation was evaluated bilaterally in the MCA territory with the Transient Hyperemic Response Test (THRT).

Results: Twelve patients were included and 50 THRT were performed. Cerebral autoregulation was impaired in $64 \%$ of cases. The 2 conditions most frequently associated with abnormal cerebrovascular response were sepsis $(66.6 \%)$ and MOF $(80 \%)$. Patients with septic shock had the best autoregulatory response. In 6 measurements in patients with gram-negative infections cerebrovascular autoregulation was impaired in $100 \%$ of cases. In 12 measurements in patients with hypercapnia autoregulation was impaired in $83 \%$ of cases.

Conclusions: Cerebral autoregulation is frequently impaired in patients whit infections. Impaired autoregulation is associated with gram-negative infections and hypercapnia.

References:

1 Bolton CF. The neurological complications of sepsis. Ann Neurol 1993; 33: 94-100.

2 Bowton DL et al. Cerebral blood flow is reduced in patients with sepsis syndrome. Crit Care Med 1989; 17: 399-403.

\section{B-7}

Non-invasive monitoring of carbon dioxide reactivity: spatially resolved near infrared spectroscopy and transcranial Doppler

M. Tisdall ${ }^{1}$, I. Tachtsidis ${ }^{2}$, C.E. Elwell ${ }^{2}$, M. Smith ${ }^{1}$

${ }^{1}$ Department of Neuroanaesthesia and Neurocritical Care, The National Hospital for Neurology and Neurosurgery, ${ }^{2}$ Department of Medical Physics and Bioengineering, University College London

Introduction: Near infrared spectroscopy (NIRS) is a continuous noninvasive technique able to measure absolute changes in concentrations of oxy-haemoglobin $\left(\left[\mathrm{HbO}_{2}\right]\right)$ and deoxy-haemoglobin ( $\left.[\mathrm{HHb}]\right)$, and the absolute mixed arterial and venous oxygen saturation, termed tissue oxygenation index (TOI). TOI has been shown to have high sensitivity and specificity for changes in intracranial haemodynamics [1]. Changes in $[\mathrm{HbT}]\left(\left[\mathrm{HbT}=\left[\mathrm{HbO}_{2}\right]+[\mathrm{HHb}]\right)\right.$ reflect changes in cerebral blood volume. Transcranial Doppler ultrasonography (TCD) allows calculation of blood flow velocity in the basal cerebral arteries and provides a measure of scaled cerebral blood flow (CBF). Our previous work investigating carbon dioxide reactivity suggests that there may be a relationship between CBF and TOI. 
The aim of this study is to investigate the response of cranial NIRS and TCD variables to changes in end tidal carbon dioxide tension $\left(\mathrm{EtCO}_{2}\right)$.

Methods: Following institutional ethical approval and informed consent, 6 healthy volunteers were studied. A 2 channel NIRO 300 NIRS system (Hamamatsu Photonics $\mathrm{KK}$ ) recorded bilateral changes in $[\mathrm{HbT}]$ and absolute TOI. Blood flow velocity in the basal right middle cerebral artery (vMCA) was measured using TCD (Nicolet). $\mathrm{EtCO}_{2}$ (Novametrix) and inspired oxygen concentration $\left(\mathrm{FiO}_{2}\right)$ were recorded from a mouthpiece. Blood pressure (MBP) was measured non-invasively (Portapres ${ }^{\circledR}$ ) and arterial oxygen saturation $\left(\mathrm{SaO}_{2}\right)$ was monitored beat-to-beat. Data were collected during (i) 5 mins breathing room air; (ii) 5 mins hyperventilation to reduce $\mathrm{EtCO}_{2}$ by $1.5 \mathrm{kPa}$; (iii) 5 mins at baseline $\mathrm{EtCO}_{2}$; (iv) 5 mins breathing $6 \%$ inspired $\mathrm{CO}_{2}$ and (v) 5 mins at baseline $\mathrm{EtCO}_{2}$.

Results: Results are summarised as median and interquartile range for percentage change from baseline for vMCA and absolute changes from baseline for all other variables. Statistical analysis was performed using Wilcoxon signed ranks test and calculating 1 tailed exact significance. Average $\mathrm{HbT}$ and TOI were calculated from mean of bilateral data.

\begin{tabular}{|c|c|c|c|c|c|c|c|c|}
\hline & $\begin{array}{l}\Delta \mathrm{FiO}_{2} \\
(\%)\end{array}$ & $\begin{array}{l}\Delta \mathrm{SaO}_{2} \\
(\%)\end{array}$ & $\begin{array}{l}\Delta \mathrm{EtCO}_{2} \\
(\mathrm{kPa})\end{array}$ & $\begin{array}{l}\Delta \mathrm{HR} \\
\left(\min ^{-1}\right)\end{array}$ & $\begin{array}{l}\Delta \text { MBP } \\
(\mathrm{mmHg})\end{array}$ & $\begin{array}{l}\Delta \text { vMCA } \\
(\%)\end{array}$ & $\begin{array}{l}\Delta[\mathrm{HbT}] \\
\left(\mu \mathrm{moll}^{-1}\right)\end{array}$ & $\begin{array}{l}\Delta \text { TOI } \\
(\%)\end{array}$ \\
\hline Hyperventilation & 0.3 & $0.5^{\star}$ & $-1.7^{\star}$ & 2.3 & -1.6 & $-29.5^{\star}$ & $-1.4^{\star}$ & $-3.5^{\star}$ \\
\hline 25th percentile & $<0.1$ & 0.2 & -1.8 & -0.8 & -5.7 & -35.6 & -0.3 & -2.4 \\
\hline 75th percentile & 1.3 & 1.2 & -1.5 & 8.8 & 3.2 & -25.0 & -2.6 & -4.4 \\
\hline Hyperce & 1.1 & $0.7^{\star}$ & $1.7^{\star}$ & 2.3 & 3.9 & $18.1^{\star}$ & $3.1^{\star}$ & $5.1^{\star}$ \\
\hline 25th $\mathrm{p}$ & 0.1 & 0.4 & 1.1 & -0.8 & -1.3 & 2.5 & 2.4 & 4.4 \\
\hline 75th percentile & 1.4 & 1.1 & 2.0 & 8.8 & 7.8 & 33.4 & 3.6 & 6.0 \\
\hline
\end{tabular}

* indicates $p<0.05$ compared to baseline.

Conclusions: Changes in arterial $\mathrm{CO}_{2}$ partial pressure cause changes in arteriolar diameter and cerebral blood flow and these changes can be detected using NIRS. The TCD data show corresponding changes in VMCA The induced variations in $\mathrm{CO}_{2}$ cause changes in $[\mathrm{HbT}]$ which reflect alterations in cerebral blood volume. TOI also responds to changes in $\mathrm{EtCO}_{2}$ and this may be due to alteration in arterial: venous volume ratio and/or reduced oxygen extraction secondary to increased cerebral blood flow. Our findings have implications for the analysis of NIRS data from brain injured patients during $\mathrm{CO}_{2}$ reactivity testing.

Reference:

1 Al-Rawi et al. Evaluation of a near-infrared spectrometer (NIRO 300) for the detection of intracranial oxygenation changes in the human head. Stroke 2001; 32(11) $2492-4628$

\section{B-8}

Continuous PtO2 and ICP measurements in awake patients with suspected normal pressure hydrocephalus (NPH) conclusions for the debate on critical cerebral PtO2

\section{thresholds in the ICU setting}

H. Wessling, C.L. Simosono, M. Escosa, P. de las Heras

Unit of Neurosurgery, Hospital Universitari De Tarragona Joan XXIII,

Tarragona, Spain

Introduction: During the last decade, continuous invasive $\mathrm{PtO} 2$ recording has become a widely accepted and well established mean of monitoring for patients with acute traumatic or spontaneous cerebral lesions. However critical thresholds of ischemia and normal values are still a matter of debate. This retrospective study tries to draw conclusions for that debate from the interpretation of $\mathrm{PtO} 2$ values that have been recorded in awake patients with clinically and radiologically suspected normal pressure hydrocephalus.

Methods: Data from 10 patients with suspected NPH, who had a combined ICP and PtO2 probe (Ventrix/Licox) inserted in their right frontal lobe (local anesthesia) are analysed for mean $\mathrm{PtO} 2$ levels during day and night. Correlation coefficients between ICP and PtO2 are calculated. The results are interpreted considering age, gender, medical history, mean arterial pressure values, peripherical oxygen saturation. The differences between the group of patients who later had a shunt implanted and the group of patients who where not considered surgical are underlined. The registered values will be compared with normal values and critical threshold values for brain ischemia that where derived from the pertinent literature.

Results:

1. In the surgical group we found a mean $\mathrm{PtO} 2$ level of 15.8 during the day and 19.38 during the night. Mean Values ranged from $8.1 \mathrm{mmHg}$ to $20.4 \mathrm{mmHg}$ during the day and from $8.2 \mathrm{mmHg}$ to $26.6 \mathrm{mmHg}$ during the night.

2. In the non-surgical group we found a mean PtO2 level of $19.16 \mathrm{mmHg}$ during the day and $18.17 \mathrm{mmHg}$ during the night. Mean values ranged from $17.1 \mathrm{mmHg}$ to $21.0 \mathrm{mmHg}$ during the day and from $7.9 \mathrm{mmHg}$ to $22.4 \mathrm{mmHg}$ during the night.

3. In spite of episodes of extremely low PtO2 levels in 2 patients, no brain ischemia was observed.
Conclusions: Our results indicate that threshold values and normal values that can be found in the pertinent literature do not necessarily reflect actual cerebral oxygen metabolism in individual patients. These values may need to be defined on an individual base considering parameters which are unrelated to the acute brain lesion.

Ethics committee approval: The study has been presented to and approved by the local ethics committee.

\section{B-9}

Association between impaired cerebral autoregulation and outcome in severe pediatric traumatic brain injury M.S. Vavilala' ${ }^{1}$, S. Muangman'2 ${ }^{2}$ P. Suz ${ }^{3}$, M.S. Kincaid ${ }^{4}$, A.M. Lam ${ }^{5}$

${ }^{1-5}$ Departments of Anesthesiology, ${ }^{1}$ Pediatrics and ${ }^{1,5}$ Neurological Surgery, University of Washington, Seattle, WA

Introduction: Impaired cerebral autoregulation following TBI may be associated with secondary brain injury and poor outcome. The purpose of this study was to examine: (1) the incidence of impaired cerebral autoregulation and (2) the relationship between cerebral autoregulation and outcome following severe pediatric TBI.

Methods: Children $<18$ years with diffuse TBI and Glasgow Coma Scale (GCS) score $<9$ were prospectively enrolled at Harborview Medical Center's (level 1 Pediatric Trauma Center) Pediatric Intensive Care Unit, Seattle,WA between May 2002-March 2005

Transcranial Doppler (TCD) ultrasonography was used to measure bilateral middle cerebral artery flow velocities (Vmca). Static autoregulation testing was performed within 48 hours of admission by administering intravenous phenylephrine to increase cerebral perfusion pressure (CPP) to the greater of either $20 \%$ above baseline or age dependent thresholds $(80 \mathrm{mmHg}$ for age $<9$ years or $90 \mathrm{mmHg}$ for age 9-16 years). Simultaneous changes in CPP and Vmca were continuously measured. Cerebral autoregulation was quantified using the Autoregulatory Index (ARI; \% change in estimated cerebrovascular resistance in response to the \% change in CPP).

Autoregulatory capacity was dichotomized to reflect impaired $(A R I<0.4)$ and intact $(A R I \geqslant 0.4)$ cerebral autoregulation. Glasgow outcome score (GOS) < 4 represented poor outcome and was tested at 3, 6 and 12 months. Data are given as mean \pm SD. Fishers' Exact test and Student's $T$ test were used. $\mathrm{p}<0.05$ reflected significance.

Results: 28 children (20M and $8 F) 1.3-17$ years $(10 \pm 5)$ were enrolled. ARI was $<0.4$ in $12 / 28(43 \%)$ patients. GOS was $<4$ in $12 / 28(43 \%)$ at 3 months vs. $10 / 27(37 \%)$ at 6 months and $7 / 23(30 \%)$ at 12 months. ARI $<0.4$ was associated with GOS $<4$ at 6 and 12 months (Tables 1, 2).

Conclusions: A significant number of children had impaired cerebral autoregulation during the first 48 hours after severe TBI. Although outcome improved following discharge, early impaired cerebral autoregulation was associated with poor outcome at 6 and 12 months.

Table 1. ARI and 6 month GOS; $(\mathrm{p}=0.007)$.
\begin{tabular}{llc}
\hline $\mathbf{N}=\mathbf{2 7}$ & $\mathbf{G O S}<\mathbf{4}$ & $\mathbf{G O S} \geqslant \mathbf{4}$ \\
\hline $\mathrm{ARI}<0.4$ & 8 & 4 \\
$\mathrm{ARI} \leqslant 0.4$ & 2 & 13 \\
\hline
\end{tabular}

Table 2. ARI and 12 month GOS; $(p=0.02)$.

\begin{tabular}{llc}
\hline $\mathbf{N}=\mathbf{2 3}$ & $\mathbf{G O S}<\mathbf{4}$ & $\mathbf{G O S} \geqslant \mathbf{4}$ \\
\hline $\mathrm{ARI}<0.4$ & 6 & 4 \\
$\mathrm{ARI} \geqslant 0.4$ & 1 & 12 \\
\hline
\end{tabular}

Reference:

1 Vavilala, MS, Lee LA, Newell DW, Lam AM. Cerebral autoregulation in children with traumatic brain injury. Pediatr Crit Care Med 2004; 5: 257-263.

\section{B-10}

\section{Cerebral blood flow autoregulation during experimental S. pneumoniae meningitis}

M. Pedersen ${ }^{1,3}$, C.T. Brandt ${ }^{3}$, G.M. Knudsen ${ }^{2}$, P. Skinhøj ${ }^{1}$, K. Møller ${ }^{1}$

${ }^{1}$ Department of Infectious Diseases, ${ }^{2}$ Neurobiology Research Unit, Rigshospitalet, Copenhagen University Hospital and ${ }^{3}$ Division of Microbiology, Statens Serum Institut, Copenhagen, Denmark

Background: Streptococcus pneumoniae meningitis remains a life-threatening disease with a mortality of $14-25 \%$ and severe neurological sequelae in $30-50 \%$ of the survivors. In healthy man, a constant blood supply to the brain is secured during changes in cerebral perfusion pressure (CPP) by cerebral blood flow (CBF) autoregulation. The objective of this study was to study CBF autoregulation in a rat model of meningitis. 
Methods: The study was based on a model of bacterial meningitis in male Harlan Wistar rats using an intracisternal injection of bacteria (S. pneumoniae type $3,1.7 \times 10^{7} \pm 1.09 \times 10^{7}$ (mean $\pm \mathrm{SD}$ ) colony forming units (CFU) per $\mathrm{ml}$ ) or a corresponding volume of saline. Twenty-four hours after inoculation, animals were anaesthetized using isoflurane, intubated, mechanically ventilated and cannulated for invasive measurement of mean arterial blood pressure (MAP) and infusion of fluids and medicine. Intracranial pressure (ICP) was measured by intracranial catheterization and CPP was calculated as (MAP-ICP). CBF was measured with Laser-Doppler flowmetry (during norepinephrine infusion to increase the CPP, as well as) during exsanguination to reduce the CPP. Predefined criteria were used to assess whether CBF autoregulation was present or absent.

Results: Clinical signs of meningitis were observed in all animals $(N=20)$ inoculated with $S$. pneumoniae and in none of the saline-inoculated animals $(\mathrm{N}=20)$. The bacterial concentration in the cerebrospinal fluid was $6.6 \times 10^{7} \pm 4.2 \times 10^{7} \mathrm{CFU}$ per $\mathrm{ml}$. The intracranial pressure in meningitis animals was significantly higher than in control animals $(14 \pm 4$ vs. $3 \pm 1 \mathrm{mmHg})(\mathrm{P}<0.01)$.

During normocapnia $\left(\mathrm{P}_{\mathrm{a}} \mathrm{CO}_{2}=5.1 \pm 1.6 \mathrm{kPa}\right.$, mean $\left.\pm \mathrm{SD}\right)$, a lower limit of autoregulation was present in 8 of 10 control animals and 1 of 10 animals with meningitis (Fisher's exact test, $\mathrm{P}<0.01$ ). The upper limit was present in 5 of 5 controls and in none of 5 meningitis rats. During acute hypocapnia $\left(\mathrm{P}_{\mathrm{a}} \mathrm{CO}_{2}=3.7 \pm 0.2 \mathrm{kPa}\right)$, the lower limit of autoregulation was present in 10 of 10 control animals and in 6 of $10 \mathrm{~S}$. pneumoniae-inoculated animals (Fisher's exact test for comparison with normocapnia, $\mathrm{P}<0.05)$. The upper limit was not studied during hypocapnia. The intracranial pressure in hypocapnic meningitis rats did not differ significantly from the normocapnic meningitis rats $(13 \pm 5$ vs. $20 \pm 4 \mathrm{mmHg})$.

Conclusion: CBF autoregulation is abolished in rats during early S. pneumoniae meningitis. Acute hypocapnia restores the autoregulation in some animals, in the absence of significant changes in the intracranial pressure. This may be of clinical importance, as it inhibits fluctuations in perfusion pressure.

\section{Group C: Clinical Interventions}

\section{C-1}

Efficiency of erythropoietin to reduce blood transfusion during craniosynostosis surgical repair: a preliminary study

S. Grousson ${ }^{1}$, J. Convert ${ }^{1}$, T. Lieutaud ${ }^{1}$, C. Lak ${ }^{1}$, C. Mottolese ${ }^{2}$,

A. Szathmari' ${ }^{2}$, F. Artru ${ }^{1}$

${ }^{1}$ Department of Neuroanesthesia and ${ }^{2}$ Pediatric Neurosurgical Unit, Neurological Hospital, Lyon

Introduction: Perioperative homologous blood transfusion $(\mathrm{HT})$ is required in most cases of patients undergoing extensive surgical procedures due to craniofacial deformities. Several studies have highlighted the reduction of the amount of HT with prior administration of exogenous erythropoietin [1,2]. Methods: A retrospective review of consecutive pediatric patients with major surgical reconstruction for craniosynostosis was performed during a 3-years period: for local medical reasons, recombinant human erythropoietin ( $\mathrm{rHuEpo}$ ) pretreatment with iron supplementation were given in 2001 and 2003 (Erythropoietin group); however, this blood sparing technique was not used in 2002 (Control group). All children were operated by the same neurosurgeon. Demographic characteristics as age, weight and surgical time were collected for both populations. Criteria for intra and postoperative HT were established by the department of neuroanesthesiology. Comparisons between treatment and control groups data (presented as means \pm standard deviation) were made by Chi square or one-way analysis of variance (ANOVA) with statistical significance at $p$ of 0.05 or less.

Results: From the 76 infants and small children eligible for the study, 39 were assigned to receive erythropoietin treatment $(\mathrm{rHuEpo}+)$. A total of 50 patients required HT. Of these, 16 in the rHuEpo+ group did receive blood products and 34 in the rHuEpo- control group did not $(p<0.001)$. The erythropoietin-treated subjects were younger (13 \pm 8 m.o.) than the control $(25 \pm 29$ m.o.) with $p$ value $<0.01$. Duration of surgery was comparable for both groups ( $221 \pm 54$ and $229 \pm 54 \mathrm{~min}$. respectively).

Conclusions: In this study, rHuEpo administration to children undergoing craniosynostosis reconstruction was associated with a decrease in transfusion requirements from 92 to $41 \%$ (risk ratio $=0.55$ ). A large randomized controlled trial with double blind design is mandatory in this setting.

References:

1 Helfaer MA et al. Increased hematocrit and decreased transfusion requirements in children given erythropoietin before undergoing craniofacial surgery. $J$ Neurosurg 1998; 88: 704-708.

2 Fearon JA, Weinthal J. The use of recombinant erythropoietin in the reduction of blood transfusion rates in craniosynostosis repair in infants and children. Plastr Reconstr Surg 2002; 109: 2190-2196.

\section{C-2}

Decompressive craniectomy in traumatic brain injury: the randomised multi-centre RESCUEicp study www.rescueicp.com

P.J. Hutchinson, E. Corteen, M. Czosnyka, A.D. Mendelow, D.K. Menon, P. Mitchell, G. Murray, J.D. Pickard, E. Rickels, J. Sahuquillo, F. Servadei, G.M. Teasdale, I. Timofeev, A. Unterberg, P.J. Kirkpatrick

University of Cambridge and European Brain Injury Consortium

Introduction: One of the fundamental pathophysiological processes following traumatic brain injury is the development and propagation of an escalating cycle of brain swelling, increase in intra-cranial pressure (ICP), reduction in blood and oxygen supply, energy failure and cell death. While in most patients ICP can be controlled using fundamental medical measures (ventilation, sedation, inotropes, mannitol etc) in others raised ICP becomes refractory. These patients may benefit from "decompressive craniectomy". There are several studies in the literature investigating the role of decompressive craniectomy in traumatic brain injury. These demonstrate a wide range of outcomes with no clear consensus regarding the indications for and the results following the operation. We are therefore proposing a multicentre randomised trial (Randomised Evaluation of Surgery with Craniectomy for Uncontrollable Elevation of ICP - the RESCUEicp study) coordinated by the University of Cambridge Department of Neurosciences in collaboration with the European Brain Injury Consortium. The objective of the study is to investigate whether decompressive craniectomy results in improvement in outcome and surrogate endpoints compared to contemporary optimal medical management.

Methods: The trial aims to recruit from centres experienced in the intensive care management of head injury. The target study group is ventilated ICPmonitored patients with refractory intracranial hypertension. The inclusion criteria are traumatic brain injury, age 10-65 years with an abnormal CT scan requiring ICP monitoring with raised ICP $(>25 \mathrm{mmHg})$ refractory to initial medical treatment measures. The exclusion criteria are bilateral fixed and dilated pupils on admission, bleeding diathesis and devastating injury not expected to survive for 24 hours. The two randomised arms are continuation of optimal medical management (including barbiturates) versus surgery (decompressive craniectomy). Outcome is assessed using the Extended Glasgow Outcome Score at six months as the primary measure with secondary measures including the SF-36 quality of life questionnaire, control of ICP, time in intensive care and time to discharge from the neurosurgical unit. The study aims to recruit patients from approximately 40 centres worldwide. The RESCUEicp study has now commenced. This presentation will outline the background, protocol and progress to date.

\section{C-3}

Pentobarbital versus thiopental in patients with refractory intracranial hypertension. Preliminary results of a pilot study comparing their efficacy in severe traumatic brain injury

J.P. Barcena ${ }^{1}$, J.A.L. Pou' ${ }^{1}$, J. Homar ${ }^{1}$, J.M. Abadal', J.M. Raurich ${ }^{1}$, J.I. Juve ${ }^{1}$, B. Castanayer ${ }^{2}$, J. Sahuquillo ${ }^{3}$

${ }^{1}$ Intensive Care Department, Hospital Universitario Son Dureta, Palma de Mallorca, ${ }^{2}$ Servicio de Análisis Clínicos, Hospital Son Dureta, ${ }^{3}$ Department of Neurosurgery, Vall d'Hebrón University Hospital, Barcelona, Spain

Introduction: Barbiturate coma has been used for decades in patients with severe traumatic brain injury (TBI), and its use is supported by The Brain Trauma Foundation (BTF) guidelines to control high intracranial pressure (ICP) [1]. Both thiopental and pentobarbital have been used. Although several experimental studies have shown that both treatments might not be equally effective [2], to date no clinical study has been published comparing the efficacy of pentobarbital and thiopental. The goal of this study was to prove or refute the hypothesis that thiopental is a better option than pentobarbital to 
control raised ICP refractory to first tier therapeutic measures in patients with severe TBI.

Methods: Prospective, single-blind randomized study to compare the efficacy of pentobarbital and thiopental in controlling refractory high ICP in patients with a severe TBI (postresuscitation Glasgow Coma Scale GCS $=8$ points). All patients were admitted to the Intensive Care Unit and managed according to the recommendations of the BTF guidelines. Patients were randomized when ICP was above $20 \mathrm{mmHg}$ despite first tier therapeutic measured. Patients assigned to the pentobarbital arm received a loading dose of $10 \mathrm{mg} / \mathrm{kg}$ over 30 minutes plus $5 \mathrm{mg} / \mathrm{kg} /$ hour for 3 hours followed by a maintenance dose of $1 \mathrm{mg} / \mathrm{kg} / \mathrm{hour}$. Patients assigned to the thiopenta arm received an initial bolus of $5 \mathrm{mg} / \mathrm{kg}$ followed by a maintenance dose of $3 \mathrm{mg} / \mathrm{kg} /$ hour. Treatment was considered successful when ICP fell below $20 \mathrm{mmHg}$ and remained below that level for at least 48 hours. Treatment failure was considered when ICP remained uncontrollable.

Results: We present the results of the first 29 patients included. Fifteen received pentobarbital and fourteen thiopental. There were no statistically significant differences between the two groups in age, sex, GCS at admission, initial CT scan and severity of associated injuries. Thiopental controlled raised ICP in five patients $(36 \%)$ and pentobarbital in only two patients $(13 \%)$ $(P=0.16)$. Seven patients in the thiopental group and eleven in the pentobarbital group died $(P=0.19)$. There were no statistically significant differences between the two groups in hypotension $(P=0.96)$ or infectious complications. Conclusions: These preliminary results suggest that the efficacy of thiopental is greater that the pentobarbital in controlling high refractory ICP. These results support previous experimental findings showing that the two treatments are not equally and justify a larger study to confirm or disconfirm these results.

\section{References:}

1 The Brain Trauma Foundation. Use of barbiturates in the control of intracranial hypertension. J Neurotrauma 2000; 17: 527-537.

2 Drummond JC. Cerebral protection: are all barbiturates created equal? Anesthesiology 1996; 85: 1504-1505.

\section{C-4}

Decompressive craniectomy following traumatic brain injury leads to reduction in intracranial pressure and improves cerebral autoregulation

I. Timofeev ${ }^{1}$, M. Czosnyka ${ }^{1}$, P.J. Kirkpatrick ${ }^{1}$, J. Nortje², D.K. Menon ${ }^{2}$, A.K. Gupta ${ }^{2}$, J.D. Pickard ${ }^{1}$, P.J. Hutchinson ${ }^{1}$

${ }^{1}$ Department of Neurosurgery, Addenbrooke's Hospital, Cambridge, ${ }^{2}$ Division of Anaesthesia, University of Cambridge, Cambridge

Introduction: Intracranial hypertension is common after severe traumatic brain injury and management aims to reduce high intracranial pressure (ICP) and to maintain an adequate cerebral perfusion pressure (CPP). Decompressive craniectomy (DC) is often performed when other measures of ICP control are exhausted. Although there is evidence that $\mathrm{DC}$ reduces ICP, the duration of this effect is unclear. The purpose of this study was to evaluate the effect of DC on ICP both within and beyond the initial post-operative 24 hours. The effect of surgery on cerebral autoregulation and pressure-volume compensatory reserve, represented by ICP waveform derived indices [1] was also assessed.

Methods: Medical records and intensive care charts of 23 patients who underwent DC with recording of pre and postoperative ICP values and other physiological parameters were analysed. Of these, 13 had continuous digital ICP recording with online waveform analysis (using ICM+software developed in house). Statistical analysis was performed with SPSS 13.0 for Windows. Tests appropriate to sample distribution were used and $p=0.05$ was considered significant.

Results: In all patients a progressive increase in ICP was observed preoperatively. The initial ICP(mean of means \pm SD $\mathrm{mmHg}$ ) was $18.5( \pm 6)$, increasing to $24( \pm 5)$ for the 24 hours preceding the operation. Mean ICP during the last 3 pre-operative hours was $30( \pm 4.7)$, despite maximum medical therapy. In the initial post-operative period (first 3 hours) ICP reduced to $13( \pm 4.7)$. ICP during day 1,2 , and 3 was $15( \pm 8.8), 15( \pm 7.2)$ and $17( \pm 7)$ with no statistically significant difference between these values. Mean ICP during the whole post-operative period of $14.5( \pm 6.9)$ and was significantly lower than pre-operative ICP $(p<0.005)$.

Decompressive craniectomy significantly reduced the pulse amplitude of ICP and amplitude of slow waves, and led to improvement in pressurevolume compensatory reserve (RAP). Mean CPP levels were above $70 \mathrm{mmHg}$ in pre- and post-operative periods, but required a significantly higher mean arterial pressure (MAP) pre-operatively.

Conclusion: The results of this study suggest that DC leads to a sustained reduction in ICP and improvement in cerebral autoregulation. Adequate CPP levels can be achieved at lower MAP levels. This reduces the load on the cardiovascular system and risks associated with aggressive CPP augmentation. Prospective, larger scale studies are required to further assess the effect of decompressive craniectomy on other physiological parameters and clinical outcome.

Reference:

1 Czosnyka M, Pickard JD. Monitoring and interpretation of intracranial pressure. J Neurol Neurosurg Psychiatry 2004; 75: 813-821.

\section{C-5}

\section{Context-sensitive corticosteroid use in head injury}

J.G. Outtrim ${ }^{1}$, F. Bernard ${ }^{1}$, D.K. Menon ${ }^{1}$, B.F. Matta ${ }^{2}$

${ }^{1}$ Division of Anaesthesia, University of Cambridge, Cambridge, ${ }^{2}$ Department of Anaesthesia, Addenbrooke's Hospital, Cambridge

The CRASH Trial [1,2] has raised concerns regarding the use of high-dose steroids in head injury, both in the scientific and lay press [3-5]. However these results cannot be extrapolated to other settings which are common co-morbidities in head injury. The Surviving Sepsis Campaign recommends that vasopressor dependent critically ill patients may benefit from steroids [6]. Further, adrenal insufficiency is common in septic shock and physiological replacement of hydrocortisone improves outcome [7].

We retrospectively analysed the records of 113 patients (mean age 35 years, $79 \%$ male, $70 \%$ severe head injury) who had an adrenal stimulation test performed within 10 days of admission using Tetracosactide (Synacthen ${ }^{\circledR}$ ). We defined adrenal insufficiency as baseline cortisol level below $414 \mathrm{mmol} / \mathrm{L}$ and failure to rise by $250 \mathrm{mmol} / \mathrm{L}$ after Synacthen ${ }^{\circledR}$ administration. All patients who showed adrenal insufficiency, or responded clinically to a trial of steroid therapy, received $200-400 \mathrm{mg}$ hydrocortisone per day while on vasopressor therapy. Patient outcome was defined using the 6-month Glasgow Outcome Score.

Adrenal insufficiency according to baseline cortisol levels was identified in $78 \%$ of patients; of these $60 \%$ received steroids, of which $57 \%$ were steroid responsive. Steroid responsive patients had a better outcome compared to non-steroid responsive patients (59\% versus 32\%). Injury and/or illness severity indices did not predict adrenal insufficiency.

Despite the results of the CRASH Trial, steroid use according to sepsis guidelines may provide benefit in head injury. The dose and clinical context are important in defining benefit from corticosteroids in head injury.

\section{References:}

1 Roberts I et al. Lancet 2004; 364: 1321

2 CRASH Trial Collaborators Lancet 2005, In Press.

3 Meikle J. The Guardian 8 October 2004; p. 12.

4 Derbyshire D. Daily Telegraph 8 October 2004; p. 31.

5 Sauerland S et al. Lancet 2004; 364: 1291.

6 http://www.sccm.org/professional_resources/

7 Annane D. Crit Care Med 2001; 29(S7): S117-S120.

\section{C-6}

Effects of acute treatment with Pravastatin on cerebral vasospasm, autoregulation, and delayed ischemic deficits after aneurysmal subarachnoid hemorrhage: a phase II randomised placebo-controlled trial

M.Y. Tseng, M. Czosnyka, H. Richards, J.D. Pickard, P.J. Kirkpatrick

Department of Neurosurgery, Addenbrooke's Hospital, University of Cambridge, Cambridge, United Kingdom

Introduction: Statins may improve cerebral vasomotor reactivity through cholesterol dependent and independent mechanisms. We assessed the hypothesis that acute Pravastatin treatment could improve cerebrovascular autoregulation and reduce vasospasm-related complications after aneurysmal subarachnoid hemorrhage (aSAH).

Methods: Approval was granted from the Local Research Ethics Committee (LREC No. 03/353) and the Medicines and Healthcare Products Regulatory Agency (MHRA No. MF/8000/13164). During 2004, 80 aSAH patients (age 18 to 84 years) within 72 hours from the ictus were randomised equally to receive either oral Pravastatin $(40 \mathrm{mg})$ or placebo daily for up to 14 days. Primary endpoints were the incidence, duration, and severity of cerebral vasospasm, and duration of impaired autoregulation estimated from transcranial Doppler ultrasonography. Secondary endpoints were the incidence of vasospasm-related delayed ischemic deficits (DID) and disability at discharge.

Results: Pre-randomisation characteristics were balanced between the two groups. No treatment-related complication was observed. The incidences of vasospasm and severe vasospasm were reduced by $32 \%(p=0.006)$ and $42 \%(p=0.044)$ respectively, and the duration of severe vasospasm was shortened by 0.8 day $(p=0.068)$ in the Pravastatin group. These measurements were maximal on the ipsilateral side of ruptured aneurysms. The duration 
of impaired autoregulation was shortened bilaterally $(p=0.01)$, and the incidence of vasospasm-related DID and mortality were decreased by $83 \%$ $(p<0.001)$ and $75 \%(p=0.037)$ respectively in the Pravastatin group. Conclusions: Acute treatment with Pravastatin after aSAH is safe and ameliorates cerebral vasospasm, improves cerebral autoregulation and reduces vasospasm-related DID. Unfavorable outcome at discharge were reduced primarily due to a reduction in overall mortality. This is the first demonstration of clinical benefits with immediate statin therapy for an acute cerebrovascular disorder.

\section{C-7}

\section{Empirical vs metabolic monitoring based nutritional support} in patients with hemorrhagic stroke

N.S. Gadgieva, I.N. Leiderman, A.A. Belkin

Clinical Institute of the Brain of the Urals Department of the Russian Academy of Medical Sciences, Yekaterinburg, Russia

Introduction: Severe protein-energy malnutrition (PEM) is one of the most important reasons of infectious complications in patients with stroke. The aim of our study was to compare the influence of empirical and metabolic monitoring based nutritional support on the incidence of some complications and clinical outcome in stroke patients.

Methods: Prospective comparative clinical trial of empirical and metabolic monitoring based nutritional support efficiency in patients with hemorrhagic stroke was carried out on the basis of Clinical Institute of the Brain of the Urals department of the Russian Academy of Medical Sciences (Yekaterinburg, Russia). This research was approved by Ethical Committee of Clinical Institute of Brain. Patients older than 18 years with severe hemorrhagic stroke without diabetes mellitus on mechanical ventilation more than 72 hours were included into our research. The presence of hemorrhagic stroke was confirmed by computer tomography or the magnetic-resonance tomography in all patients. In the group of empirical nutritional support $(n=36)$ the program of tube feeding and parenteral nutrition consisted of $80-100 \mathrm{~g}$ of protein and 2200-2500 kkal/day in all patients. In the group of metabolic monitoring based nutritional support $(n=54)$ daily protein and energy requirements were determined by metabolic monitoring procedure measurements: oxygen consumption, carbon dioxide excretion, resting energy expenditure (REE) calculations, urea nitrogen excretion and nitrogen balance calculations.

Results: The incidence of ventilator-associated pneumonia (VAP) was significantly lower in the group of metabolic monitoring based nutritional support: $3(54)-6 \%$ vs $10(36)-28 \%$ in empirical group $(p<0.01)$. The rate of skin lesions was also decreased in metabolic monitoring group: $6(54)-11 \%$ vs $9(36)-25 \%(p<0.05)$. There was no difference in mortality $23(54)-43 \%$ vs $17(36)-47 \%(p>0.05)$.

Conclusion: In patients with severe hemorrhagic stroke the metabolic monitoring based nutritional support may be useful for decreasing the incidence of VAP and skin lesions.

\section{C-8}

The effect of decompressive craniectomy on ICP in patients with traumatic brain injuries

\section{T. Skoglund, C. Eriksson-Ritzén, B. Rydenhag}

\section{Department of Neurosurgery, Sahlgrenska University Hospital, Göteborg,} Sweden

Introduction: In patients with traumatic brain injury (TBI) intracranial hypertension secondary to cerebral oedema is a major problem. We use decompressive craniectomy as a last tier treatment in patients who develop uncontrollable intracranial hypertension, despite maximal conventional surgery and medical treatment (including barbiturate sedation). In the present study we wanted to investigate the effect of craniectomy on ICP (intracranial pressure).

Methods: Among the about 150 patients with severe TBI treated at our neurointensive care unit during the period from 1997-2002, nineteen patients were treated with decompressive craniectomy. Information about the ICP before and after the decompressive cranictomy was used. The approximate area of the removed bone flap was calculated using the postoperative CT scans. In each image the length of the removed bone was measured. Using these measurements together with the slice thickness of the CT-scans, the total area of the removed bone flap was calculated and expressed in $\mathrm{mm}^{2}$.

Results: All patients were young (mean $22 \pm 11$ years, range $7-46$ ) and $68 \%$ were male. The mean ICP was reduced from $31.2 \pm 5.4$ before to $12.7 \pm 9.6 \mathrm{mmHg}$ immediately after the craniectomy, twenty-four hours after the craniectomy the mean ICP was $13.9 \pm 9.4 \mathrm{mmHg}$. Student's t-test revealed a statistically significant decrease both when comparing the preoperative values to the values immediately postoperative as well as to the values after twenty-four hours $(p<0.01)$.

For the analysis, the patients were divided into two groups - one with a large craniectomy (here defined as an area $>7.000 \mathrm{~mm}^{2}$ ) and one with a smaller $\left(<7.000 \mathrm{~mm}^{2}\right)$. The ICP of the patients with a large craniectomy was reduced by $77 \%( \pm 14)$ while the ICP of the patients with a small craniectomy was reduced by $44 \%( \pm 26)$. This difference was also found to be statistically significant $(\mathrm{p}<0.05)$.

Conclusion: A significant reduction in the ICP was found when comparing preoperative ICP with values directly as well as after 24 hours after the craniectomy. A large craniectomy led to a significantly larger decrease in ICP.

\section{C-9}

\section{Refractory posttraumatic intracranial hypertension:} treatment with an external lumbar drainage

J. Homar, J.M. Abadal, J.A. Llompart, J. Perez, J. Ibáñez

Intensive Care Unit, Hospital Universitario Son Dureta, Palma, Spain

Introduction: Intracranial hypertension $(\mathrm{ICH})$ is a frequent complication of severe Traumatic Brain Injury (TBI) appearing in $50-75 \%$ of the cases. The treatment of these patients is defined by the Brain Trauma Foundation Guidelines. Patients refractory to second line therapies show a mortality of $84-100 \%$. The aim of this study was to assess the external lumbar drainage (ELD) as a treatment for patients with $\mathrm{TBI}$ and $\mathrm{ICH}$ refractory to second line therapies.

Methods: The study was set in an Intensive Care Unit (ICU) of a third level University Hospital. We placed an ELD (Portex Limited, Hythe, Kent, CT21 $6 \mathrm{LJ}, \mathrm{UK}$ ) for continous drainage of the cerebrospinal fluid (CSF) in patients with posttraumatic $\mathrm{ICH}$ refractory to medical and surgical treatments (according to a standardized protocol). The ELD was placed through a 18gauge curved-tip needle into the subarachnoid space at L3-L4 level. A cranial computed tomography (CCT) scan performed in the previous 24 hour confirmed discernible basal cisterns, absence of any surgical mass and a midline shift minor than $10 \mathrm{~mm}$ [1] in all the cases. Informed consent was obtained from patient's relatives.

Results: We studied seventeen patients with a mean age of 32.6 (range 17-69) years. Most of them were males (76\%). The mean admission Glasgow Coma Scale (GCS) was 9(range 4-15). None of the patients had a ventriculostomy or a descompressive craniectomy. The mean intracranial pressure (ICP) before ELD was 30.9(range 21-51) $\mathrm{mmHg}$ and after ELD was 14.1(range 3-23) $\mathrm{mmHg}$. ICP was reduced in all patients allowing us to reduce and stop the second line $\mathrm{ICH}$ treatment (barbiturate coma and/or moderate hypothermia). In 4 patients, the ICP rebounded after 5 days of ELD use. These patients died in the ICU. The most common complication was ELD obstruction (5 cases). No positive cultures of CSF or catheter's distal end were obtained. We removed the drainage when ICP was controlled only with first tier measures and there were no new features in a repeated CCT scan. The average duration of the ELD was 7 days (range 4-15). The neurological outcome was evaluated by Glasgow Outcome Scale [2] (GOS) at ICU discharge and after 6 months of the TBI. All the patients who survived at ICU discharge (76\%) presented a good functional outcome after 6 months (GOS 1 and 2).

Conclusions: The external lumbar drainage is a therapeutic option in selected cases of refractory posttraumatic intracranial hypertension. The neurological outcome of these patients 6 months after the traumatic brain injury was dramatically improved. Few complications were associated with the ELD. References:

1 Münch EC, Bahuf C, Horn P, Roth H, Schmiedek P, Vajkoczy P. Therapy of malignant intracranial hypertension by controlled lumbar cerebrospinal fluid drainage. Crit Care Med 2001; 29: 976-981.

2 Jennet B, Bond M. Assessment of outcome after severe brain damage. Lancet 1975; 2: $480-484$.

\section{C-10}

\section{Decompressive craniectomy in severe traumatic brain injury treated by an ICP targeted therapy}

M. Olivecrona ${ }^{1}$, M. Rodling-Wahlström² ${ }^{2}$ S. Naredi ${ }^{2}$, L.-O.D. Koskinen ${ }^{1}$

${ }^{1}$ Department of Neurosurgery, ${ }^{2}$ Department of Anestesiology and Intensive Care, University Hospital, Umeå, Sweden

Introduction: Severe traumatic brain injury (TBI) is one of the major causes of death in the younger age groups. In Umeå the "Lund concept" has been used in treatment for TBI since 1994. Since 1997 we have used decompressive craniectomy as a protocolled treatment step. The aim of the investigation is to study the effect of craniectomy on ICP over time during the ICU treatment and the outcome. 
Methods: Retrospective consecutive study. All patients treated for severe TB during 1st January 1998-31st December 2001 fulfilling the inclusion criteria i.e. GCS $\leqslant 8$ at intubation and sedation, a first recorded CPP $>10 \mathrm{mmHg}$, arrival within $24 \mathrm{~h}$ of trauma, and need of intensive care $>72 \mathrm{~h}$, were included. The patients were all treated according to the "Lund concept" and craniectomy performed when the ICP could not be controlled by evacuation of hematomas sedation, ventriculostomy, or low dose pentothal infusion. The cerebral intensive care was continued after the craniectomy. ICP, CPP, and MAP as all other vital parameters were continuously monitored. The study was approved by the local ethics committee.

Results: 93 patients met the inclusion criteria. Mean age 37.6 years 21 patients underwent craniectomy as a treatment step. The median GCS at intubation and sedation was 7 in the craniectomised group (DC) as well as in the non-craniectomised group. The mean treatment time before craniectomy after arrival to our hospital was $45 \mathrm{~h}$. The mean area of the craniectomy was $116 \pm 11 \mathrm{~cm}^{2}$ corresponding to a calculated volume of $124 \pm 14 \mathrm{~cm}^{3}$. We found a significant reduction of the ICP directly after craniectomy, from $36.4 \mathrm{mmHg}$ to $12.6 \mathrm{mmHg}$. The ICP was then followed for $72 \mathrm{~h}$. We observed an increase in ICP during the first 8-12 hours after craniectomy when it reaches approximately $20 \mathrm{mmHg}$, and levels out at $25 \mathrm{mmHg}$. The reduction of ICP is statistically significant for every point during the $72 \mathrm{~h}$. The outcome (GOS) in the craniectomised group was favourable (GOS 5-4) in 15 (71\%). Three (14\%) patients died within 3 months following trauma.

Conclusion: Craniectomy is a useful tool in achieving a lasting reduction in ICP in patients in whom the ICP not have been brought under control by the earlier steps in the treatment protocol. The effect over time of the procedure seems be satisfactory.

\section{Group D: Monitoring and Pathophysiology II}

\section{D-1}

\section{Attempts of improving accuracy of non-invasive ICP assessment}

B. Schmidt ${ }^{1}$, M. Czosnyka ${ }^{2}$, S.F. Bocklisch ${ }^{3}$, M. Päßler ${ }^{3}$, C. Leege ${ }^{4}$, J. Klingelhöfer ${ }^{1}$

${ }^{1}$ Department of Neurology, Medical Centre Chemnitz, ${ }^{2}$ Academic Neurosurgical Unit, Addenbrooke's Hospital, Cambridge,

${ }^{3}$ Department of Systems Theory, Technical University Chemnitz, and ${ }^{4}$ Department of Neurology, Technical University Munich

Introduction: In a formerly introduced mathematical model [1] signals of arterial blood pressure (ABP) and cerebral blood flow velocity (FV) were used to determine ICP non-invasively (nICP).

For that purpose some well defined hemodynamic parameters, computed from FV and ABP signals, were taken to calculate a linear signal transformation of ABP into ICP. In the current work attempts were made to optimise recent results by usage of advanced data analysis techniques.

Methods: In 201 patients (3-78 years, mean: $37 \pm 19$ years) with severe cerebral diseases (traumatic brain injury $N=176$, stroke $N=25$ ) signal data consisting of FV, ABP and (invasively assessed) ICP was used to compare ICP with nICP. Modifications of the existing linear nICP assessment model were made by means of a technique called Fuzzy Pattern Classification which allowed to create piecewise linear models. In a second approach Fuzzy Pattern Classification again was used for a pre-selection of the most suitable linear model. The results of these modified methods were compared to formerly achieved accuracy of nICP assessment using ICP as reference. Results: Two different linear models which were using different hemodynamic control parameters for nICP calculation were evaluated. On average of 201 patients the linear models showed mean absolute differences between $\mathrm{ICP}$ and $\mathrm{nICP}(\Delta \mathrm{ICP})$ of $6.41 \pm 5.74 \mathrm{mmHg}$ (median $=4.92 \mathrm{mmHg}$ ) and $6.39 \pm 5.76 \mathrm{mmHg}$ (median $=5.04 \mathrm{mmHg}$ ). The Fuzzy Pattern approach of piecewise linear model resulted in a $\Delta \mathrm{ICP}$ of $6.54 \pm 5.84 \mathrm{mmHg}$ (median $=5.29 \mathrm{mmHg}$ ). The three results did not differ significantly $(P>0.05)$. If Fuzzy Pattern Classification was used to control the application of the two linear procedures by continuously selecting the currently best procedure the $\Delta I C P$ could be reduced to $6.21 \pm 5.50 \mathrm{mmHg}$ (median $=4.83 \mathrm{mmHg})(P<0.001)$.

Conclusions: Although advanced methods of data analysis were used to improve the nICP procedure only minor reductions of nICP assessment errors could be achieved. It seems that the results are close to the limit of possible accuracy of ICP assessment by means of FV and ABP signal analysis.

Reference:

1 Schmidt $B$ et al. Adaptive non-invasive assessment of cerebral autoregulation and ICP. Stroke 2003; 34: 84-89.

\section{D-2}

Non invasive intracranial pressure evaluation by means of transcranial Doppler outside the ICU

E. De Peri ${ }^{1}$, M. Cortinovis ${ }^{1}$, A. Benigni ${ }^{1}$, A. Lavinio ${ }^{1}$, P. Liberini ${ }^{2}$, F. Rasulo ${ }^{1}$, A. Candiani ${ }^{1}$, N. Latronico ${ }^{1}$

${ }^{1}$ Institute of Anesthesiology - Intensive Care, ${ }^{2}$ Institute of Neurology, University of Brescia, Spedali Civili, Italy

Introduction: Brain injury is often associated with an increase of intracranial pressure (ICP) with consequent reduction of the cerebral perfusion pressure
(CPP). These parameters are easily obtained by means of invasive monitoring methods. Outside the intensive care unit (ICU) the non invasive estimation of CPP and ICP could be useful to guide the diagnostic and therapeutic conduct in patients at risk of developing intracranial hypertension. We used Transcranial Doppler (TCD) to non invasively estimate ICP in neurologic patients outside ICU.

Methods: ICP values measured through direct lumbar puncture (LP) (gold standard) were compared to ICP values obtained with TCD (index test) according to Czosnyka et al. [1].

Results: 27 patients were studied. Mean LP-ICP was 8.03 (SD 2.13) $\mathrm{mmHg}$. Mean TCD-ICP was 16.27 (SD 6.3) $\mathrm{mmHg}$. The average difference between the two measurements was 8.19 (SD 6.64) $\mathrm{mmHg}$ and was statistically significant ( $p$-value $<0.0001)$.

Conclusion: LP-ICP and TCD-ICP differ substantially. Future studies should evaluate if this is due to fact that TCD is inaccurate in measuring ICP or if TCD measures a different parameter.

Reference:

1 Czosnyka M, Basil F. Matta, Piotr Smielewski. Cerebral perfusion pressure in head injured patients: a non invasive assessment using transcranial Doppler ultrasonography. J Neurosurg 1998; 88: 802-808.

\section{D-3}

'ICM+': software for on-line analysis of data from bedside monitors in neurosurgical and neurointensive care patients

P. Smielewski ${ }^{1,2}$, M. Czosnyka ${ }^{1}$, S. Piechnik ${ }^{1}$, L. Steiner ${ }^{1}$, S. Momhjan ${ }^{1}$, J.D. Pickard ${ }^{1,2}$

${ }^{1}$ Academic Department of Neurosurgery, Addenbrooke's Hospital, Cambridge, UK, ${ }^{2}$ Wolfson Brain Imaging Centre, Addenbrooke's Hospital, Cambridge, UK

The ICM + software encapsulates almost 20 years of experience of brain monitoring gained in multiple international neuro-surgical and intensive care centres (Cambridge, Warsaw, Copenhagen, Geteborg, Detroit, Toulouse, and many others). The software collects data from bedside monitors using analogue and digital outputs and produces time trends of various summary parameters defined using mathematical functions of the input signals. It covers variety of aspects of multimodal bedside brain monitoring: ICP, arterial pressure, brain oxygenation, blood flow, neurotrending, brain temperature and many others. The on-line calculation engine allows trending of indices describing autoregulation of cerebral blood flow, cerebrospinal compensation and vascular reactivity. It allows calculation of non-invasive CPP. The post-processing tools support testing of transient hyperaemic response, leg-cuff autoregulation and $\mathrm{CO}_{2}$ reactivity. The software also incorporates various methods and tests used in diagnosis of hydrocephalus: overnight ICP monitoring, infusion study. An unique shunt database from the UK Shunt Evaluation Laboratory has been included to aid testing of performance of implanted shunts in-vivo.

The ICM + software enables modification of existing methods of brain monitoring and development of new algorithms through extensive programming of signal analysis. It aids the integration of physiological monitoring with clinical observations. In addition to time trends of calculated values and indices, the software also saves raw data from bed side monitors, which enables building up a library of signals for post-processing. These saved raw signals can be 
later processed by the on-line analysis engine thus providing means of testing newly invented indices and methods of on-line data processing.

Reference:

1 Czosnyka M, Whitehouse H, Smielewski P et al. Computer supported multimodal bed-side monitoring for neuro intensive care. Int J Clin Monit Comput 1994; 11: 223-232.

\section{D-4}

Cerebral haemodynamics assessed by transcranial Doppler ultrasonography during orthotopic liver transplant. A preliminary report

M. Czosnyka' ${ }^{1}$, E.A. Schmidt ${ }^{1,3}$, D. Tew ${ }^{2}$, J.R. Klink², M. Balestreri' ${ }^{1}$, J.D. Pickard ${ }^{1}$, B.F. Matta ${ }^{2}$

${ }^{1}$ Department of Neurosurgery, ${ }^{2}$ Anesthesiology, Addenbrooke's Hospital,

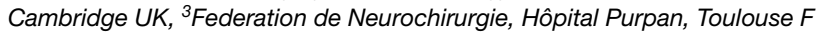

Background: Autoregulation is known to be altered in patients with acute liver failure $[1,2]$. Transcranial Doppler is considered as an appropriate technique to gauge cerebral haemodynamics during hepatic failure and liver transplantation $[3,4]$.

We aimed at assessing dynamic autoregulation [5] and non invasive cerebral perfusion pressure estimation (nCPP) [6] during an orthotopic liver transplantation (OLT).

Methods: OLT was performed in 10 patients suffering from chronic liver disease. General anesthesia was performed according to a standardized technique. During the surgery, as a routine clinical procedure, arterial blood pressure (ABP) was invasively measured and cerebral blood flow velocity was assessed using transcranial Doppler (TCD Intraview Rimed ${ }^{T M}$ ) in the middle cerebral artery. Analog signal was digitized, and stored in a computer using a dedicated in-house software. Off line, the correlation coefficient between $\mathrm{ABP}$ and FV, termed mean Mxa, was calculated [5]. Mxa close to +1 denotes that slow fluctuations in ABP produce synchronized slow changes in FV indicating defective autoregulation. In anesthetized patients, $\mathrm{Mxa}<0.4$ indicates a preserved autoregulation [7]. nCPP was also calculated as followed: $n C P P=\left(A B P m^{\star} F V d / F V m\right)+14$ [6]. During every surgery, we defined three time periods: the dissection phase ie from surgical start till portal vein has been clamped, the anhepatic phase when the portal vein is clamped and at least the reperfusion phase when the portal vein is released until the completion of the surgery. All haemodynamic indices were calculated for each time period and averaged.

Results:

\begin{tabular}{llll}
\hline & Dissection $(\mathbf{n}=\mathbf{1 0})$ & Anhepatic $(\mathbf{n}=\mathbf{1 0})$ & Reperfusion $(\mathbf{n}=\mathbf{9})$ \\
\hline ABP $(\mathrm{mmHg})$ & $78.4 \pm 9.7$ & $72.4 \pm 7.3$ & $66.9 \pm 9.3$ \\
nCPP $(\mathrm{mmHg})$ & $59.4 \pm 12.1$ & $60.1 \pm 10.4$ & $53.5 \pm 10.1$ \\
FV $(\mathrm{cm} / \mathrm{s})$ & $43.2 \pm 16.9$ & $46.1 \pm 14.4$ & $56.6 \pm 25.4^{+}$ \\
Mxa & $0.39 \pm 0.18$ & $0.49 \pm 0.17^{\star \star}$ & $0.63 \pm 0.16^{\star}$ \\
\hline
\end{tabular}

$\mathrm{ABP}$ was relatively stable during the surgery. $\mathrm{nCPP}$ followed passively the changes in ABP. Autoregulation significantly weakens in the course of the surgery, and deteriorates significantly whilst anhepatic $(p<0.01)$ the reperfusion phase $(p<0.001)$. Blood flow velocity increased significantly during reperfusion phase $(p<0.5)$.

Conclusion: We have been able to gauge non invasively cerebral haemodynamics during OLT. Our results indicate an alteration of dynamic autoregulation during the liver transplantation, and autoregulation deteriorates pari passu. During the reperfusion phase, autoregulation is severely deranged, indicating cerebral vasodilation. During liver transplant, nCPP looks maintained within a rather normal range, but further investigations are mandatory to assess the validity of this method during liver dysfunction.

\section{References:}

1 Larsen et al. Hepatology 1995; 22: 730-736.

2 Pott et al. Clin Physiol 1995; 15: 119-130.

3 Sidi et al. Anesth Analg 1995; 80: 194-200.

4 Doblar et al. J Clin Anesth 1993; 5: 479-485.

5 Czosnyka et al. Stroke 1996; 27(10): 1829-1834.

6 Czosnyka et al. J Neurosurg 1998; 71: 673-680.

7 Lang EW et al. J Neurol Neurosurg Psychiatry 2002; 72(5): 583-586.

\section{D-5}

\section{Monitoring and interpretation of intracranial pressure after} head injury

M. Czosnyka, M. Balestreri, L. Steiner, M. Hiler, P. Smielewski, P. Hutchinson, J.D. Pickard

Objective: To investigate the relationships between long-term computerassisted monitoring of intracranial pressure (ICP) and cerebral perfusion pressure (CPP) versus outcome, age, sex and time after injury.
Material and method: 429 sedated and ventilated head injured patients were continuously monitored from 1992 to 2002. ICP and arterial pressure (ABP) were recorded directly and stored in bed-side computers. Additional calculated variables included: 1 . Cerebral perfusion pressure $C P P=A B P-I C P$. 2. A pressure-reactivity index calculated as a moving correlation coefficient between slow waves (of period from $20 \mathrm{sec}$ to $3 \mathrm{~min}$ ) in ICP and ABP. 3 The magnitude of slow waves (of the periods longer than $20 \mathrm{sec}$ ).

Results: Fatal outcome was significantly associated with higher ICP $(p<0.000002)$, lower magnitude of slow waves $(p<0.00004)$, worse pressurereactivity $(p<0.0006)$ and lower CPP $(p<0.001)$. None of these parameters except for lower magnitude of slow waves $(p<0.03)$ were able to differentiate severely disabled patients from patients with favourable outcome. Worse outcome, lower mean ICP, worse pressure-reactivity and higher CPP were significantly $(p<0.05)$ were associated with the older age of patients. Higher averaged ICP, lower CPP, worse outcome and worse pressure reactivity were observed in females than in males (age-matched). Breakdown of the recorded parameters to years (from 1993 to 2001) indicated a step increase in CPP (from 70 to $80 \mathrm{mmHg}$ ) and decrease in ICP (from 20 to $17 \mathrm{mmHg}$ ) around 1997, due to the introduction of a uniform protocol for the expanded Neuro-Intensive Care Unit. It was followed by a decrease in mortality from $30 \%$ to $23 \%(p=0.041)$. The averaged profiles of ICP, CPP, PRx and slow waves presented as a function of time after injury showed no time-specific distribution in a whole group of patients studied.

Conclusion: High ICP and low pressure-reactivity are strongly associated with fatal outcome. There is a considerable heterogeneity amongst patientsoptimization of care depends upon observing the time-trends for the individual patient.

\section{D-6}

\section{Subdural monitoring of ICP during craniotomy in patients} without space-occupying lesions

L. Krogh, L. Jensen, T. Groefte, B. Bergholt, G.E. Cold

Department of Neuroanaesthesia, Aarhus University Hospital, Aarhus, DK-Denmark

Introduction: During craniotomy, opening of dura is especially critical. At high ICP opening of dura will inevitably result in brain swelling through the opening of dura. This has led to correlation studies of ICP measurements and the degree of cerebral swelling after opening of dura (1) in patients with space-occupying lesions. Cerebral swelling after opening of dura, does rarely occur at ICP $<5 \mathrm{mmHg}$. On the other hand, at ICP $>13 \mathrm{mmHg}$ cerebral swelling occurs with high probability. These ICP thresholds were independent of the pathophysiology, the anaesthetic agent and the $\mathrm{PaCO}_{2}$ level. No studies concerning the ICP in normal brains during anaesthesia are available. Material and methods: In 30 patients with either unruptured cerebral aneurysm $(n=19)$ or trigeminus neuralgi $(n=11)$, ICP was measured during craniotomy. All patients were anaesthetized with propofol/fentanyl. After removal of the bone flap and exposure of dura, ICP was measured subdurally with a $22 \mathrm{G}$ cannula connected via a water-filled polyethylene catheter to a pressure transducer. Simultaneously jugular bulb pressure (JBP) was measured in 11 patients. The degree of dural tension and cerebral swelling after opening of dura were also estimated.

Results: The median subdural pressure was $5 \mathrm{mmHg}$ (range $0-11 \mathrm{mmHg}$ ). Dural tension was estimated as normal in 27 patients, and increased in 3 patients. In only one patient swelling of the brain occurred after opening of the dura. ICP was not correlated to $\mathrm{PaCO}_{2}$, cerebral perfusion pressure or propofol maintenance dose, but significant correlations were found between weight of the patients and ICP $(\mathrm{ICP}=-0.12+0.063 \times$ weight, $\mathrm{P}=0.039$, $\mathrm{n}=30)$ and jugular bulb pressure and ICP $(\mathrm{ICP}=1.30+0.882 \times \mathrm{JBP}$, $\mathrm{P}=0.001, \mathrm{n}=11$ )

Conclusion: In patients without space-occupying cerebral lesions, anesthetized with propofol/fentanyl median ICP is $5 \mathrm{mmHg}$. Cerebral swelling of opening of dura occurs rarely. ICP correlates significantly to weight of the patients and to jugular bulb pressure.

\section{D-7}

Venous jugular bulb desaturation episodes during functional hemispherotomies

N. Fàbregas ${ }^{1}$, I. Bel ${ }^{1}$, L. Salvador ${ }^{1}$, R. Valero ${ }^{1}$, E. Carrero ${ }^{1}$, J. Rumià ${ }^{2}$

${ }^{1}$ Anesthesiology Department, Hospital Clínic, Barcelona University,

${ }^{2}$ Neurosurgical Service Hospital Clínic, Barcelona University

Introduction: There is a lack of available information about brain oxygenation during functional cerebral hemispherotomy procedures. We monitored 
venous jugular bulb saturation in patients with diffuse hemispheric disease scheduled for hemispherotomy.

Methods: We continuously recorded venous jugular bulb oxyhemoglobin saturation $\left(\mathrm{SjO}_{2}\right)$ [1], by means of a fiberoptic catheter inserted in the jugular vein ipsilateral to the healthy hemisphere. Other monitored parameters were: pulse oximetry, continuous arterial blood pressure, central venous pressure, urine output and end tidal $\mathrm{CO}_{2}$. BIS was measured in all patients except the first one. We analysed arterial blood gas and haemoglobin $(\mathrm{Hb})$ at different times depending on the intervention incidents. Clinical and demographical parameters were also recorded.

Results: We performed 5 functional hemyspherotomies for unilateral hemispheric disease and refractory epilepsy (one in 1998, two in 2000 and two in 2005) in three males and two females from 13 to 32 years old. Two patients had Rasmussen encephalitis, one had Parry Romberg disease and two, giant porencepahlic cysts. There were long procedures lasting from 7 hours to 9 hours $30 \mathrm{~min}$ (surgical time). No patient suffered severe haemorrhage. Initial $\mathrm{SjO}_{2}$ values in the first two patients were: $57 \%$ [mean arterial pressure (MAP) $87 \mathrm{mmHg}, \mathrm{PaCO}_{2} 38 \mathrm{mmHg}, \mathrm{PaO}_{2} 494 \mathrm{mmHg}, \mathrm{Hb} 13 \mathrm{~g} / \mathrm{dL}$ ] and $64 \%$ [MAP $81 \mathrm{mmHg}, \mathrm{PaCO}_{2} 28 \mathrm{mmHg}, \mathrm{PaO}_{2} 246 \mathrm{mmHg}, \mathrm{Hb} 13.9 \mathrm{~g} / \mathrm{dL}$ ]; maximal desaturation values respectively registered were: $44 \%$ [MAP $82 \mathrm{mmHg}$ $\mathrm{PaCO}_{2} 36.8 \mathrm{mmHg}, \mathrm{PaO}_{2} 223 \mathrm{mmHg}, \mathrm{Hb} 12 \mathrm{~g} / \mathrm{dL}$ ] and 21\% [MAP $77 \mathrm{mmHg}$, $\mathrm{PaCO}_{2} 24 \mathrm{mmHg}$ (with tidal volume of $8 \mathrm{~mL} / \mathrm{Kg}$ and respiratory rate (RR) of $\left.10 / \mathrm{min}), \mathrm{PaO}_{2} 238 \mathrm{mmHg}, \mathrm{Hb} 12 \mathrm{~g} / \mathrm{dL}\right]$. In the third patient the initial $\mathrm{SjO}_{2}$ value was $49 \%$ [MAP $70 \mathrm{mmHg}, \mathrm{PaCO}_{2} 34 \mathrm{mmHg}, \mathrm{PaO}_{2} 198 \mathrm{mmHg}, \mathrm{Hb}$ $13 \mathrm{~g} / \mathrm{dL}$ ]. In the fourth patient the initial $\mathrm{SjO}_{2}$ value was $38 \%$ [MAP $75 \mathrm{mmHg}$ $\mathrm{PaCO}_{2} 32 \mathrm{mmHg}, \mathrm{PaO}_{2} 272 \mathrm{mmHg}, \mathrm{Hb} 9.7 \mathrm{~g} / \mathrm{dL}$. After these findings we "intentionally" tried to hypoventilate mildly (tidal volume $8 \mathrm{~mL} / \mathrm{kg}$ and RR $10 / \mathrm{min}$ ) the last patient, the $\mathrm{SjO}_{2}$ value obtained was $80 \%$, but the $\mathrm{PaCO}_{2}$ was $51.4 \mathrm{mmHg}$, so we increased the RR to 12 and $\mathrm{SjO}_{2}$ went down to $63 \%$. All these desaturation episodes were related to minimal hyperventilation or slight decreases in blood pressure or with postural changes as antiTrendelenburg manoeuvre. This impairment in cerebral oxygenation would be missed without the specific monitoring.

All our patients have had a good neurologic outcome, one of them regaining speech capacity after disconnecting the left cerebral hemisphere. Conclusions: We unexpectedly detected several episodes of severe decrease of jugular saturation values in all our patients during these long neurosurgical procedures. In our opinion, the healthy cerebral hemisphere of these patients has an abnormal response to mild hypocapnia and hypotension [2], or has slight tolerance to perfusion pressure changes, being continuously at risk of cerebral hypoperfusion during the surgical procedure. Taking into account that functional cerebral hemispherotomies can last ten or twelve hours, there is a great probability of unnoticed intraoperative cerebral ischemia with a potential increase in postoperative morbidity and an impaired neurological outcome. It is important to confirm this finding, monitoring brain oxygenation during functional cerebral hemispherotomies.

References:

1 Matta BF, Lam AM, Mayber TS et al. Anesth Analg 1994; 79: 745-750.

2 Alfonso I, Vasconcellos E, Shuhaiber HH et al. J Child Neurol 2004; 19: 394-396.

\section{D-8}

Correlation between $\mathrm{AVDO}_{2}$ and arterio-venous difference in $\mathrm{CO}_{2}, \mathrm{pH}$, glucose, lactate and oxygen/glucose index in patients subjected to craniotomy

T. Groefte, K.I. Golbaekdal, L. Krogh, G.E. Cold

Department of Neuroanesthesia, Århus University Hospital, Denmark

Introduction: In recent studies a good positive correlation between $\mathrm{AVDO}_{2}$ respectively $\mathrm{SjO}_{2}$ and arterio-venous difference in $\mathrm{PCO}_{2}$ was documented, thus suggesting that diffusion of $\mathrm{CO}_{2}$ from tissue to venous blood is impeded, when $\mathrm{AVDO}_{2}$ is high. In the present study we tested the hypotheses that $\mathrm{AVDO}_{2}$ correlated to the arterio-venous difference of $\mathrm{pH}$, lactate and glucose, and the oxygen/glucose index.

Method: Paired samples of arterial and jugular bulb blood were analyzed for oxygen content, $\mathrm{PCO}_{2}, \mathrm{pH}$, glucose and lactate in patients subjected to craniotomy for cerebral tumor in propofol supplemented with fentanyl or remifentanil. The arterio-venous differences of oxygen $\left(\mathrm{AVDO}_{2}\right), \mathrm{PCO}_{2}\left(\Delta \mathrm{CO}_{2}\right)$, $\mathrm{pH}(\Delta \mathrm{pH})$, glucose ( $\Delta$ glucose), lactate ( $\Delta$ lactate) and oxygen/glucose index were calculated.

Results: The correlations between $\mathrm{AVDO}_{2}$ and respectively $\Delta \mathrm{CO}_{2}$ and $\Delta \mathrm{pH}$ were positive, highly significant with $\mathrm{P}<0.001$, and power 1.000 . The correlation between $\mathrm{AVDO}_{2}$ and $\Delta$ glucose, $\Delta$ lactate or the oxygen/ glucose index were insignificant (Figure indicates $P$ values, $r$ values and power).
Conclusion: Arterio-venous difference of $\mathrm{CO}_{2}$ and $\mathrm{pH}$ is highly correlated to $\mathrm{AVDO}_{2}$ while the arterio-venous difference of glucose lactate and oxygen/ glucose index are not.

\begin{tabular}{llll}
\hline & P values & Power & r values \\
\hline$\Delta \mathrm{CO}_{2}$ & $<0.001$ & 1.000 & 0.8249 \\
$\Delta \mathrm{pH}$ & $<0.001$ & 0.999 & 0.7123 \\
$\Delta$ glucose & 0.028 & 0.597 & 0.4147 \\
$\Delta$ lactate & 0.123 & 0.337 & 0.2983 \\
$\mathrm{O}_{2}$ /glucose index & 0.105 & 0.367 & 0.3131 \\
\hline
\end{tabular}

\section{D-9}

\section{Drift analysis of the Bowman regional cerebral blood flow monitor}

S. Wolf, L. Schürer, C.B. Lumenta

Department of Neurosurgery, Academic Hospital Munich-Bogenhausen, Technical University of Munich, Munich, Germany

Introduction: Bedside measurement of regional cerebral blood flow (rCBF) is a promising methodology to diagnose delayed ischemia after aneurysmal subarachnoid hemorrhage [1]. The Bowman thermodiffusion rCBF monitor offers quantitative assessment of brain tissue perfusion via algorithm-based differentiation of heat conduction and convective effects caused by brain blood flow.

Methods: The Bowman rCBF monitor was used in five patients after severe subarachnoid hemorrhage. The rCBF probe was implanted in the vascular territory of the aneurysm-harbouring vessel.

Automatic recalibration of the $\mathrm{CCBF}$ device was performed every $30 \mathrm{~min}$ utes. Continuous rCBF was stored once per second. Data was averaged over one minute and all measurement cycles pooled. Drift analysis was performed using analysis of variance for repeated measurements over the whole cycle and from beginning and ending sequence of each measurement cycle using the approach proposed by Bland and Altman.

Results: 1661 measurement cycles with a total monitoring time of 685 hours were analyzed. Mean rCBF was $33.7 \mathrm{ml} / 100 \mathrm{~g} / \mathrm{min}$ at the beginning and $31.2 \mathrm{ml} / 100 \mathrm{~g} / \mathrm{min}$ at the end of the measurement cycles between two recalibration periods, thus representing drift in the measurement of $2.5 \mathrm{ml} / 100 \mathrm{~g} / \mathrm{min}$ per 30 minute measurement period (ANOVA, $\mathrm{p}<0.001$ ). This drift was present regardless of initial CBF values and showed a logarithmic decline $(r=0.99, p<0.001)$.

Conclusion: Although the technology of regional CBF measurement is scientifically promising, the current implementation of the Bowman rCBF monitor shows a downward drift in the measurement cycles which is clinically relevant at low CBF states. As this drift follows a strong mathematical principle, modification of the algorithm for calculation of tissue perfusion from thermodiffusion data may improve the validity of the rCBF measurements.

\section{Reference:}

1 Vajkoczy P et al. Regional cerebral blood flow monitoring in the diagnosis of delayed ischemia following aneurysmal subarachnoid hemorrhage. JNS 2003; 98: 1227-1234.

\section{D-10}

\section{The radiographic validity of transcranial Doppler cerebral} blood flow velocity measurement

M.J. Souter ${ }^{1,2}$, G.W. Britz ${ }^{2,3}$, M.S. Kincaid ${ }^{1}$, B. Ghodke ${ }^{3}$, A.M. Lam ${ }^{1,2}$

Departments of ${ }^{1}$ Anesthesiology, ${ }^{2}$ Neurosurgery and ${ }^{3}$ Radiology, University of Washington, Seattle, WA, USA

Introduction: The validity of transcranial Doppler cerebral blood flow velocity (TCD) as an index of cerebral blood flow hinges on the assumption that the insonated cerebral vessel diameters do not change (outside the circumstances of vasospasm). This assumption has been challenged [1] particularly when considering the sensitivity and specificity of TCD to pathological and anesthesia induced changes in CBF. Accordingly we examined changes in both TCD flow velocities and angiographically derived cerebral artery diameters, in patients undergoing elective cerebral artery aneurysmal coiling, with crossover from a vasoconstrictor to a vasodilator anesthetic.

Methods: Ten patients were examined. Patients received a standard induction technique with propofol, paralytics and narcotics, and were stabilized on propofol infusion thereafter to control blood pressure within $20 \%$ of preanesthesia values. They were endotracheally intubated, with invasive arterial 
pressure monitoring, end-tidal and arterial $\mathrm{CO}_{2}\left(\mathrm{PaCO}_{2}\right)$ measurement Baseline measurements of middle cerebral artery (MCA) and internal carotid artery (ICA) flow velocities were made, along with radiographic measurements of cerebral arterial diameters at A1, M1, M2 and M3 segments. The anesthetic agent was changed to $2 \%$ isoflurane (end-tidal concentration) and measurements repeated. Blood pressure and arterial $\mathrm{CO}_{2}$ was held constant with changes in ventilation and the use of phenylephrine as required.

Results: The average change in mean arterial pressure (MAP) was $0.9 \%$ (SD 6.2) with a $\mathrm{PaCO}_{2}$ change of $0.5 \%$ (SD 3.7). The MCA and ICA experienced mean flow velocity increases of $6.1 \%$ (SD 8.9) and 25.2\% (SD 22.6), while the A1, M1, M2 and M3 diameters increased by 5.8\% (SD 7.0), 4.4\% (SD 7.5), 5.3\% (SD 7.9) and 6.7\% (SD 11.3) respectively. Changes were variable between patients, but every patient experienced a change in the diameter of at least one of the cerebral vessels insonated.

Conclusions: (i) Small changes in arterial diameter were seen accompanying similar changes in intracranial TCD flow velocities under conditions of controlled $\mathrm{pCO}_{2}$ and MAP. (ii) TCD blood flow velocity would appear to be a reasonable index of cerebral blood flow. However, previous assumptions that insonated conductance vessel diameters do not vary under anesthesia, may be questioned.

\section{Reference:}

1 Dahl A, Russell D et al. Simultaneous assessment of vasoreactivity using transcranial Doppler ultrasound and cerebral blood flow in healthy subjects. $J$ Cereb Blood Flow Metab 1994; 14: 974-981.

\section{Group E: Clinical Practice}

\section{E-1}

The significance of CT and detailed neurological examination for non-invasive evaluation of intracranial hypertension

S.V. Tsarenko, K.T. Guseynova, S.S. Petrikov, V.V. Krylov

Sklifosovsky Emergency Medicine Institute, Moscow, Russia

Introduction: The use of invasive probes is the best method of intracranial hypertension evaluation. Unfortunately it's associated with high costs, an increased risk of septic complications and non-availability of the equipment for intracranial pressure (ICP) measurement in non-specialized ICUs. Thus, the idea of this investigation was to find non-invasive methods of intracranial hypertension assessment that could substitute for the invasive ICP measurement.

Methods: 48 patients with Glasgo Coma Scale (GCS) $(\mathrm{M} \pm \mathrm{SD}) 8.1 \pm 2.1$ on admission were enrolled in the study (severe head injury $(n=32)$, arterial aneurysm rupture $(n=7)$, hemorrhagic stroke $(n=7)$, arterio-venous malformation rupture $(n=2))$. The average age was $44 \pm 16$. M/F ratio was 35/13. 26 patients underwent intracranial hematoma evacuation with decompressive craniotomy (DC), 13 - intracranial hematoma evacuation with boneplastic craniotomy (BPC), 9 - only insertion of ICP probe. ICP monitoring was provided during $4.8 \pm 3$ days with intraparenchemial microsensors (Codman) or ventricular systems (Hanni-Set). A CT scan and a neurological examination were done on admission and dynamically in pre- and postoperative periods. We considered on CT the size of zones with high and low density, midline shift as well as four stages of axial dislocation. We also distinguished four stages of brain dislocation according to brain stem segmental reflexes evaluation: the symptoms of diencephalon dysfunction - the 1st stage, mesencephalon - the 2nd, pons - the 3rd, medulla oblongata - the 4th ones.

Results: The comparision of ICP level obtained immediately after the beginning of the operation with preoperative GCS, CT scan and neurological symptoms of brain dislocation showed a good correlation between ICP and CT signs of axial dislocation $(r=0.59 ; p=0.0000 \ldots ; n=42)$ and midline shift $(r=0.43 ; p=0.004 ; n=42)$. We didn't find a good correlation between ICP values and GCS $(r=-0.02 ; p=0.196 ; n=48)$, neurological signs of brain dislocation $(r=0.27 ; p=0.07 ; n=42)$ and the size of zones with high and low density on CT scan $(r=0.3 ; p=0.06 ; n=30)$. In the postoperative period we compared ICP with clinical and CT data in patients with different types of neurosurgery procedure. After evacuation of hematoma and DC a good correlation was shown between ICP and GCS $(r=-0.47 ; p=0.007 ; n=30)$ and neurological signs of brain dislocation $(r=0.36 ; p=0.046 ; n=30)$. We found a poor correlation between ICP and CT scan data (the size of pathologic zones $(r=0.18 ; p=-0.44 ; n=21)$, midline shift $(r=-0.23 ; p=0.29 ; n=21)$ and CT signs of axial dislocation $(r=0.37 ; p=0.09 ; n=21))$. After evacuation of hematoma and BPC we found a good correlation between ICP and GCS $(r=-0.42 ; p=0.026$; $n=28)$ and CT signs of axial dislocation $(r=0.46 ; p=0.02 ; n=25)$. A poor correlation was found between ICP and neurological signs of brain dislocation ( $r=0.06 ; p=0.77 ; n=28)$, the size of zones with high and low density $(r=0.37 ; p=0.07 ; n=28)$ and midline shift $(r=0.35 ; p=0.09 ; n=25)$ on CT scan. Patients with multiple non-surgical hematomas and zones of contusion, who were operated only for ICP device insertion showed a good correlation between ICP and all evaluated parameters (GCS $(r=-0.79$; $p=0.0000 ; n=18)$, neurological signs of brain stem dislocation $(r=0.69$; $p=0.001 ; n=18)$, the size of pathologic zones on CT $(r=0.53 ; p=0.04$; $\mathrm{n}=15)$, midline shift $(r=0.69 ; p=0.003 ; n=15)$ and CT signs of axial dislocation $(r=0.57 ; p=0.02 ; n=15)$.

Conclusion: 1 . In the preoperative period intracranial hypertension could be estimated by evaluation of midline shift and axial dislocation on CT scan. The clinical picture wasn't informative presumably due to the nonhomogenity of the sample of the observed patients. 2. CT scan and neurological examination could be used for non-invasive intracranial hypertension evaluation in patients with multiple non-surgical hematomas and zones of contusion. 3. GCS and CT signs of axial dislocation were the best non-invasive criteria reflecting intracranial hypertension in patients with evacuated hematomas and bone-plastic craniotomy. 4. CT scan data weren't able to predict an ICP increase, but a neurological examination could be used effectively for this purpose in patients with evacuated hematomas and decompressive craniotomy.

\section{E-2}

Case report: the use of phenylephrine in the acute management of a patient with a suspected norepinephrine metabolism disorder

H. Akrawi, R. Lightfoot, C.A. Eynon

Wessex Neurological Centre, Southampton General Hospital, Southampton, United Kingdom

Introduction: The management of hypotension is a common occurrence on any intensive care. On our unit hypotension unresponsive to fluid challenges would normally be treated with norepinephrine. Patients with a genetic defect in the norepinephrine transporter (NET) have reduced reuptake of norepinephrine into the presynaptic terminal. We present a case of a patient with a suspected NET defect that was successfully treated with phenylephrine.

Case report: A 19-year-old female presented to her local hospital with a seven-day history of flu-like illness, nausea, vomiting and headache. Her Glasgow Coma Scale (GCS) was 3 on arrival. CT head showed brain oedema and meningeal enhancement consistent with meningo-encephalitis. Intravenous antibiotics and antiviral agents were started and an ICP monitor was inserted. Subsequent raised ICP lead to a repeat CT that revealed an acute right extradural haematoma $(E D H)$. She was transferred to the regional neurosciences centre for immediate evacuation of the EDH (the EDH was thought to be a complication of ICP monitor insertion).

Previously the patient had been investigated for suspected phaeochromocytoma and had undergone a bilateral adrenalectomy. In the year prior to this admission the patient had been investigated for orthostatic intolerance with a systolic blood pressure of $180 \mathrm{mmHg}$ on standing and a normal supine blood pressure. A provisional diagnosis of norepinephrine reuptake disorder had been made by measurement of plasma norepinephrine and its metabolites. Control of her blood pressure had been achieved with doxazosin and propanolol.

During this admission, hypotension and inadequate cerebral perfusion pressure (CPP) persisted despite fluid resuscitation. Doxazocin and propranolol had been discontinued for a week prior to admission due to the intercurrent illness. A continuous infusion of phenylephrine was commenced with rapid improvement in blood pressure and CPP. The patient went on to make a full recovery. The exact cause of her acute deterioration remains unclear. Discussion: Genetic deficits in the NET have only recently been described and are still incompletely understood. The influence of environmental factors 
results in poor correlation between specific deficits and phenotypes. Presentation can include tachycardia, weakness, fatigue, nausea, excessive sweating and altered mentation (generally occurring after standing). Phenylephrine acts specifically on the noradrenergic $a_{1}$ receptor and its metabolism is independent of the NET. In this case, the successful use of phenylephrine indicates that this patient's disease results from a reuptake problem at the presynaptic level.

References:

1 Mathias C, ed., Bannister R, ed. Autonomic Failure, a textbook of clinical disorders of the autonomic nervous system. 2001. Oxford University Press.

2 Tellioglu T, Robertson D. Genetic or acquired deficits in the norepinephrine transporter: current understanding of clinical implications. Exp. Rev. Mol. Med. 19 November 2001 http://www.expertreviews.org/01003878h.htm.

\section{E-3}

\section{BIS may reflect cerebral perfusion: case report during neuroendoscopic brain surgery}

P. Amorim ${ }^{1}$, M.P. Alonso ${ }^{1}$, M.J. Freitas ${ }^{1}$, J. Pamplona ${ }^{2}$, R. Pedroza ${ }^{2}$ A. Verdelho ${ }^{2}$, F. Lobo

${ }^{1}$ Serviço de Anestesiologia and Serviço de ${ }^{2}$ Neurocirurgia, Hospital Geral de Santo António, Porto, Portugal

Introduction: Cerebral procedures using neuroendoscopic techniques with irrigation through the endoscope have been associated with elevated intracranial pressure and neurological damage [1,2]. The use of an endoscope in stereotactic brain procedures is common. We describe a complication occurred during one such procedure and the usefulness of BIS monitoring to warn about possible damage.

Case report: A 54 years old patient, $90 \mathrm{~kg}, 170 \mathrm{~cm}$, was scheduled for stereotactic fenestration of a left temporal cyst of unknown aetiology (possibly inflammatory). He had hypertension and hiperuricemia. The patient presented hemiparesis and dysphasia. The GCS was 15 . He had already been subject to stereotactic drainage of a left temporal abscess, 2 months earlier. Pre-medication was with diazepam $10 \mathrm{mg}$ per os. Sedation was the anesthetic technique chosen. Oxygen, $40 \%$ was given with a Venturi mask throughout the entire case. Monitoring included EKG, non-invasive blood pressure, $\mathrm{SpO}_{2}, \mathrm{ETCO}_{2}$ (Datex AS3) and BIS (Aspect A2000XP). Blood pressure (BP) was $165 / 103 \mathrm{mmHg}$. Propofol was given by $\mathrm{TCl}$ using a Fresenius Orchestra pump with Schnider's PK model and effect site target. Sedation was achieved at a predicted effect site concentration of $1.4 \mu \mathrm{g} / \mathrm{ml}$. BIS was near 90 and the patient was arousable at verbal command. A Fischer ZD stereotactic frame was placed on the head following skin infiltration with $1 \%$ lidocaine. The CT showed a large temporal lobe cyst, a large area of oedema, compression of the left ventricle and a midline shift of $8 \mathrm{~mm}$. Following the CT scan, the patient was transferred to the OR; propofol effect site conc was changed in order to maintain adequate sedation (1.0 to $1.7 \mu \mathrm{g} / \mathrm{ml}$ ) . In the OR, the scalp was infiltrated with $1 \%$ lidocaine and a burr hole was performed. When spoken to or stimulated, the patient would open the eyes but remained comfortable and calm. The BIS remained around 85 and BP was stable around pre-anesthesia values.

When the endoscope was advanced into the brain a sudden decrease in BIS was observed. The propofol predicted effect site concentration had remained unchanged for around 20 minutes, the IV was running at constant $400 \mathrm{ml} / \mathrm{h}$ and no other drug had been administered. Heart rate and BP remained stable. The BIS stayed below 67 for 7 minutes; during this period the average BIS was 59.3 and minimum BIS was 52 . The drop in BIS was accompanied by a change in neurological status, and the patient became un-responsive. No changes in respiratory rate or $\mathrm{ETCO}_{2}$ were noticed. Blood gases confirmed that $\mathrm{PaCO}_{2}$ remained below 42. Propofol target concentration was lowered and the surgeon was informed. The endoscope was removed and 3 minutes later the BIS started to return to previous values, reaching 85 twelve minutes after the initial drop. At that moment the patient was obeying commands. A needle was then inserted and the temporal cyst was drained. The procedure was quickly completed. Total time of propofol administration was 179 minutes $(658 \mathrm{mg})$. Paracetamol, $1 \mathrm{~g}$, was given IV towards the end of the case. Upon skin dressing the BIS was around 90 and the patient's neurological status was similar to pre-operatively. A CT scan was immediately performed, showing reduction of the cyst volume, no midline shift and no blood clots. The immediate postoperative phase was uneventful. There was a marked improvement in the neurological deficits and no post operative complications occurred.

Conclusions: It is possible that insertion of the endoscope caused an increase in intracranial pressure, with a reduction of cerebral perfusion pressure and cerebral blood flow (CBF). The patient already had increased ICP and arterial hypertension, which made him more prone to a reduction in perfusion and flow at "normal" blood pressure values. The drop in BIS was definitely unrelated to anesthetic administration and most likely reflected a reduction in $\mathrm{CBF}$, coupled with a reduction in metabolism and hence in the BIS. The fact that sedation was used allowed us to confirm that a clinical neurological deterioration accompanied the drop in BIS. In this case, a BIS drop reflected a reduction in cerebral perfusion. BIS use may have contributed to an early detection of a possibly serious complication.

References:

1 J Neurosurg Aneth 2002; 88: 1170-1182.

2 J Neurosurg Aaneth 2004; 86: 24-33.

\section{E-4}

Neuroanesthetic practice in Catalonia, results of a National Survey

S. Fernández ${ }^{1}$, R. Valero ${ }^{1}$, A. Alcon ${ }^{1}$, N. Fábregas ${ }^{1}$, Grup ANESCAT ${ }^{2}$

${ }^{1}$ Department of Anesthesiology, Hospital Clínic de Barcelona, University of Barcelona, Catalunya Spain, ${ }^{2}$ Societat Catalana d'Anestesiologia, Reanimació i terapèutica del dolor, Acadèmia de Ciencies Mèdiques de Catalunya i Balears

Introduction: A survey of anaesthetic activity in Catalonia (North West Spain, 6 million inhabitants) was conducted in 2003, on behalf of the Catalan Society of Anaesthesiology (SCARTD), in order to quantify the workload and to describe common practices. We present the results focusing on neuroanesthetic activity.

Methods: A prospective and cross-sectional survey was performed for 14 randomised days during 2003. All public and private hospitals (131) practising anaesthesia around Catalonia participated in the survey. Data on characteristics of patients, anaesthetic techniques and type of procedure were included. We have analysed all procedures related to neuroanesthesia looking for demographic parameters, type of intervention, duration of procedures and postanaesthesia recovering characteristics.

Results: A total of 23.236 questionnaires were collected. Neurosurgical procedures (265), which represented $1.28 \%$ of the total anaesthesia activity, were performed in 28 Hospitals (12 public hospitals: $73.6 \%$ of procedures; 16 private hospitals: $26.4 \%$ procedures). This extrapolates 6909 neuroanesthetic procedures performed in Catalonia in 2003 (95\%IC 6022-7847).

Patients mean age was 48 years old (95\%IC 45.5-50.6); $52 \%$ were male, $48 \%$ female. ASA score was I: $22 \%$, II: $30.2 \%$, III: $31 \%$, IV-V: $16.9 \%$ Scheduled procedures were $79.7 \%$ and $20.3 \%$ were urgent procedures.

The most frequent interventions were: Spine surgery $40.1 \%$ of the procedures; Craniotomies for tumour resection $24.1 \%$, craniotomies for haemorrhage o trauma $7.2 \%$ (but $34.6 \%$ of the urgent procedures) and for aneurysms or AVM surgery $2.3 \%$; ventricular shunts $2.6 \%$.

Mean duration of the anaesthesia (including surgical time) was $150 \pm 108 \mathrm{~min}$. Mean duration of craneotomies for tumour resection was $287 \pm 95 \mathrm{~min}$.

All patients were distributed postoperatively in a conventional recovery room $(55.8 \%)$, in a monitored care unit $(19.2 \%)$ or in an intensive care unit (24.9\%). Craniotomy patients were admitted to a conventional recovery room $(24.7 \%)$, a monitored care unit $(33.7 \%)$ or an intensive care unit $(41.6 \%)$. Conclusions: About seven thousand Neurosurgical procedures were performed in Catalonia in 2003, in public (73.6\%) and private $(26.4 \%)$ hospitals. Spine surgery and craniotomies for tumour resection were the most frequently performed interventions. Craniotomy patients were mainly submitted to an ICU or a Monitored care unit.

\section{E-5}

\section{Survey of neuroanaesthesia practice patterns in Colombia}

\section{Arango ${ }^{1}$, C. Niño ${ }^{2}$, J. Mejia-Mantilla ${ }^{3}$}

${ }^{1}$ Department of Anesthesia, University of Western Ontario, Canada, ${ }^{2}$ Fundación Médica Santa Fe Bogotá, Colombia, ${ }^{3}$ Fundación Valle de Lili Cali, Colombia

Introduction: Neuroanaesthesia as a subspecialty is a relatively new in Colombia. Our objective was to assess current anaesthesia practice in Colombia with respect to patients undergoing cerebral supratentorial tumour (CST) resection and aneurysm clipping (AC).

Methods: In Nov 2004, a postal questionnaire survey was mailed to 48 hospitals (Level IV-V) throughout Colombia which provided care for adult neurosurgical patients on a routine basis. Questions on practice patterns included type of monitoring, anesthesia techniques, and use of hypertonic solutions, hyperventilation, systemic hypotension and hypothermia.

Results: $34 / 48$ hospitals responded (71\% response rate) by Jan 2005 . Of these, $13 / 34(38 \%)$ had established anaesthesia residency programs. However, only $2.9 \%$ of all cases were done by anaesthesiologists with a special interest in neuroanaesthesia. While only $3 / 34$ hospitals had a 
neurointensive care unit, 94\% used a surgical ICU for recovery instead of a PACU despite the fact that the majority of patients were extubated in the OR (78\% in CST and $61 \%$ in AC). For patients undergoing CST, we found that invasive arterial blood pressure (74\%) and central venous pressure (CVP) monitoring $(85 \%)$ were routinely used, however intracranial pressure (ICP) monitoring was used in only $6 \%$ of the hospitals. The most popular anaesthesia technique used was balanced volatile anaesthesia with opioids (88\%), less commonly was total intravenous anesthesia (TIVA) $(35 \%)$ and least common was volatile only in $6 \%$. No centre used $\mathrm{N}_{2} \mathrm{O}$. None of the centres used induced systemic mild hypothermia, but $88 \%$ administered steroids intraoperatively. $76 \%$ of hospitals used routine hyperventilation and $38 \%$ used induced systemic hypotension during CST. To reduce brain bulk, mannitol $20 \%$ was used in $97 \%$ of all hospitals and hypertonic saline in $32 \%$. Thiopental infusion was used in $71 \%$ to reduce ICP. Intraoperative magnesium measurement and replacement was routinely performed in 6\% of the hospitals. Normal Saline $0.9 \%$ was used in all patients in all hospitals for fluid replacement.

For patients undergoing AC, only $85 \%$ used invasive arterial blood pressure and $73 \%$ used CVP monitoring. Again the most popular anaesthesia technique used was balanced volatile anaesthesia with opioids (91\%), less commonly was TIVA (36\%) and $6 \%$ reported using $\mathrm{N}_{2} \mathrm{O}$. $44 \%$ of the hospitals reported using induced systemic mild hypotension routinely during $\mathrm{AC}$, $9.1 \%$ used intraoperative induced systemic hypothermia and $49 \%$ used thiopental infusions to reduce ICP.

Conclusion: We found that anaesthesia management for CST did not significantly differ from that for AC. Inhalational anaesthesia was the preferred technique. Despite recent literature refuting any improvement in neurological outcome with intraoperative mild hypothermia during AC [1], it continues to be used.. Induced hypotension and hyperventilation are prevalent practices during CST and AC. This survey shows that current neuroanaesthesia practices in Colombia may not follow recommended guidelines. The provision of neuroanaesthesia educational programs may help to improve existing standards.

\section{Reference:}

1 Todd MM, Hindman BJ, Clarke WR, Torner JC. Mild intraoperative hypothermia during surgery for intracranial aneurysm. N Engl J Med 2005; 352(2): 135-145.

\section{E-6}

Post ISAT pattern of management of 953 patients with intracranial vascular lesions in the West of Scotland

P. Harrison, I.E. Scott, V. Lochhead

Institute of Neurological Sciences, Southern General Hospital, Glasgow

Introduction: Since the publication of the International Subarachnoid Trial (ISAT) in the Lancet in 2001 confirmed a significant improvement in the mortality of coiled aneurysms compared to clippings at one year, the demand of the anaesthetic services for intracranial vascular lesions is changing dramatically from neurosurgical theatres to interventional radiology suites and recent publications have highlighted current provision of services in neurosurgical centres for interventional radiology $[1,2]$.

Aims: To quantify the change in demand for anaesthetic services for interventional radiological procedures and surgical interventions for intracranial vascular lesions from 2001 to 2005 within our regional tertiary referral centre for the Neurosciences in the West of Scotland.

Method: A retrospective data collection from logbook records from neurosurgical theatres and interventional radiology from 2001 to 2002 was undertaken. The data was anonymous. Ethical approval was not sought as it was a retrospective audit.

Results: Over the four years 2474 interventional radiological procedures, $739(30 \%)$ requiring general anaesthetic (GA) and 214 surgical craniotomies for intracranial vascular lesions were carried out. Of these $493(20 \%)$ were coil embolisations and $123(5 \%)$ were glue embolisations. Other interventions including balloon remodelling and particle embolisations requiring GA accounted for $123(5 \%)$. The total numbers radiological of procedures gradually reduced over 2001-2002 respectively being, 682, 626, 570, 596 however the percentage of interventions requiring $\mathrm{GA}$ increased from $14.8 \%$ to $28.6 \%$ to $37.2 \%$ to $41.4 \%$ from 2001 to 2005 respectively. In 2001 coil embolisations were 64/682(9\%), in 2002 121/626(19\%), 2003 150/570(26\%) and $2004158 / 596(27 \%)$ of the total although this may be misleading as actual numbers increased from 64 to 158 (more than double) within the four year period. Surgical craniotomies for clipping were 59 in 2001 and 65 in 2002 reducing to 31.29 in 2003 and 2004 respectively.

Conclusion: The current provision of services within the UK has recently been surveyed by Webb and Farling [2]. Within our regional tertiary referral centre the audit shows a dramatic shift in the demand for anaesthetic services out of the safety of the theatre environment and into the interventional radiology suites as is happening in the 34 Neurosurgical centres in the UK following the publication of the ISAT trial with its implications [1,2]. It is clear from the figures that there is a need to increase staffing equipment and training. Anaesthesia in each location is entirely different and the audit suggests a need for increasing the profile of training in anaesthesia for interventional radiology and all of its considerations including isolated site, point of care testing, access to the patient and radiation training.

\section{References:}

1 International Subarachnoid Aneurysm Trial (ISAT) of neurosurgical clipping versus endovascular coiling in 2143 patients with ruptured intracranial aneurysms: a randomised trial. The Lancet 2002; 360: 1267-1274.

2 Webb ST, Farling PA. Survey of arrangements for anaesthesia for neuroradiology for aneurismal subarachnoid haemorrhage. Anaesthesia 2005; 60(6).

\section{E-7}

\section{Patients with cervical discus prolaps being anaesthetised by nurse anaesthetists}

J. Foden, R. Stjernholm

Department of Neuroanaesthesia, Aarhus University Hospital

Introduction: In Denmark, nurses with special training, anaesthetise neurosurgical patients without direct supervision from consultant anaesthetists. The aim of this study was to investigate how many times consultant anaesthesiologists were called to the operating theatre and why, during operation for cervical discus prolaps.

Methods: Between April '04-December '04, a total of 71 patients undergoing this operation were recorded, of which 56 case histories were traced. Prior to the operation an anaesthesiologist visited all patients, and the decision whether the nurses were going to anaesthetise alone was taken. The following data was recorded: Anaesthetic drugs, difficult intubation, age, blood pressure (pre-, per- and post-operative), if there had been any use of consultant anaesthetists, and if so the reason.

Results: In 50 cases (89\%) anaesthesia was undertaken by nurse anaesthetists without further intervention by an anaesthesiologist. In the remaining 6 cases a consultant was called to the theatre to deal with one patient with low blood pressure and five cases of difficult intubation.

Conclusion: Patients with Cervical discus prolaps can be anaesthetised safely by nurses alone, provided that a consultant anaesthetist is always available.

\section{E-8}

\section{Correlation links between the length of peripherial nerves and the rate of their involvement into critical illness polyneuropathy (CIMP)}

A.A. Belkin, A.M. Alasheev

Clinical Institute of the Brain of the Ural branch of the Russian Academy of Medical Sciences, Yekaterinburg, Russia

Introduction: Critical illness polyneuromyopathy (CIPM) affects various peripheral nerves with different rate. The aim of our study is to investigate the correlation links between the length of peripheral nerves and the rate of their involvement into CIPM.

Methods: Prospective clinical trial of CIMP in neurosurgical and neurological patients was carried out on the basis of Clinical Institute of the Brain of the Urals department of the Russian Academy of Medical Sciences, Yekaterinburg, Russia. This research was approved by Ethical Committee of Clinical Institute of Brain. Patients older than 16 years with the clinical signs of systemic inflammatory response syndrome (SIRS), without diabetes mellitus and concomitant neuromuscular disease, during the first week of the mechanical ventilation were included into our research. The presence of CIPM was confirmed by clinical signs and electroneuromyography in all patients. The differentiation between polyneuromyopathy and myopathy was not provided. N. medianus, n. ulnaris, n. axillaries, n. tibialis, n. peroneus, n. femoralis, n. phrenic were investigated with the help of conventional standard stimulation electromyography. Nerve conduction velocity and amplitude compound muscle action potential were evaluated for the motor fibers.

Results: We investigated 43 patients (25 men and 18 women). 120 evaluation procedures were provided. Distribution of the rates of nerves involvement in CIMP is the following: n. medianus $71 \%(\mathrm{Cl} 61 \% ; 81 \%)$, n. ulnaris $85 \%$ (Cl 76\%; 92\%), n. axillaries 54\% (Cl 42\%; 65\%), n. tibialis 70\% (Cl 59\%; $79 \%$ ), n. peroneus $78 \%$ (Cl 66\%; 87\%), n. femoralis 54\% (Cl 42\%; 67\%), n. phrenic $51 \%(\mathrm{Cl} 40 \% ; 63 \%)$. The long nerves are more often involved in CIMP than the short ones $(p<0.001)$. Clinical signs and electromyographical information were equal. CIPM begins from the distal parts of low extremities and has ascending character. In the case of proximal parts of upper 
extremities involvement in CIMP, the respiratory alterations are strongly pronounced.

Conclusion: We found out some regularity of nerves damage rate in CIMP The longer is the nerve, the more often it is involved in CIMP.

\section{E-9}

Refractory status epilepticus in children: the role of propofol

J.P.J. van Gestel ${ }^{1}$, H.J. Blussé van Oud-Alblas ${ }^{1}$, M. Malingré ${ }^{2}$, F.F.T. Ververs ${ }^{2}$, K.P.J. Braun ${ }^{3}$, O. van Nieuwenhuizen ${ }^{3}$

${ }^{1}$ Pediatric Intensive Care Unit, ${ }^{2}$ Department of Pharmacy, ${ }^{3}$ Department of Pediatric Neurology, University Medical Centre Utrecht, Utrecht, The Netherlands

Introduction: Thiopental is commonly used as a last treatment in refractory status epilepticus (RSE). Because of adverse effects of thiopental, several guidelines recommend propofol as an alternative therapy. Propofol, however, is associated with potentially fatal outcome, and its efficacy in the treatment of RSE is almost exclusively based on small case series and case reports. The purpose of this study was to assess safety and efficacy of propofol and thiopental in the treatment of RSE in children.

Methods: Retrospective data collection of all children treated for RSE. Before 1999, thiopental was used, when phenytoin and high doses of midazolam had failed to terminate the status. After 1999, propofol was administered to all patients before thiopental was started, reserving thiopental for those who suffered from side-effects of propofol or did not respond to it The maximum dose of propofol never exceeded $5 \mathrm{mg} / \mathrm{kg} / \mathrm{h}$. Success and adverse effects of treatment were noted.

Results: In 14 out of 22 episodes treated with propofol (64\%), RSE could be adequately controlled. Side-effects of propofol were infrequent, of limited severity and fully reversible. Mortality was low and attributed to the severity of the underlying disorder. Seizure control with thiopental was achieved in
11 out of 20 children (55\%). During treatment with thiopental, a broad range of complications were encountered.

Conclusions: When correctly used, propofol is a safe and effective treatment for RSE in children. We suggest using propofol before thiopental.

\section{$E-10$}

Can electrocardiogram electrodes replace bispectral index electrodes for monitoring depth of anesthesia?

P. Akavipat, K. Dumrongbul, P. Neamnak

Department of Anesthesiology, Prasat Neurological Institute, Bangkok, Thailand

Introduction: To compare the feasibility of commercially available electrocardiogram (ECG) electrodes for the Bispectral index (BIS) monitoring.

Methods: We evaluated the difference in signal quality index (SQI) and BIS values collected from two BIS monitors, using both ECG electrodes and BIS electrodes in the same patients before anesthesia, during light anesthesia, deep anesthesia and emergence period. Both sets of electrodes were placed at bifrontal areas throughout the procedure. Statistical analysis was evaluated by bias with $95 \%$ limits of agreement and visualized by BlandAltman plot. Non parametric analysis was analyzed using paired t-test. A P-value less than 0.05 was considered statistically significant.

Results: There were 390 parallel signal quality index and BIS values recorded in this study. During anesthesia the mean BIS values were $58.63 \pm 18.77$ in ECG electrode group and $56.99 \pm 19.84$ in BIS electrode group, which were not statistically different. The mean SQI values were $79.20 \pm 24.77$ and 82.82 \pm 21.65 using ECG electrodes and BIS electrodes, respectively. The mean bias between BIS values was 1.65 with $95 \%$ limits of agreement between 0.91 and 2.38.

Conclusions: Commercial ECG electrodes could be used for monitoring depth of anesthesia with clinically acceptable mean bias and $95 \%$ limits of agreement of BIS value obtained from BIS electrode.

\section{Group F: Experimental Medicine and Pathophysiology}

\section{F-1}

Effects of recruitment maneuvers on cerebral and systemic hemodynamics in head trauma patients

E. Zavala, R. Adalia, F.J. Tercero, Ch. Heering, J. Roca

Surgical ICU, Anesthesiology Department, Hospital Clínic, Barcelona University, Barcelona, Spain

Introduction: Recruitment maneuvers are based on the application of high airway pressure to re-open alveolar units that previously were collapsed to improve oxygenation in acute lung injury (ALI/ARDS) patients and in patients with atelectasis formation during anesthesia. This RM may produce hemodynamic instability because the increase in airway pressure and decrease in cardiac output. It is also known that these patients can present respiratory failure due to chest injury, aspiration, nosocomial pneumonia or a neurogenic pulmonary edema. Consequently, therapeutic strategies have to combine treatment and prevention of cerebral ischemia as well as management of acute lung injury. The way this objective is reached still is controversial. The aim of this prospective study was to assess the effects of standardized recruitment maneuver (CPAP $40 \times 40$ ) on arterial oxygenation as well as in systemic and cerebral hemodynamics in head injury patients with ALI/ARDS Methods: We included 9 mechanically ventilated patients, aged $51.3 \pm 18$ $( \pm$ SD) years, GCS $9.2 \pm 3.1$ and with ALI/ARDS at an early stage $(24-72 \mathrm{~h}$ after ICU admission). Inclusion criteria were: age $>18$ years and diagnosis of ALI/ARDS. Exclusion criteria were: cardiogenic pulmonary and hemodynamic instability (mean arterial pressure (MAP) $<65 \mathrm{~mm} \mathrm{Hg}$ despite vasoactive drugs or volume replacement administration). One patient had ALI while the remaining 8 patients had ARDS, as defined by the American-European Consensus Conference. The protocol was approved by the ethics committee of the Hospital Clinic and informed consent was obtained from each patient's next of kin.

\begin{tabular}{lccc}
\hline Results & Basal & RM & 30 min. post RM \\
\hline $\mathrm{ICP} \mathrm{mm} \mathrm{Hg}$ & $11.0 \pm 7.0$ & $12.3 \pm 8.0$ & $10.7 \pm 7.0$ \\
$\mathrm{CPP} \mathrm{mm} \mathrm{Hg}$ & $86.5 \pm 5.0$ & $82.0 \pm 6.0$ & $79.3 \pm 6.0$ \\
$\mathrm{SjO}_{2} \%$ & $72 \pm 8$ & $69 \pm 3$ & $66 \pm 3$ \\
$\mathrm{PaO}_{2} \quad \mathrm{~mm} \mathrm{Hg}$ & $104.6 \pm 22.3$ & $163.8 \pm 50.7$ & $190.0 \pm 44.4$ \\
$\mathrm{PaCO}_{2} \mathrm{~mm} \mathrm{Hg}$ & $36.2 \pm 6.6$ & $36.7 \pm 11.8$ & $38.4 \pm 13.8$ \\
$\mathrm{MAP} \mathrm{mm} \mathrm{Hg}$ & $98.6 \pm 2.1$ & $90.0 \pm 1.0$ & $90.2 \pm 2.0$ \\
Pplat cm H & $24.2 \pm 3.0$ & $24.7 \pm 4.0$ & $22.9 \pm 4.0$ \\
Vt ml & $618 \pm 69$ & $616 \pm 109$ & $607 \pm 76$ \\
PEEP cm H $\mathrm{H}_{2} \mathrm{O}$ & $10.2 \pm 2.0$ & $10.9 \pm 2.0$ & $10.9 \pm 2.0$ \\
\hline
\end{tabular}

Conclusions: A RM (CPAP $40 \times 40)$ in head trauma patients conventionally ventilated, immediately improved arterial oxygenation without deleterious effects on cerebral hemodynamics and cerebral oxygenation and the effect lasted during 8 hours after RM was performed.

References:

1 Mascia $L$ et al. Acute lung injury in head trauma patient. Int Care Med 1998; 24: 1115-1116.

2 Bein T et al. Lung recruitment maneuver in patients with cerebral injury: effects on intracraneal pressure an cerebral metabolism. Int Care Med 2002; 28: 554-558.

\section{F-2}

\section{The effects of PEEP upon subdural ICP and CPP during} craniotomy

K.I. Golbaekdal, T. Groefte, B.U. Duch, N. Juul, G.E. Cold

Department of Anesthesia, Aarhus University Hospital, Denmark

Introduction: Adult respiratory distress syndrome (ARDS) develops in up to $20 \%$ of patients with severe head injury. Positive end-expiratory pressure (PEEP) is often required to support oxygenation. It is well known that PEEP might increase intracranial pressure (ICP) in intensive care patients, but the effects of PEEP, however, has not been investigated during craniotomy.

Methods: Subdural ICP, mean arterial blood pressure (MABP) and CPP were studied during application of $7 \mathrm{cmH}_{2} \mathrm{O}$ PEEP in 14 patients with cerebral tumors and 3 patients with subarachnoid hemorrhage. In 14 patients jugular bulb pressure (JBP) was measured simultaneously. The pressures were recorded one minute before and at $0,1,2$, and 3 minutes after PEEP application. Controlled ventilation was applied in all patients. The maximal respiratory pressure was recorded.

Results: The maximum respiratory pressure increased from 16 to $23 \mathrm{cmH}_{2} \mathrm{O}$. During the recording period MABP decreased from 71.5 to $68.6 \mathrm{mmHg}$ $(P=0.002)$ subdural ICP increased significantly from 5.3 to $6.7 \mathrm{mmHg}$ $(P<0.001)$, CPP decreased from 66.5 to $61.9 \mathrm{mmHg}(P<0.001)$, and JBP increased from 4.3 to $5.6 \mathrm{mmHg}(P=0.002)$. The increase in ICP was not correlated to the ICP level before PEEP application $(R=0.108, P=0.679)$. The linear regression for the relationship between increase in JBP $(\triangle \mathrm{JBP})$ and increase in ICP $(\triangle J B P)$ was found to be significant $(R=0.821, P<0.001)$. Conclusion: During craniotomy PEEP of $7 \mathrm{cmH}_{2} \mathrm{O}$ increases ICP and decreases MABP and CPP significantly. The increase in ICP is correlated to the increase in jugular bulb tension, but not to the level of ICP obtained just before PEEP application. 


\section{F-3}

Cerebral blood flow increases during infusion of mannitol in patients with acute bacterial meningitis

K. Møller ${ }^{1}$, F.S. Larsen ${ }^{2}$, P. Skinhøj ${ }^{1}$

Departments of ${ }^{1}$ Infectious Diseases and ${ }^{2}$ Hepatology, University Hospital Rigshospitalet, Denmark

Introduction: Osmotherapy using mannitol is frequently used in severely ill patients with bacterial meningitis and clinical signs of increased intracranial pressure, but its effect on cerebral haemodynamics is incompletely understood. We measured cerebral blood flow (CBF) during and after an infusion of mannitol in patients with bacterial meningitis.

Methods: The study was preceded by Institutional Review Board approval and informed consent from the subjects or their families. We included six mechanically ventilated adult patients with severe bacterial meningitis. Mannitol ( $1 \mathrm{~g}$ per $\mathrm{kg}$ body weight in five patients, $0.5 \mathrm{~g}$ per $\mathrm{kg}$ in one patient) was infused intravenously over 30 minutes. Cerebral blood flow velocity in the right middle cerebral artery $\left(V_{\text {mean }}\right)$ was measured using transcranial Doppler ultrasound during and up to 24 hours after infusion. Changes in CBF were further estimated by the Fick principle, by measuring the oxygen saturation in blood samples drawn from arterial and jugular bulb catheters, assuming that the global cerebral metabolic rate of oxygen was unchanged. Statistical analysis was performed using non-parametric testing and $P<0.05$.

Results: $V_{\text {mean }}$ increased rapidly and significantly during mannitol infusion and declined slowly thereafter, but remained above baseline levels for the entire study period in five of six patients. The arterial to jugular oxygen saturation difference $\left(\mathrm{a}-\mathrm{v} \mathrm{SO}_{2}\right)$ decreased during mannitol infusion and increased slowly after cessation of infusion, but remained below baseline levels in five of six patients. No rebound phenomena or other complications were observed.

Conclusion: A bolus infusion of mannitol increases cerebral blood flow in patients with acute bacterial meningitis. This effect is most pronounced during the first hour; however, the CBF elevation persists for several hours after infusion. A bolus infusion of mannitol appears to be safe in patients with severe bacterial meningitis, although its efficacy remains to be demonstrated in a controlled clinical trial.

\section{F-4}

Nitric oxide activity and cerebral blood flow effects of sodium nitroprusside and sodium nitrite

M.J. Souter ${ }^{1,3}$, J.D. Moulding ${ }^{1}$, S. Deem ${ }^{1,2}$, D. An², A.A. Artru ${ }^{1}$

Departments of ${ }^{1}$ Anesthesiology, ${ }^{2}$ Medicine and ${ }^{3}$ Neurosurgery, University of Washington, Seattle, Washington, USA

Introduction: Cerebral artery vasospasm is a significant source of morbidity following subarachnoid hemorrhage. Intraventricular (IT) administration of sodium nitroprusside has been anecdotally reported to relieve cerebral vasospasm, possibly through nitric oxide (NO) donation [1]. We compared the effect of two intraventricularly administered NO donors, sodium nitrite and sodium nitroprusside, upon cerebral blood flow (CBF) and NO activity, in a rabbit model. Sodium nitrite is less toxic than nitroprusside and may be a better NO donor in hypoxic circumstances [2].

Methods: The Institutional Animal Care and Use Committee approved the study. $11 \mathrm{New}$ Zealand white rabbits weighing 2.5-3.5 kg were anesthetized with $1-2 \%$ isoflurane allowing ear IV placement, and then received $15 \mathrm{mg} / \mathrm{kg}$ of ketamine and $0.33 \mathrm{mg} / \mathrm{kg}$ xylazine. Subsequently anesthesia was maintained with $1 \%$ isoflurane. Ventilation was adjusted to maintain a $\mathrm{pCO}_{2}$ of $35-45 \mathrm{mmHg}$. The animals were tracheostomized and both femoral arteries and a femoral vein cannulated. Temperature was controlled with rectal monitoring and a heat lamp. A catheter was placed into the left atrium via thoracotomy. The animals were mounted in a stereotaxic frame and an intraventricular needle placed through a burr hole $5 \mathrm{~mm}$ posterior and lateral to the bregma. The posterior neck muscles were dissected and a cisternal catheter placed through the atlanto-occipital membrane. A NO probe was calibrated and then placed through the cisternal cannula through which cerebrospinal fluid (CSF) was allowed to drain. Mock CSF was infused through the IT needle to replace CSF drained. A baseline CBF measurement was performed using colored fluorescent microspheres injected through the atrial catheter with simultaneous blood draws from the femoral catheters. Either sodium nitroprusside $(0.5 \mathrm{mg})$ or sodium nitrite $(0.5-2 \mathrm{mg})$ was then injected through the IT needle, and the CBF estimations were repeated at $20,90,150$ and 210-240 minutes. At 240 minutes the rabbits were killed and the brain and kidneys (reference organs) were extracted for subsequent blood flow analysis.

Results: Baseline CBF was $62 \pm 13.8 \mathrm{ml} / 100 \mathrm{~g} / \mathrm{min}$ (mean $\pm \mathrm{SD}$ ). Nitroprusside increased CBF by an average of $649 \%$ (range $357-1548 \%$ ) from baseline, with an effect persisting for 2 hours after administration. A dose of $2 \mathrm{mg}$ of sodium nitrite effectively increased CBF, with an average increase of $142 \%$ (range 33-334\%), for 90 minutes after injection. Arterial $\mathrm{pCO}_{2}$ varied within $1-2 \mathrm{~mm} \mathrm{Hg}$ from baseline. End-tidal isoflurane was held constant at 0.8 to $0.9 \%$. Nitric oxide activity increased by $160 \pm 103 \mathrm{nM}$ after injection of nitroprusside and by $90 \pm 31 \mathrm{nM}$ after nitrite. The NO increase started from 16-25 minutes post injection and lasted from 1-2 hours with a greater duration of effect from nitroprusside. Neither agent induced systemic hypotension. Summary: Nitroprusside and nitrite both induce durable increases in CBF after IT administration, concurrent with the generation of NO activity in CSF. This may prove of benefit in patients with cerebral vasospasm.

References:

1 Thomas JE et al. Stroke 1999; 30(7): 1409-1416.

2 Gladwin, MT. J Clin Invest 2004; 113(1): 19-21.

\section{F-5}

The impact of open lung ventilation on intracranial pressure in neurosurgical patients

S. Wolf ${ }^{1}$, D.V. Plev ${ }^{2}$, L. Schürer ${ }^{1}$, H.A. Trost ${ }^{1}$, C.B. Lumenta ${ }^{1}$

${ }^{1}$ Department of Neurosurgery, Academic Hospital Munich-Bogenhausen, Technical University of Munich, Munich, Germany and

${ }^{2}$ London Spine Clinic, London, UK

Introduction: The appropriate standard of care for patients with acute respiratory distress syndrome (ARDS) is ventilation with low tidal volumes around $6 \mathrm{ml} / \mathrm{kg}$ body weight to limit ventilation-induced lung injury, combined with sufficient positive endexpiratory pressure (PEEP) to prevent alveolar collapse [1]. Additionally, short periods of sustained high inflation pressure termed recruitment maneuvers, may reopen collapsed lung tissue. We report on our experience with this open lung approach in neurosurgical patients with acute brain lesions which were not included so far in mayor ARDS studies due to concerns on intracranial pressure (ICP) rise.

Methods: 31 patients with acute intracranial pathology, predominantly subarachnoid hemorrhage and traumatic brain injury, and concomitant ARDS were examined on the ventilatory parameters and on their course of ICP before and after initiation of open lung ventilation. Recruitment maneuvers were performed with an increase of PEEP to at least $15 \mathrm{mmHg}$ and ventilation with an inspiratory pressure up to $40 \mathrm{mmHg}$ over PEEP for a few breathing cycles.

Results: 24 hours following initiation of open-lung ventilation, the mean oxygenation index improved from 132 to 280, thus indicating nearnormal gas exchange. The mean PEEP level used was $11 \mathrm{mmHg}$ before, $17 \mathrm{mmHg}$ immediately after and $14 \mathrm{mmHg} 24$ hours after initiation of openlung ventilation. Three patients failed improvement of oxygenation after recruitment.

Mean ICP was $14.0 \mathrm{mmHg}$ before and $13.7 \mathrm{mmHg}$ after 24 hours with a maximum of $18.3 \mathrm{mmHg}$ during and shortly after recruitment. Nine patients needed additional ICP treatment related to open-lung ventilation, but in none it had to be aborted due to intractable ICP rise. Patients with prior low ICP showed a discrete increase in ICP after initiation of open-lung ventilation, while most patients with preceding critically elevated ICP showed a decrease in mean ICP level.

Conclusion: According to our experience, there is no reason to exclude neurosurgical patients with acute brain lesions from open lung ventilation. The potential risk of intracranial deterioration is low and is justified by the advantage of improved and adequate oxygenation.

Reference:

1 Marini J, Gattinoni L. Ventilatory management of acute respiratory distress syndrome. A consensus of two. Crit Care Med 2004; 32: 250-255.

\section{F-6}

Intraarterial infusion of nimodipine for the treatment of cerebral vasospasm following subarachnoid hemorrhage (SAH)

K. Bülow ${ }^{1}$, J. Nepper-Rasmussen², F. Gaarskjaer ${ }^{3}$

${ }^{1}$ Department of Anaesthesia and Intensive Care, ${ }^{2}$ Department of Neuroradiology, ${ }^{3}$ Department of Neurosurgery, Odense University Hospital, Odense, Denmark

Introduction: Cerebral vasospasms cause significant morbidity and mortality following SAH and are still a challenging problem. Nimodipine (NIM), a dihydropyridine calciumantagonist, is administered orally or intravenously to most patients following SAH to prevent vasospasms and thus improve outcome. Recently, intraarterial infusion of NIM has been proposed [1]. In our department we routinely use balloon-angioplasty for the treatment of cerebral vasospasms. We here present the retrospective review of 14 anaesthesia charts representing 9 patients treated with intraarterial NIM alone or in 
combination with balloon-angioplasty. Special attention is paid to the hemodynamic response to NIM-infusion.

Methods: Anaestetic charts of 9 consecutively treated patients and a total of 14 infusion-procedures were reviewed. Patients were included following a multifactorial algorithm based on progressive deterioration and/or progressive focal neurological deficit, despite maximal medical treatment. Al patients had raised flow velocity measured with transcranial ultrasound Doppler.

All patients were treated in general anaesthesia with Sevoflurane, fentanyl and rocuronium. 2 to $4 \mathrm{mg}$ of NIM were infused over 30 minutes into the affected arteries. The treatment was repeated, in case the patients clinical status did not improve within 24 hours and a new angiography demonstrated vasospasms. In these cases infusion of NIM was combined with balloon angioplasty. Postoperatively the patients received intravenous infusion of NIM $30 \mathrm{microgr} / \mathrm{kg} / \mathrm{h}$ for 7 days.

Results: Clear angiographic effect was observed in all cases. Infusion of Noradrenalin $0.1-0.2 \mathrm{microgr} / \mathrm{kg} / \mathrm{min}$ with supplemental doses of metaoxedrin was given in all cases to keep the blood pressure at pre-treatment level. Hemodynamic stability was easily achieved by titration of the vasopressor infusion rate. In one patient, receiving labetalol for hypertension, the mean arterial blood pressure decreased more than $30 \mathrm{~mm} \mathrm{Hg}$. Otherwise, no complications were observed perioperatively. Eventually, one patient died due to causes unrelated to the treatment. Two patients developed focal neurologic deficit due to vasospasms or SAH. Six patients survived with no focal neurological deficit.

Conclusions: Intraarterial infusion of $2-4 \mathrm{mg}$ NIM over 30 minutes with adjunctive infusion of alpha-agonists is safe and associated with hemodynamic stability. The treatment resolves vasospasms as demonstrated by angiography. The time-window for treatment, dose and route of administration of NIM as well as the combination with balloon angioplasty is still an issue of debate.

Reference:

1 Biondi A et al. Intraarterial nimodipine for the treatment of symptomatic cerebral vasospasm after aneurysmal subarachnoid hemorrhage: preliminary results. $A m \mathrm{~J}$ Neuroradiol 2004; 25: 1067-1076.

\section{F-7}

\section{Prostacyclin treatment of nimodipine resistant cerebral} vasospasm - a pilot study

L.-O.D. Koskinen ${ }^{1}$, M. Olivecrona ${ }^{1}$, M. Rodling-Wahlström ${ }^{2}$, S. Naredi ${ }^{2}$

Departments of ${ }^{1}$ Neurosurgery and ${ }^{2}$ Anaesthesiology and Intensive Care, Umeå University Hospital, Umeå, Sweden

Introduction: Cerebral vasospasm is a major problem in some patients suffering subarachnoid haemorrhage (SAH). Several treatment approaches aiming at reducing the vasospasm are available. Some of these are the use of peroral or intravenous nimodipine (Nimotop ${ }^{\circledR}$ ) medication, $\mathrm{HHH}$-therapy, endovascular angioplasty, intra-arterial nimodipine and nitroprusside. An interesting ongoing clinical trial with an endothelin receptor inhibitor waits for its results. Prostacyclin, an endogenous substance with vasodilatatory properties and inhibiting effects on platelet aggregation and leukocyte adhesion to the endothelium, is from a theoretically point of view interesting. Actually, already about 20 years ago prostacyclin was shown to be effective in reducing cerebral vasospasm. Prostacyclin has also been used in the treatment of kidney diseases, pulmonary hypertension and experimentally in severe traumatic brain injury.

Methods: The present study is a prospective open pilot study. Inclusion criteria were proven SAH due to ruptured aneurysm, development of vasospasm as defined by transcranial Doppler (TCD) measurement with mean flow velocity (MFV) exceeding $120 \mathrm{~cm} / \mathrm{s}$ in MCA. Further, the vasospasm should be resistant to high dose i.v. $(15 \mathrm{ml} / \mathrm{h}=3 \mathrm{mg} / \mathrm{h})$ nimodipine infusion. All patients were sedated due to unconsciousness or high ICP, artificially ventilated and treated at the ICU. ICP, CPP and all vital parameters were continuously monitored. The preset goal of CPP level was $70 \mathrm{mmHg}$ and ICP $<20 \mathrm{mmHg}$. Patients were initially treated with a prophylactic nimodipine infusion $(2 \mathrm{mg} / \mathrm{h})$. When vasospasm was observed the nimodipine dosage was increased to $3 \mathrm{mg} / \mathrm{kg}$. Epoprostenol (Flolan, GlaxoSmithKline) was infused i.v. in a dose of $0.5 \mathrm{ng} / \mathrm{kg} / \mathrm{min}$ during 72 hours if the vasospasm was not successfully treated by nimdoipine. Values are reported as mean \pm s.e.m. or median. The local ethics committee approved the study.

Results: Five patients fulfilled the criteria, 3 female and 2 male. Mean age was $49 \pm 6 y$ r, Hunt-Hess 4 , Fisher 4 . Typically, the vasospasm developed a few days after the SAH. The MFV was $163 \pm 24 \mathrm{~cm} / \mathrm{s}$ during the period of $2 \mathrm{mg} / \mathrm{h}$ nimodipine infusion. Nimodipine infusion was increased to $3 \mathrm{mg} / \mathrm{h}$. The MFV was $199 \pm 31 \mathrm{~cm} / \mathrm{s} 24$ hours later. In this situation the epoprostenol infusion was started and 48 hours later the MFV was $122 \pm 27 \mathrm{~cm} / \mathrm{s}$ and after 72 hours $92 \pm 6 \mathrm{~cm} / \mathrm{s}$. Although the material is very small the effect is statistically significant as evaluated by the Kruskal Wallis test $(p=0.048)$. At 3 -month follow-up the median GOS was 4 and at 12-months 4

Conclusions: Cerebral vasospasm is still a major cause of morbidity and mortality in SAH. Partially effective ICU regimes and pharmacological treatments have improved the outcome but no absolute preventive measures for vasospasm are available. High dose nimodipine usually decreases the MFV within 24 hours. This was not the case in these patients. In the present study we are able to show that low dose epoprostenol may have a beneficial effect in reducing established nimodipine resistant vasospasm. Indeed, a prospective randomized blinded study is needed to definitely show whether the effect of epoprostenol can reduce vasospasm after SAH.

\section{F-8}

\section{Effect of norepinephrine on brain $\mathrm{SO}_{2}$ compared with dobutamine}

K. Sato ${ }^{1}$, H. Kamii ${ }^{1}$, H. Shimizu ${ }^{2}$, M. Kato ${ }^{3}$

Departments of ${ }^{1}$ Neuroanesthesia, ${ }^{2}$ Neurosurgery, Kohnan Hospital, ${ }^{3}$ Department of Anesthesiology and Perioperative Medicine, Tohoku Postgraduate Medical School, Sendai, Japan

Introduction: Maintenance of systemic blood pressure and cerebral perfusion pressure is essential in cross clamping in carotid endarterectomy (CEA) and vasopressors are often required. Reported cerebral effects of norepinephrine are variable $[1,2]$ and intraoperative use of norepinephrine has been the subject of much controversy. This study investigated the effect of norepinephrine and dobutamine on brain regional $\mathrm{SO}_{2}\left(\mathrm{rSO}_{2}\right)$ measured by infrared spectroscopy.

Methods: Fifteen patients undergoing CEA were included with institutional ethics committee approval and informed consents. The ages ranged from 55 to 76 , mean 67.1 years and 2 females and 13 males were included. Bilateral frontal $\mathrm{rSO}_{2}$ was monitored with transcranial optical spectroscopy, TOS-96 (Tostec Co., Tokyo). Anesthesia was induced and maintained with propofol, fentanyl and vecuronium. After 10 minutes of steady state in terms of anesthesia and systemic circulation, norepinephrine was infused toward the target $\mathrm{BP}$, averaged MAP measured in ward, up to $0.1 \mathrm{mcg} / \mathrm{kg} / \mathrm{min}$ or dobutamine was administrated toward the target $\mathrm{HR}$, averaged $\mathrm{HR}$ measured in ward, up to $5.0 \mathrm{mcg} / \mathrm{kg} / \mathrm{min}$ via central venous line. After $10 \mathrm{~min}$ steady state measurements, surgery was initiated.

Results: Norepinephrine study was performed in 7, dobutamine study in 8 and the target MAP or HR was achieved in every case. There were no patients with ECG change during norepinephrine or dobutamine infusion. The dose of norepinephrine was $0.02-0.08$, mean $0.04 \mathrm{mcg} / \mathrm{kg} / \mathrm{min}$ to achieve the target BP. MAP increased from $71.4 \pm 11$ to $96.5 \pm 8.9 \mathrm{mmHg}$ $($ mean $\pm S D)(P<0.001), H R$ decreased from $56.0 \pm 6.9$ to $48.4 \pm 4.5 \mathrm{bpm}$ $(\mathrm{P}<0.001)$. Despite the MAP increase, ipsilateral and contralateral $\mathrm{rSO}_{2}$ decreased from $70.7 \pm 4.8$ to $68.6 \pm 4.2(P<0.01), 71.3 \pm 4.5$ to $69.4 \pm$ $3.9 \%(\mathrm{P}<0.05)$ respectively. The dose of dobutamine was 1.0-3.0, mean $1.6 \mathrm{mcg} / \mathrm{kg} / \mathrm{min}$ to achieve the target HR. MAP and HR increased from 76.3 \pm 9.8 to $81.8 \pm 5.4 \mathrm{mmHg}$ (NS), from $61.6 \pm 7.9$ to $67.0 \pm 12.4 \mathrm{bpm}$ (NS). Ipsilateral and contralateral $\mathrm{rSO}_{2}$ increased from $67.0 \pm 5.5$ to $68.4 \pm 4.8$ (NS), $65.9 \pm 5.7$ to $66.8 \pm 5.7 \%$ (NS) respectively.

Conclusion: Norepinephrine to maintain the target MAP decreased brain $\mathrm{rSO}_{2}$. Norepinephrine, but not dobutamine would aggravate the cerebral circulation in patients undergoing CEA. Further investigations are necessary to clarify the effect of norepinephrine on cerebral circulation.

References:

1 Reynier-Rebuffel AM et al. Nonuniformity of CBF response to NE- or ANG II-induced hypertension in rabbits. Am J Physiol 1987; 253: H47-H57.

2 Olesen J. The effect of intracarotid epinephrine, norepinephrine, and angiotensin on the regional cerebral blood flow in man. Neurology 1972; 22: 978-987.

\section{F-9}

\section{Cerebral metabolic improvement following hypertonic saline administration in patients with severe subarachnoid haemorrhage}

P.G. Al-Rawi ${ }^{1}$, M.-Y. Tseng ${ }^{1}$, J. Nortje ${ }^{2}$, B.F. Matta ${ }^{2}$, P.J. Hutchinson ${ }^{1}$, P.J. Kirkpatrick

University Departments of ${ }^{1}$ Neurosurgery and ${ }^{2}$ Anaesthesia, Addenbrooke's Hospital, Cambridge, UK

Introduction: Following aneurysmal subarachnoid haemorrhage (SAH), cerebral blood flow (CBF) may be reduced, resulting in poor outcome due to cerebral ischaemia and subsequent stroke. Hypertonic saline (HS) is known 
to be effective in reducing ICP. We have previously shown a $20-50 \%$ increase in $\mathrm{CBF}$ in ischaemic regions after intravenous infusion of hypertonic saline [1]. The aim of this study is to explore whether cerebral blood flow (CBF) enhancement with hypertonic saline (HS) in patients with severe subarachnoid haemorrhage $(\mathrm{SAH})$ is associated with an improved oxygenation and metabolic profile.

Methods: After Local Research Ethics Committee approval and following informed assent, 28 patients (age range 37-74) suffering from poor grade $\mathrm{SAH}$ underwent continuous bedside multimodal monitoring of ABP, ICP, CPP, brain tissue oxygen $\left(\mathrm{PtiO}_{2}\right)$, middle cerebral artery flow velocity (FV), and microdialysis. Quantified xenon computerised tomography (XeCT) was performed before and after infusion of $23.5 \% \mathrm{HS}$, and CBF in a region of interest around the brain oxygen and microdialysis probes was calculated (ROI CBF). Data is given as mean $\pm \mathrm{SE}$.

Results: Average baseline ROI CBF was $31.6 \pm 3.6 \mathrm{ml} / 100 \mathrm{~g} / \mathrm{min}$. In 4 patients, $\mathrm{ROI}$ CBF decreased after $\mathrm{HS}$. In the remainder, a significant increase in ROI CBF was observed ( $p<0.05$ ). Pooling the data for all patients, at 30 minutes post $\mathrm{HS}$ infusion a significant increase in ABP, CPP, FV and $\mathrm{PtiO}_{2}$ was seen, together with a significant decrease in ICP $(p<0.05)$. At 60 minutes ICP, CPP, FV and $\mathrm{PtiO}_{2}$ still showed significant changes $(p<0.05)$, blood pressure returned to baseline levels and a significant increase in glucose was seen. In 18 patients, a decrease in lactate-pyruvate ratio was seen at 60 minutes following HS. No side effects have been observed.

Conclusion: HS safely and effectively augments CBF in patients with poor grade SAH and significantly improves cerebral oxygen and glucose. An improvement in cerebral metabolic status in terms of lactate-pyruvate ratio is also associated with HS infusion.

Reference:

1 Tseng MY, Al-Rawi PG, Pickard JD et al. Effect of hypertonic saline on cerebral blood flow in poor grade patients with subarachnoid haemorrhage. Stroke 2003; 34: 1389-1397.

\section{F-10}

Effect of ischemic preconditioning on brain tissue gases and pH during temporary cerebral artery occlusion

M.T.V. Chan ${ }^{1}$, R. Boet ${ }^{2}$, S.C.P. $\mathrm{Ng}^{2}$, W.S. Poon ${ }^{2}$, T. Gin ${ }^{1}$

${ }^{1}$ Department of Anaesthesia and Intensive Care and ${ }^{2}$ Division of Neurosurgery, Department of Surgery, The Chinese University of Hong Kong, Hong Kong

Background: We evaluated the effects of ischemic preconditioning, produced by 2 min proximal temporary artery occlusion, on brain tissue gases and acidity during clipping of cerebral aneurysm.

Materials and Methods: This study was approved by ethics committee. Twelve consenting patients with aneurysmal subarachnoid hemorrhage were recruited. All patients received standard anesthetics. After craniotomy, a calibrated multiparameter sensor (Neurotrend, Diametrics Medical, Minneapolis, $\mathrm{MN}$ ) was inserted to measure oxygen $\left(\mathrm{PtO}_{2}\right)$ tension, carbon dioxide $\left(\mathrm{PtCO}_{2}\right)$ tension and $\mathrm{pH}(\mathrm{pHt})$ in tissue at risk of ischemia during temporary artery occlusion. In patients assigned to the preconditioning, proximal artery was occluded initially for $2 \mathrm{~min}$ and was allowed to reperfuse for $30 \mathrm{~min}$. All patients underwent cerebral artery occlusion for clipping of aneurysm. The rate of change in $\mathrm{PtO}_{2}, \mathrm{PtCO}_{2}$ and $\mathrm{pHt}$ after artery occlusion were compared between groups using unpaired $t$ test. $P<0.05$ was considered significant.

Results: Baseline brain tissue gases and $\mathrm{pHt}$ (mean $\pm \mathrm{SD}$ ) were similar between groups. Following artery occlusion, the decline in $\mathrm{PtO}_{2}$ and $\mathrm{pHt}$ were significantly slower in the preconditioned group.

Conclusions: These results suggested that ischemic preconditioning attenuates tissue hypoxia during subsequent artery occlusion.

\section{Group G: Disease Mechanisms}

\section{G-1}

The role of apolipoprotein E polymorphism in the course of subarachnoidal haemorrhage

L.Z. Csajbok ${ }^{1}$, M. Öst ${ }^{1}$, K. Nyhlén ${ }^{2}$, K. Blennow ${ }^{3}$, B. Rydenhag ${ }^{4}$, B. Nellgård ${ }^{1}$

${ }^{1}$ Department of Anesthesiology and Intensive Care Medicine,

${ }^{2}$ Department of Neurology, ${ }^{3}$ Department of Neurochemistry,

${ }^{4}$ Department of Neurosurgery, Sahlgrenska University Hospital, Gothenburg, Sweden

Background and Purpose: Aneurismal subarachnoidal haemorrhage (SAH) is still associated with high morbidity and mortality although novel treatments are now offered. Genetic variability may explain outcome differences after SAH. Apoliprotein E (ApoE), a plasma glycoprotein, plays a key role in lipoprotein metabolism. The ApoE genotype has three isoforms (alleles) $\varepsilon 2$, $\varepsilon 3 \& \varepsilon 4$. The ApoE $\varepsilon 4$ allele has been associated with the development of Alzheimer's disease (AD), worse outcome after traumatic brain injury as well as ischemic stroke. In this prospective study we investigated if the development of SAH, tendency of cerebrovascular vasospasm, and 1-year neurological outcome are dependent on ApoE polymorphism. Approval was obtained from the University Ethics Committee.

Methods: In the first part of the study 190 patients with SAH from verified intracerebral aneurism/s were consecutively enrolled. The patients' genetical constellation on the ApoE allele was compared to an age and gender matched control group of 211 healthy individuals from the same geographical area.

In the second part of the study 147 patients with aneurismal SAH were genetically scanned for ApoE genotype and followed during treatment and recovery. Tendency to develop early cerebrovascular vasospasm, long term neurological deficit and one year outcome were assessed by using the National Institute of Health Stroke Scale (NIHSS), Bartell index and Extended Glasgow Outcome Scale (GOSE) and were related to the existence or absence of ApoE $\varepsilon 4$ allele.

Results: The incidence of ApoE alleles 2, 3 \& 4 were similar between the patients with $\mathrm{SAH}$ and the age- and sex-matched control group.

We found no significant difference in early cerebrovascular spasm development between SAH patients having $\varepsilon 4$ allele and those who did not. Neither could we detect an influence of ApoE polymorphism on long term neurological deficit nor outcome between these patient groups. This result was independent of the therapy chosen (surgical clipping/endovascular coiling).
Conclusions: Genetic differences, concerning ApoE polymorphism do not seem to influence either the development of SAH nor the recovery and long term neurological outcome, not even when factors like cerebrovascular vasospasm and methods of securing the aneurism are taken into consideration.

\section{G-2}

\section{Circulating levels of endothelin-1 (ET) in patients with acute bacterial meningitis}

K. Møller ${ }^{1}$, G. Strauss ${ }^{2}$, F. Tofteng ${ }^{2}$, C. Sahl ${ }^{3}$, T. Qvist ${ }^{1}$, L. Edvinsson ${ }^{4}$

Departments. of ${ }^{1}$ Infectious Diseases, ${ }^{2}$ Hepatology, and ${ }^{3}$ Intensive Care, University Hospital Rigshospitalet; ${ }^{4}$ Department of Experimental Medicine, University Hospital Glostrup

Introduction: The endogenous vasoconstrictor ET has been implicated in the pathophysiology of bacterial meningitis [1]. We measured arterial plasma concentrations and cerebral net flux of ET in patients with acute bacterial meningitis as compared to healthy controls.

Methods: The study was preceded by Institutional Review Board approval and informed consent from the subjects or their families. We included seven mechanically ventilated adult patients with severe bacterial meningitis and sepsis. Sixteen healthy volunteers (controls) were studied during acute voluntary hyperventilation ( $\mathrm{N}=8), 90$ minutes after an IV endotoxin bolus ( $2 \mathrm{ng} / \mathrm{kg}, \mathrm{N}=8)$, and during norepinephrine infusion $(\mathrm{N}=8)$. Cerebral plasma flow (CPF) was measured by the Kety-Schmidt technique. The plasma concentration of ET was measured in blood samples drawn from arterial and jugular bulb catheters. The cerebral net flux was calculated by multiplication of the arterial to jugular plasma concentration difference and CPF. Statistical analysis was performed using non-parametric testing and $\mathrm{P}<0.05$.

Results: CPF did not differ between groups. The arterial plasma concentration of ET was similar in patients and controls at baseline. In controls, ET plasma levels remained unchanged during hyperventilation, but increased after endotoxin injection $(P=0.01)$ and during norepinephrine infusion $(P=0.02)$. In patients, no net flux of ET was found. By contrast, controls exhibited a cerebral net efflux of ET at baseline $(P=0.04)$, which persisted during hyperventilation $(P=0.04)$ and during norepinephrine infusion $(P=0.04)$, but was abolished after endotoxin injection.

Conclusion: According to this study, plasma levels of ET are similar in patients with severe bacterial meningitis and healthy volunteers. In the latter 
group, ET plasma levels are increased during norepinephrine infusion and after endotoxin injection. The absence of a cerebral efflux of ET-1 in patients with bacterial meningitis may be due to a limited sample size in this group or, since endotoxin injection abolished the efflux in volunteers, to the presence of sepsis.

Reference:

1 Pfister LA et al. Ann Neurol 2000; 47: 329-335.

\section{G-3}

Brain natriuretic peptide (BNP) is a marker of subarachnoid haemorrhage severity

G. Audibert ${ }^{1}$, G. Steinmann ${ }^{1}$, N. de Talance ${ }^{2}$, C. Charpentier ${ }^{1}$,

M.H. Laurens, P.M. Mertes

${ }^{1}$ Department of Anaesthesia and Critical Care, ${ }^{2}$ Renal and Metabolic

Function Exploration Laboratory, Nuclear Medicine Laboratory,

Hopital Central, Nancy, France

Introduction: Hyponatremia is frequently associated with severe aneurysmal subarachnoid haemorrhage (SAH). Proposed mechanisms include cerebral salt wasting syndrome or the syndrome of inappropriate secretion of antidiuretic hormone. Increased blood levels of brain natriuretic peptide (BNP) have been reported [1,2]. However, in most of the studies sodium intake was not controlled. The aim of this study was to assess water and sodium regulation after severe $\mathrm{SAH}$ in patients with large sodium intake.

Methods: During 18 months, 20 patients with severe aneurismal SAH (WFNS

4.5) were prospectively included. Replacement of sodium was at least $4.5 \mathrm{mmol} / \mathrm{kg} / \mathrm{d}$ and adjusted upon urinary measurements. Fluid loading was adjusted according pulse pressure variations. Renal function, and hormones involved in electrolyte and water homeostasis (vasopressin (AVP), renin, angiotensin, aldosterone and natriuretic peptides) were assessed every 3 days for 10 days (period 1, 2 and 3 ). Cardiac function was assessed using EKG, echocardiography and troponin Ic (Tnlc). Outcome was assessed using 3 month G.O.S.

Results: A BNP increase $(p=0.05)$ and hypernatremia occurrence $(p=0.008)$ were associated with an unfavourable outcome. No hyponatremia occurred. Natremia was not correlated neither with AVP, nor aldosterone, nor renin but was correlated with angiotensin $\left(r^{2}=0.48\right)$. BNP was correlated with Tnlc $(p<0.001)$ and with natremia.

Conclusions: After severe SAH, BNP increase is associated with an unfavourable outcome. The hypothesis of a BNP cardiac source is supported by the associated increase in Tnlc.

References:

1 Tomida M et al. Stroke 1998; 29: 1584-1587.

2 Berendes E et al. Lancet 1997; 349: 245-249.

\section{G-4}

Apolipoprotein E (APOE) promoter polymorphism A-491T is associated with increased intracranial pressure and reduced cerebral perfusion pressure in human traumatic brain injury J.C. Goodman ${ }^{1}$, M. Van², S.P. Gopinath ${ }^{2}$, A.B. Valadka², C.S. Robertson ${ }^{2}$ Baylor College of Medicine, Departments of ${ }^{1}$ Pathology and ${ }^{2}$ Neurosurgery (Neuropathology), Houston, TX, USA

Background: Exonic polymorphisms in APOE give rise to epsilon 2 (E2), epsilon 3 (E3), and epsilon (E4) alleles. The E4 allele has been associated with increased risk of Alzheimer's disease (AD) and poor outcome in traumatic brain injury (TBI). Single nucleotide polymorphisms (SNP's) in the promoter region of APOE have functional effects on transcription activity thereby regulating expression levels of E2, E3, and E4. The G-219T promoter SNP has been associated with unfavourable outcome following TBI. The A-491T SNP is associated with increased risk of AD possibly due to increased expression of APOE while the functionally active T-427C polymorphism is not. We examined the effect of A-491T and C-427T on physiological parameters in TBI.

Material and Methods: SNP analysis was performed by PCR using A-491T and $\mathrm{C}-427 \mathrm{~T}$ primers followed by direct sequencing in 42 patients with severe TBI in whom detailed physiological data was available.

Results: The AVT and T/T genotypes of APOE A-491T are associated with higher average ICP $(26 \pm 15 \mathrm{mmHg})$ and peak ICP $(53 \pm 19 \mathrm{mmHg})$ com pared to A/A which had a lower average ICP $(18 \pm 8 \mathrm{mmHg})$ and peak ICP $(38 \pm 17 \mathrm{mmHg})$. The A/T and T/T genotypes of APOE A-491T are associated with lower average CCP $(60 \pm 20 \mathrm{mmHg})$ and nadir CCP $(26 \pm 23 \mathrm{mmHg})$ compared to A/A which had a higher average CCP $(76 \pm 15 \mathrm{mmHg})$ and nadir CCP $(47 \pm 18 \mathrm{mmHg})$. No differences were observed in jugular venous oxygen saturation or brain tissue partial pressure of oxygen. No genotype related differences in ethnicity, age, gender, severity of injury, intensity of therapy, or injury type (diffuse versus focal), delayed haematoma were seen. No significant effects of T-427C were detected.

Conclusions: The A/T and T/T genotypes of APOE A-491T are associated with increased ICP and decreased CPP following TBI, while A/A is associated with lower ICP and higher CPP that may exert a protective effect in TBI. These genotype effects are opposite those seen in Alzheimer's disease where $\mathrm{A} / \mathrm{T}$ and $\mathrm{T} / \mathrm{T}$ are protective, and $\mathrm{A} / \mathrm{A}$ is associated with increased risk. It is possible that increased expression of APOE conferred by A/A is beneficial in the short term in TBI while chronic increased expression places the patient at risk for AD. Up-regulation of APOE is seen following TBI and in this context may play a role in neuronal repair by mediating recycling of damaged membrane components and fostering neurite extension. In addition, and particularly relevant to the present physiological findings, APOE deficient animals have increased cerebral edema following closed injury suggesting that APOE may ameliorate cerebral edema in TBI. Additionally, recent studies suggest that APOE genotype and expression levels may have effects on cerebral blood flow regulation.

The genetic background of a head injured person may influence propensity to complications and pathophysiological responses to injury. Application of the functional genomics of TBI may permit individualized prognostication and tailored monitoring and intervention.

\section{G-5}

Neurones express macrophage inflammatory protein-2 following traumatic brain injury in the rat J.K.J. Rhodes ${ }^{1,2}$, P.J.D. Andrews ${ }^{1}$, J. Sharkey ${ }^{2}$

${ }^{1}$ Western General Hospital, Edinburgh, ${ }^{2}$ Astellas CNS in Edinburgh

Introduction: The expression of inflammatory mediators and the recruitment of leucocytes into the acutely injured brain are implicated in the pathogenesis of secondary brain injury [1]. However the inflammatory response to brain injury, its control and modulation remain incompletely described. The neutrophil chemotactic cytokine macrophage inflammatory protein-2 (MIP-2) is expressed in vitro by glial and cerebral vascular endothelial cells. The in vivo expression of MIP-2 in response to traumatic brain injury has been demonstrated previously. The production of this chemokine by glia was implied but not demonstrated [2]

Methods: We have investigated the cellular localisation of MIP-2 in the lateral fluid percussion model of focal brain injury. In accordance with the Animals (scientific procedures) Act 1986 and after review by the animal procedures committee, anaesthetised male Sprague Dawley rats received a moderate (1.7-2.0 atm) lateral fluid percussion injury. At $0,4,8,12$ \& $24 \mathrm{~h}$ after injury brains were harvested and dissected into anatomical regions. MIP-2 levels in the cortex were analysed by ELISA

Results: After injury MIP-2 was significantly increased in the injured cortex, peaking at $4 \mathrm{~h}$ after injury and declining rapidly to baseline by $12 \mathrm{~h}$. Imminohistochemical staining of coronal sections from $4 \mathrm{~h}$ after recovery with an anti-MIP-2 antibody and the neuronal marker anti-NeuN localised MIP-2 expression to the cytoplasm of shrunken necrotic neurones.

Conclusion: In vitro chemokines are expressed by glial in response to proinflammatory cytokines. We have localised MIP-2 expression following focal brain injury to necrotic neurones. Our in vivo results suggest that chemokine release may also be a fundamental primary response to tissue damage in the brain, initiating neutrophil chemotaxis.

References:

1 Menon DK, Matta BF. Intensive care after acute head injury. In: Matta BF, Menon DK, Turner JM, eds. Textbook of Neuroanaesthesia and Critical Care. London: Greenwhich Medical Media Ltd, 2000: 35-49.

2 Otto VI, Stahel PF, Rancan M, Kariya K, Shohami E, Yatsiv I, Eugster HP, Kossmann T, Trentz O, Morganti-Kossmann MC. Regulation of chemokines and chemokine receptors after experimental closed head injury. Neuroreport 2001; 2059-2064.

\section{G-6}

Slow potential changes sustained by infarcting human brain tissue: significance in the characterisation of spreading depolarisation

R. Bhatia ${ }^{1}$, M. Fabricius ${ }^{2}$, P. Hashemi ${ }^{3}$, S. Fuhr², M.G. Boutelle ${ }^{3}$, M. Lauritzen ${ }^{2}$, A.J. Strong ${ }^{1}$

${ }^{1}$ Department of Clinical Neurosciences, King's College, London, ${ }^{2}$ Department of Neurophysiology, University of Glostrup, Denmark, ${ }^{3}$ Department of Bioengineering, Imperial College, London

Introduction: Spreading depolarisations occurring in human brain tissue after acute focal injury have yet to be fully characterised. A broad classification of 
events into (i) the 'cortical spreading depression (CSD)' type with pre-existing baseline activity and a variable degree of electrical recovery after the depolarisation wave, and (ii) the 'periinfarctive (PID)' type where there is little or no tissue recovery or pre-existing baseline activity, has been suggested by Fabricius et al [1]. Monitoring tissue which is undergoing infarction may give us further insight into the nature of spreading depolarisation in severely compromised brain tissue.

Methods: The research monitoring protocol was approved by the local research ethics committee. A 6 electrode subdural electrocorticography (ECoG) strip was placed onto the left middle frontal gyrus after the evacuation of an acute subdural haematoma in a 51 -year-old female with a history of head trauma 72 hours previously. A CMA 70 microdialysis catheter was inserted into cortex under electrodes 4 and 5 (perfusion rate $2 \mu \mathrm{L} / \mathrm{min}$, using artificial CSF as perfusate); an intraparenchymal intracranial pressure (ICP) transducer was sited nearby. The craniotomy bone flap was not replaced due to brain swelling at the end of the operation. Post-operative monitoring of parameters including mean arterial blood pressure (MAP), 30-second microdialysate glucose and lactate, 4 channel ECoG and ICP began 1 hour post-operative, and continued for 10 hours. The patient was intubated during this time, and sedation maintained with propofol: $150 \mathrm{mcg} / \mathrm{kg} / \mathrm{min}$. Four hours after the start of monitoring a noncontrast CT scan was performed which revealed pronounced herniation of the frontoparietal cortex out of the craniotomy, with haemorrhagic transformation of the brain underlying the craniotomy edge. Two hours before the end of monitoring it was decided to withdraw all active treatment. The patient died 12 hours later

Results: ICP readings ranged between $50-55 \mathrm{mmHg}$, and MAP: $90-105 \mathrm{mmHg}$ during the period of monitoring. Baseline ECoG of the entire recording showed no cortical activity in the 0.5 to $70 \mathrm{~Hz}$ range except isolated bursts of $1.5 \mathrm{~Hz}$ potentials, amplitude $100 \mu \mathrm{V}$ occurring at very long intervals. The first 4 hours of recording revealed stereotyped slow potential changes (SPCs) of 2-3 minutes duration occurring at approximately 12-minute intervals: $80 \%$ of the SPCs spread along the cortex to adjacent electrodes sequentially, $20 \%$ were synchronous at all electrodes. Between hours 5 and 8 the periodicity of the SPCs decreased to 24 minutes. For the final 2 hours of recording, SPCs were less regular (between 25 and 40 minutes interval) but spreading nonetheless. For the latter half of monitoring, microdialysis revealed dialysate glucose $<5 \mu \mathrm{M}$ (plasma glucose $5-8 \mathrm{mM}$ ), and lactate $>3000 \mu \mathrm{M}$ : these values remained unchanged for the remainder of the period of monitoring.

Conclusions: Slow potential changes (SPCs), indicating severe but reversible depolarisations of cortical tissue, are sustainable in severely compromised human brain tissue where there is very little baseline electrical activity. These display a particular periodicity and stereotyped morphology determined by the degree of underlying tissue viability. SPCs are identical to the periinfarctive type (PID) spreading depolarisations previously described. This provides important information on the relevance of spreading depolarisation to the evolution of secondary brain injury.

Reference:

1 Fabricius $\mathrm{M}$ et al. Cortical spreading depression and peri-infarct depolarisation in acutely injured human cerebral cortex. JCBFM 2005; Suppl: Abstract 474

\section{G-7}

The lack of prostacyclin effect in $\mathrm{IP}_{\mathbf{2}}$ receptor deficient mice increases contusion of the traumatised brain

C. Lundblad, P.O. Grände, T. Moska, P. Bentzer

Department of Anaesthesiology and Intensive Care, University and University Hospital of Lund, Sweden

Introduction: It is well known that the endogenous substance prostacyclin has antiaggregatory and vasodilator effects. This means that a normal prostacyclin production may be of importance for maintenance of adequate microcirculation. That prostacyclin is beneficial for microcirculation following brain trauma finds support from 2 previous studies. Thus, it was shown in rat brain that infusion of prostacyclin decreased the cortical lesion following a fluid percussion trauma [1]. In a clinical study using the microdialysis technique it was shown that prostacyclin infusion reduced interstitial lactate and lactate/pyruvate ratio and increased interstitial glucose in the penumbra zone following a head trauma [2], indicating improved microcirculation. The significance of the endogenous prostacyclin production is, however, still unclear. The present study is an attempt to evaluate the importance of the endogenous prostacyclin production for the development of contusion volumes in the traumatised brain.

Methods: The study was performed on the $\mathrm{IP}_{2}$ receptor deficient male mice, thereby lacking the effects of prostacyclin to be compared with litter mates male mice with a functional $\mathrm{IP}_{2}$ receptor and with wild type (C57bl/6) male mice. The anaesthetised mice were exposed to a standardised controlled cortical impact injury causing a significant lesion. The mice brain were fixed, and analysed seven days following the trauma regarding the contusion volume. Results: The relative contusion volume of the traumatised cortical hemisphere of $11.2 \pm 4.2 \%(S D)$ in the $\mathrm{IP}_{2}$ receptor deficient mice $(\mathrm{n}=11)$ was significantly larger than in the wild type mice of $7.6 \pm 3.4 \%(n=10)$ and in the litter mate mice of $6.8 \pm 3.2 \%(n=6)$. The 2 control groups did not differ in between. Conclusions: The endogenous prostacyclin effects are beneficial by reducing the contusion areas of the traumatised brain.

\section{References:}

1 Bentzer $\mathrm{P}$ et al. Infusion of prostacyclin following experimental brain injury in the rat reduces cortical lesion volume. J Neurotrauma 2001; 18: 275-285.

2 Grände PO et al. Low-dose prostacyclin in the treatment of severe brain trauma evaluated with microdialysis and jugular bulb oxygen measurements. Acta Anaesthesiol Scand 2000; 44: 886-894.

\section{G-8}

\section{Lower albumin levels correlate with poor outcome in traumatic brain injury}

F. Bernard ${ }^{1,2}$, J. Outtrim ${ }^{1}$, D.K. Menon ${ }^{1}$, B.F. Matta ${ }^{1}$

${ }^{1}$ Anaesthesia Department, Addenbroke's Hospital, Cambridge, UK, ${ }^{2}$ Intensive Care Department, Hôpital du Sacré-Coeur, Montréal, Canada Introduction: Low albumin level has been correlated with poor outcome in intensive care. Whether albumin in this case is merely a reflection of disease severity or actually plays an active role in organ dysfunction is still a matter of debate but evidence is emerging suggesting causality. Albumin administration can reduce infarct size and improve outcome in stroke patients [1]. We aimed at determining the relation between albumin levels and neurological outcome in TBI patients.

Methods: The local ethics committee approved this study. We retrospectively analysed 113 patients who sustained a TBI. Albumin was measured within 10 days of TBI, at the time as a corticotropin stimulation test was performed due to severe hemodynamic instability. Age, apache II score, ISS and severity of head injury were recorded. All patients were intubated and managed according to a standard protocol to control raised intra cranial pressure. The Glasgow Outcome Score (GOS) was recorded at 6 months. Results: Mean age was $35 \pm 15$ and $79 \%$ were male. $4 \%, 25 \%$ and $70 \%$ had mild, moderate and severe $\mathrm{HI}$ defined by the GCS on admission. Mean apache II and ISS scores were $15 \pm 6$ and $24 \pm 10$. Albumin levels were low for the whole cohort ( $19 \pm 4 \mathrm{~g} / \mathrm{L}$ ). A lower albumin level was associated with a lower GOS: GOS 1 (dead) =18 g/L, GOS 5 (normal) $=20 \mathrm{~g} / \mathrm{L}(\mathrm{p}<0.05$ with spearman rank correlation). GOS 1 had a statistically lower albumin level than group 3, 4 and 5. There is, however, considerable overlap between groups. The multiple linear regression analysis showed that the apache score and albumin levels were related with outcome $\left(r^{2}=0.16, p<0.0001\right)$. Conclusion: Albumin levels are uniformly low in the first ten day after TBI. Results suggest that a lower albumin level is correlated with poorer neurological outcome. It is still undetermined if a causal relationship exists as the apache score was also related to poor outcome. However, in the case of blood brain barrier dysfunction, oncotic pressure becomes an important factor for oedema formation. It is conceptually attractive to think that lower albumin would promote its formation. In fact, albumin administration has been used successfully in the treatment of severe TBI [2]. A randomized controlled trial is needed to resolve this important issue.

References:

1 Dziedzic T. Stroke 2004, p e156-e158.

2 Tomita H. Acta Neurochir Suppl (Wein), p 547-549.

\section{G-9}

Xenon impairs neurocognitive and histologic outcome following cardiopulmonary bypass combined with cerebral air embolism in the rat

B. Jungwirth ${ }^{1}$, M.L. Gordan ${ }^{1}$, M. Blobner ${ }^{1}$, W. Schmehl ${ }^{2}$, E.F. Kochs ${ }^{1}$, G.B. Mackensen

${ }^{1}$ Klinik für Anaesthesiologie, Klinikum rechts der Isar, München, and ${ }^{2} A G A$ Linde Healthcare, Sweden

Introduction: The inert gas xenon has recently been shown to provide neuroprotective properties. This, together with its attractive hemodynamic profile, make xenon an ideal candidate for patients undergoing cardiac surgery. 
However, in vitro studies have demonstrated xenon's disposition to expand gaseous bubbles [1] that during cardiopulmonary bypass (CPB) present as cerebral air emboli (CAE). As CAE have been associated with neurocognitive deficits following cardiac surgery, any amplification of CAE could be detrimental. To elucidate this safety concern, the current study aimed to investigate the impact of xenon on neurologic, cognitive and histologic outcome following CPB combined with $\mathrm{CAE}$ in the rat.

Methods: With IRB approval, 40 anesthetized rats were cannulated for CPB and CAE. Rats in two of four groups were subjected to 90 min of normothermic CPB with 10 equally sized CAE $(0.3 \mu$ l per embolus) injected repetitively via the right internal carotid artery. The other two groups (sham) were exposed to the same surgical and anesthetic regime but not to CPB or CAE. Groups were further subdivided into xenon (56\% xenon, $5 \% \mathrm{~N}_{2}, 34 \% \mathrm{O}_{2}$ $\left.5 \% \mathrm{CO}_{2}\right)$ and nitrogen $\left(61 \% \mathrm{~N}_{2}, 34 \% \mathrm{O}_{2}, 5 \% \mathrm{CO}_{2}\right)$ groups with the accordant mixture inhaled $20 \mathrm{~min}$ prior to, during and $30 \mathrm{~min}$ following $\mathrm{CPB}$. Fine and gross neurologic function as well as cognitive function were tested unti postoperative day 14 when cerebral infarct volumes were determined Neurologic and cognitive parameters were analyzed using ANOVAs while infarct volumes were compared with student t-tests $(p<0.05)$.
Results: All animals undergoing CPB and CAE showed transient deficits in gross neurologic function. Further, animals exposed to xenon during CPB and CAE demonstrated impaired fine motor and cognitive performance that persisted until postoperative day 14. Consistent with this, infarct volumes were larger in the $\mathrm{CPB} / \mathrm{CAE} / \mathrm{xenon}$ group compared to the $\mathrm{CPB} / \mathrm{CAE} /$ nitrogen group ( $83 \pm 16 \mathrm{~mm}^{3}$ versus $33 \pm 19 \mathrm{~mm}^{3} ; \mathrm{p}=0.03$ ).

Conclusions: For the first time this combined rat model of CPB and CAE allowed to study the safety profile of xenon with special regard to its disposition to expand gaseous bubbles in vivo. Although the kinetics of CAE were not studied directly, indirect information about xenon's effect on CAE was generated; animals anesthetized with xenon demonstrated worse functional and histologic outcome following CPB and CAE. We conclude that the potential neuroprotective properties of xenon as recently revealed in a model of CPB in the rat may have been overwhelmed by the effects of xenon on CAE [2].

References:

1 Lockwood G. Expansion of air bubbles in aqueous solutions of nitrous oxide or xenon. Br J Anaesth 2002; 89: 282-286.

$2 \mathrm{Ma} \mathrm{D}$ et al. Xenon attenuates cardiopulmonary bypass-induced neurologic and neurocognitive dysfunction in the rat. Anesthesiology 2003; 98: 690-698.

\section{Group H: Imaging}

\section{$\mathrm{H}-1$}

\section{Coexisting low cerebral blood flow and volume in head injury is due to microvascular ischaemia, not hypometabolism}

J.P. Coles ${ }^{1,3}$, D.K. Menon ${ }^{1,3}$, T.D. Fryer, D.A. Chatfield ${ }^{1,3}$, A.K. Gupta ${ }^{1,3}$, L.A. Steiner ${ }^{1,2,3}$, A.J. Johnston ${ }^{1,3}$, J. Nortje ${ }^{1,3}$, P.J.A. Hutchinson ${ }^{2}$, F.I. Aigbirhio ${ }^{3}$, J.C. Clark $^{3}$, J.D. Pickard ${ }^{2,3}$

Departments of ${ }^{1}$ Anaesthesia and ${ }^{2}$ Neurosurgery, and ${ }^{3}$ Wolfson Brain Imaging Centre, University of Cambridge, Cambridge, UK

We have shown that cerebral blood volume (CBV) is directly, rather than inversely, related to cerebral blood flow (CBF) both at early $(<24$ hours) and late (1-5 days) time points after head injury. These findings suggest microvascular ischaemia, dysautoregulation or metabolically coupled changes in $\mathrm{CBF}$, rather than autoregulatory vasodilatation in response to classical macrovascular ischaemia. It is important to establish whether areas with low $\mathrm{CBF}$ and CBV represent microvascular ischaemia or hypometabolism, since the management of these two physiological scenarios is widely different. We have used high oxygen extraction fraction on triple oxygen positron emission tomography $\left(3 \mathrm{O}_{2} \mathrm{PET}\right)$ to identify true ischaemia, and hence allow us to differentiate between these two conditions.

We undertook $61{ }^{15} \mathrm{O}$-PET studies with measurement of CBF and CBV ten in healthy volunteers and 51 in patients with TBI ( 15 within 24 hours, and 36 at 2-6 days post injury). The median (range) admission Glasgow Coma Score (GCS) in patients was $7(3-13)$, but, at the stage when imaging was undertaken, all patients required sedation and/or ventilatory support for ICP elevation or reductions in GCS to $<8$. Patients received protocol driven therapy aimed at maintaining ICP $<20 \mathrm{mmHg}$, cerebral perfusion pressure (CPP) $>70 \mathrm{mmHg}$, and $\mathrm{PaCO}_{2}$ at $\sim 4.5 \mathrm{kPa}$.

PET studies were undertaken using steady-state techniques. Parametric maps of $\mathrm{CBF}, \mathrm{CBV}, \mathrm{CMRO} 2$ and OEF were calculated using a blood-brain partition coefficient for $\mathrm{H}_{2}{ }^{15} \mathrm{O}$ of 0.95 and a small to large vessel haematocrit ratio of 0.85 . A standardised map with 12 regions of interest (ROI) was constructed for the supratentorial compartment, based on vascular territories and anatomical structures.

CBV was directly, rather than inversely, related to $\mathrm{CBF}$ at both time points (R: 0.56 and 0.48 , respectively; $p<0.0001$ for both). However, both CBF and CBV were inversely related to OEF ( $p<0.0001 ; R: 0.45$ ), with the highest OEF values (in the $60-70 \%$ range) observed in ROls with mean CBF values in the range of $20-25 \mathrm{ml} / 100 \mathrm{ml} / \mathrm{min}$. There were few ROls which showed CBF values less than $20 \mathrm{ml} / 100 \mathrm{ml} / \mathrm{min}$, but these tended to have OEF values that were somewhat lower.

These data suggest that the concordance of low CBF and CBV in head injury represents microvascular ischaemia, rather than coupled metabolism. While the OEF values observed in the hypoperfused ROIs were not critically high, these were large regions of interest, and were likely to have been confounded by partial volume effects. It is likely that the ROls with OEF values in the region of $70 \%$ would have contained foci of critical ischaemia.

\section{$\mathrm{H}-2$}

Metabolic brain changes detected by ${ }^{1} \mathrm{H}-\mathrm{MRSI}$ are relevant to clinical severity and outcome in traumatic brain injury

S. Marino ${ }^{1,2}$, E. Zei $^{3}$, M. Battaglini ${ }^{1}$, C. Vittori ${ }^{3}$, P. Bramanti ${ }^{2}$, A. Federico ${ }^{1}$, N. De Stefano ${ }^{1}$

${ }^{1}$ Dipartimento di Scienze Neurologiche e del Comportamento, Università degli Studi di Siena, Siena, Italy; ${ }^{2}$ Centro Studi Neurolesi, Facoltà di

Medicina e Chirurgia, Università degli Studi di Messina, Messina, Italy; ${ }^{3}$ U.O.

Neuroanestesia e Terapia Intensiva, Azienda Ospedaliera Senese, Siena, Italy

Objective: To assess whether brain metabolic information provided by proton MR spectroscopic imaging $\left({ }^{1} \mathrm{H}-\mathrm{MRSI}\right)$ can correlate to injury severity and disease outcome in patients with traumatic brain injury (TBI).

Background: TBI is a common cause of neurological damage and disability. Conventional MRI indices can provide critical information for TBI patient care, but MRI abnormalities rarely correlate to clinical severity and outcome. Previous single-voxel MR spectroscopy studies have shown that white matter values of $\mathrm{N}$-acetylaspartate (NAA, a marker of axonal integrity) may provide a close correlation with clinical scores.

Design/Methods: We performed ${ }^{1} \mathrm{H}-\mathrm{MRSI}$ examination in 10 patients with TBI ( 3 females and 7 males, mean age $=44.6$ years). In each subject, ${ }^{1} \mathrm{H}$ MRSI data were acquired within 48-72 $\mathrm{h}$ after trauma from a large volume of interest centred on the corpus callosum and including mostly white matter of both hemispheres. Brain levels of NAA, choline (Cho), creatine ( $\mathrm{Cr}$ ) and lactate (La) were assessed in each TBI patient and in a group of demographically matched normal controls (NC). Metabolite values were expressed as ratio to $\mathrm{Cr}$ and were selected exclusively from voxels located outside of the focal traumatic lesion. ${ }^{1} \mathrm{H}-\mathrm{MRSI}$ measures of TBI patients were correlated to those of Glasgow Coma Scale (GCS) and Glasgow Outcome Scale (GOS) at 3 months. Results: Central brain values of NAA/Cr were lower in TBI patients than in $\mathrm{NC}(\mathrm{NAA} / \mathrm{Cr}$ in TBI $=2.26 \pm 0.9$; NAA/Cr in NC $=3.03 \pm 0.3, \mathrm{p}<0.005)$. In contrast, $\mathrm{Cho} / \mathrm{Cr}$ values were higher in TBI patients than in $\mathrm{NC}(\mathrm{Cho} / \mathrm{Cr}$ in $\mathrm{TBI}=2.1 \pm 1.1$; $\mathrm{Cho} / \mathrm{Cr}$ in $\mathrm{NC}=1.4 \pm 0.2, \mathrm{p}=0.005)$. Increased levels of $\mathrm{Lac} / \mathrm{Cr}$ were found in $5 / 10$ patients. In TBI patients, NAA/Cr and $\mathrm{NAA}+\mathrm{Cho} / \mathrm{Cr}$ values correlated closely with GCS $(r=0.83$ and 0.84 , respectively; $p<0.005$ for both). In contrast, values of $\mathrm{La} / \mathrm{Cr}$ were closely correlated to GOS measures $(\mathrm{La} / \mathrm{Cr}, \mathrm{r}=0.86, \mathrm{p}=0.001)$.

Discussion/Conclusions: Whereas high axonal damage may suggest worse clinical status, impaired brain oxidative metabolism may anticipate worse clinical outcome. Thus, results of the present study suggest that metabolic brain changes occurring after trauma are clinically relevant and could be used, in addition to conventional MRI examination, to evaluate injury severity and monitor disease outcome in TBI patients. 


\section{$\mathrm{H}-3$}

Long-term outcome of patients with ganglionic hemorrhage: influence of intraventricular hemorrhage and occlusive hydrocephalus

S. Nagel ${ }^{1}$, H.B. Huttner ${ }^{1}$, M. Köhrmann ${ }^{1}$, C. Berger ${ }^{1}$, D. Georgiadis ${ }^{2}$, S. Schwab ${ }^{1}$

${ }^{1}$ Department of Neurology, University of Heidelberg, Germany, ${ }^{2}$ Department of Neurology, University of Zurich, Switzerland

Introduction: There is only rare data available addressing the long-term outcome of patients with ganglionic hemorrhage suffering from additional intraventricular hemorrhage (IVH) and hydrocephalus thus requiring an external ventricular drainage (EVD). We compared the long-term outcome of patients with ganglionic hemorrhage and those with ganglionic and additional IVH and hydrocephalus.

Methods: A total of 80 patients with supratentorial ganglionic hematomas were analyzed. 40 patients with pure ganglionic hematomas were matched (with regard to gender, age, GCS, hematoma volume and requirement of mechanical ventilation) to 40 patients with ganglionic plus IVH and hydrocephalus requiring an EVD. Outcome analysis was performed using the Barthel Index and the modified Rankin Scale (mRS).

Results: Mean hematoma volume was $25.3( \pm 15.2) \mathrm{cm}^{3}$. Overall longterm outcome was unfavourable (Rankin $4-6$ ) in $65 \%$ of patients. Good outcome $(\mathrm{mRS}<4)$ was observed in $25 / 40(62.5 \%)$ patients with hematoma volume $<25 \mathrm{~cm}^{3}$, as compared to $8 / 40(20 \%)$ patients with hematoma volume $>25 \mathrm{~cm}^{3}$ ( $\mathrm{p}$ chi-squared test $<0.05$ ). The duration of treatment on the intensive care unit was longer for patients with an EVD compared to those with pure ganglionic hematomas (8 [2-19] versus 16 [5-29] days, respectively; Mann-Whitney-U-test $<0.05)$, irrespective of the hematoma volume. In Contrast, the requirement of an EVD did not worsen the long-term outcome of patients with comparable hematoma volumes.

Conclusions: The long-term outcome of patients with supratentorial ganglionic hemorrhage with additional ventricular blood and occlusive hydrocephalus is comparable to that of patients with similar hematoma volumes but no IVH.

\section{H-4}

Brain SG of the non contused areas as a marker of severity in human traumatic brain injury

V. Degos ${ }^{1}$, T. Lescot ${ }^{1}$, A. Zouaoui ${ }^{2}$, H. Hermann ${ }^{1}$, F. Préteux ${ }^{3}$, P. Coriat ${ }^{1}$, L. Puybasset ${ }^{1}$

${ }^{1}$ Anesthesiology Department, ${ }^{2}$ Neuroradiology Department, La Pitié-Salpêtrière Hospital and University of Paris VI, Paris, France, ${ }^{3}$ ARTEMIS Project Unit, Institut National des Télécommunications, Evry, France

Introduction: Radiological attenuation is linearly correlated with Specific Gravity (SG) in human tissue and this property allows to measure in vivo the volume, weight and SG of any tissue by computed tomography. This methodology has been extensively used and validated in the past for lung and heart weight assessment and more recently for the study of SG in traumatic brain injury (TBI) patients [1]. The goal of this study was to assess the link between the initial brain specific gravity (SG) measured non invasively by CT and the clinical symptoms, the therapeutic intensity level and the outcome in human traumatic brain injury.

Methods: Brain weight, volume and SG of the overall intracranial content and of the non contused hemispheric areas were measured retrospectively from initial DICOM CT images (Brainview software) in 120 consecutive patients with severe traumatic brain injury (TBI) occurring between January 2001 and March 2004). Inclusion criteria's were severe TBI requiring mechanical ventilation within the first 24 hours and CT performed at our hospital within the first 24 hours. Control values were obtained from 20 healthy subjects paired for age and intracerebral volume. Clinical characteristics, events that occurred on the scene of the accident and during the ICU period and outcome were extracted from our data bank filled up prospectively. Results: As a mean, the SG of the overall intracranial content and of the non contused hemispheric areas were higher in TBI patients than in controls. Three groups of patients were defined according to the SG of their non contused hemispheric areas: the normal SG group (NSG) ( $n=45$; $38 \%$ ), the moderately increased SG group defined as a SG between 1 and 2 SD above normal (MSG) ( $n=37 ; 31 \%)$ and the highly increased SG group defined as a SG higher than 2 SD above normal (HSG) $(n=38 ; 32 \%)$. The initial GCS decreased as the SG increased (NSG:8.4 \pm 3.3 , MSG:7.6 \pm 3.0 , HSG: $6.6 \pm 3.4, p<0.05)$. The incidence of Marshall's score of 3 or 4 increased with the SG (NSG:16\%, MSG:25\%, HSG:74\%). The occurrence of mydriasis
(NSG:16\%, MSG:22\%, HSG:61\%) and the use of osmotherapy at the scene of the accident (NSG:16\%, MSG:30\%, HSG:60\%) increased significantly with SG (all $p<0.05$ ). The therapeutic intensity level in the ICU increased significantly with SG (incidence of EVD insertion for CSF drainage, barbiturates administration and/or use of hypothermia for refractory increased ICP, $p<0.05)$. At the opposite, the mechanism and the velocity of the accident were similar among the three groups. The outcome at ICU discharge was worse in the HSG group although the difference was not significant anymore at one year.

Conclusion: SG of the non hemispheric areas is increased in human TBI. The initial SG of the non contused hemispheric areas is an indicator of severity in human TBI. The initial SG correlates with clinical (mydriasis) and radiological cerebral swelling (Marshall score), but not to long term prognosis.

Reference:

1 Lescot T et al. A quantitative computed tomography assessment of brain weight, volume and specific gravity in severe head trauma. Int Care Med 2005; in press.

\section{H-5}

\section{Diffusion tensor imaging in chronic head injury survivors}

C.H. Salmond ${ }^{1,4}$, D.K. Menon ${ }^{1,2}$, D.A. Chatfield ${ }^{2}$, J.D. Pickard ${ }^{1,3}$, B.J. Sahakian 4

${ }^{1}$ Wolfson Brain Imaging Centre, University Departments of ${ }^{2}$ Anaesthetics, ${ }^{3}$ Neurosurgery and ${ }^{4}$ Psychiatry, School of Clinical Medicine, University of Cambridge, UK

Introduction: Diffusion tensor imaging (DTI) provides a unique insight into cellular integrity of the brain. Two key indices from DTI are diffusivity (the magnitude of diffusion) and anisotropy (the directionality of diffusion). Whilst conventional magnetic resonance imaging underestimates the extent of pathology following closed head injury, diffusion weighted imaging has been shown to more accurately delineate the extent of cerebral damage.

A number of studies have investigated diffusion weighted imaging in acute head injury cases, reporting decreased anisotropy and abnormal diffusivity. These studies have used region of interest approaches or focussed on global measures of diffusion. There have been only a few case studies of DTI in chronic head injury survivors.

Methods: This study used DTI to investigate structural changes in survivors of head injury at least 6 months after their injury. A voxel-based analysis was carried out on anisotropy and diffusivity maps, in a group of 16 head injury survivors compared with age matched and gender matched controls. The relationship between indices of memory, attention and reaction time and diffusion abnormality was also investigated.

Results: The voxel-based analysis revealed significant bilateral decreases in anisotropy, both in major white matter tracts (such as the corpus callosum and the internal and external capsules) as well as association fibres underlying cortex in the temporal, frontal, parietal and occipital lobes. There were no areas of significant increased anisotropy. The analysis of the diffusivity maps revealed a diffuse, bilateral pattern of abnormality. Statistically significant increases in diffusivity were found in temporal, frontal, occipital and parietal lobes. There were no areas of significant decreased diffusivity. A significant positive correlation was found between diffusivity and the learning and memory index (number of errors on CANTAB paired associate learning) in the left posterior cingulate, left hippocampal formation and left temporal, frontal and occipital cortex.

Conclusions: The nature of the diffusion abnormalities found in this study is consistent with the preliminary case reports of late DTI in head injury survivors. The anatomical locations are considerably more extensive than have previously been reported, presumably due to the greater statistical power of group comparisons.

\section{H-6}

Structural changes in the caudate nucleus in normal pressure hydrocephalus (NPH)

E.E. DeVito ${ }^{1,2}$, C.H. Salmond ${ }^{2,3}$, L.K. Tyler ${ }^{4}$, V. Lupson ${ }^{3}$, B.K. Owler ${ }^{5}$, B.J. Sahakian ${ }^{2}$, J.D. Pickard ${ }^{1,3}$

Departments of ${ }^{1}$ Neurosurgery, ${ }^{2} P$ sychiatry, and the ${ }^{3}$ Wolfson Brain Imaging Centre, University of Cambridge School of Clinical Medicine, Addenbrooke's Hospital, and ${ }^{4}$ Department of Experimental Psychology, University of Cambridge, and ${ }^{5}$ Department of Neurosurgery and Neurology, Institute of Clinical Neurosciences, Royal Prince Alfred Hospital, Sydney

Introduction: Normal pressure hydrocephalus (NPH) is characterised by ventricular dilatation and a clinical symptom triad of gait disturbance, cognitive 
impairment and urinary incontinence. The relationship between ventriculomegaly, abnormal CSF absorption and clinical features remains obscure but recent studies have revealed that local CBF is reduced in the basal ganglia, both at baseline and during modest rises in CSF pressure [1]. We have sought to establish whether this is reflected in reduction in volume of the caudate nucleus and hence might contribute to the cognitive and physical symptomotology of NPH and their amelioration with a CSF shunt.

Methods: Participants included 12 patients with idiopathic NPH and 15 healthy age-matched controls and were recruited with permission from the Local Cambridge Ethics Committee (LREC 03/141; LREC 01/089; LREC 00/069). All participants were scanned on a Bruker Medspec S300 scanner operating at 3.0T using a T1-weighted 3D SPGR sequence with the following parameters: TR $9.7 \mathrm{~ms}$, TE $4 \mathrm{~ms}$, TI $300 \mathrm{~ms}$, flip angle $12^{\circ}$, matrix size $256 \times 256 \times 128$, and field of view $250 \times 250 \times 160 \mathrm{~mm}$. The data sets were linearly spatially normalised and smoothed. The analysis was performed on whole brain images in SPM2 (Wellcome Department of Imaging Neuroscience, London, UK). The results of the VBM analysis informed a separate region of interest $(\mathrm{ROI})$ analysis on bilateral caudate volumes in the same dataset. ROI was performed manually by a single investigator in analyze. Left and right caudate volumes were analysed in separate ANOVAs as percentage of whole brain volume to account for individual variability in brain size (intra-rater reliability: left caudate $(a=0.96)$, right caudate $(a=0.93)$, whole brain $(\mathrm{a}=0.99)$ ).

Results: Both voxel-based morphometry and region of interest methods indicated significant differences in bilateral caudate nuclei between NPH and age-matched controls. VBM: left and right caudate had significantly lower signal intensity in NPH than controls ( $\left.p_{\text {FWEcorr }}<0.05\right)$. ROI: left $(p=0.001)$ and right $(p=0.00006)$ caudate had significantly smaller volumes in the NPH group compared with controls.

Conclusions: Previous studies using ${ }^{15} \mathrm{O}-\mathrm{PET}$ and finite element analysis have indicated that the periventricular tissues are at particular risk with ventricular dilatation. Whether the atrophy of the caudate nucleus is a primary effect of ventriculomegaly or the consequence of damage to its afferent and/or efferent fibers remains to be determined. Damage to this important structure may contribute to some of the cognitive and physical symptoms commonly associated with NPH.

Reference:

1 Owler BK, Momjian S, Czosnyka Z, Czosnyka M, Pena A, Harris NG, Smielewski P, Fryer T, Donovan T, Coles J, Carpenter A, Pickard JD. Normal pressure hydrocephalus and cerebral blood flow: a PET study of baseline values. J Cereb Blood Flow Metab 2004; 24(1): 17-23.

\section{H-7}

\section{The effect of hypertonic saline on cerebral contusion after traumatic brain injury: a tomodensitometric study}

T. Lescot ${ }^{1}$, V. Degos ${ }^{1}$, A. Zouaoui ${ }^{2}$, C. Fetita ${ }^{3}$, J.-C. Muller ${ }^{1}$, F. Preteux ${ }^{3}$, P. Coriat ${ }^{1}$, L. Puybasset ${ }^{1}$

${ }^{1}$ Anesthesiology and ${ }^{2}$ Neuroradiology Departments, Pitié-Salpêtrière Hospital, Paris, France, ${ }^{3}$ Institut Nationale des Télécommunications, Evry, France

Introduction: Hypertonic saline (HS) used at various concentration has been consistently shown to decrease intracranial pressure and cerebra water content in human traumatic brain injury (TBI). However, it could be deleterious in the blood-brain barrier (BBB) disruption areas. The goal of the study was to test this hypothesis by using a regional CT scan quantification of brain weight, volume and specific gravity.

Methods: This study received ethical approval by our local institutional review board and written informed consent was obtained from the patient's next of kin A cerebral CT was performed in 22 patients ( $36 \pm 16$ years) $3 \pm 2$ days after TBI both before and $10 \mathrm{~min}$ after the end of the injection of $40 \mathrm{~mL}$ of $20 \%$ HS (in 20 minutes). CT images were analyzed using a customdesigned software package (BrainView 1.8) specially designed for in vivo quantification of volume, weight and specific gravity of the brain. Two subset of analysis were then performed: the first one on different anatomical segments (hemispheres, brainstem, cerebellum, intraventricular and cisternal cerebrospinal fluid (CSF)) and the second one on contused (CC) and non contused hemispheric tissue (NC). The contusion was defined as abnormal hemispheric appearing tissue on CT images and identified using the thresholding function of Brainview including the hyperdense core of the contusion and the hypodense pericontusional tissue. The volume of each compartment was computed as the number of voxels included in this compartment times the volume of the voxel. The weight of each voxel was computed as its volume times its specific gravity knowing that the specific gravity of a voxel having a radiological density of $0 \mathrm{HU}$ (i.e. water) is $1 \mathrm{~g} / \mathrm{ml}$. The weight of each compartment was computed by summing the weights of all voxels composing it.

Results: HS led to a significant increase in volume of CC (from $67 \pm 56 \mathrm{~mL}$ to $74 \pm 60 \mathrm{~g} ; P<0.001$ ) and decreased in NC (from $1090 \pm 125 \mathrm{~mL}$ to $1077 \pm 122 \mathrm{~mL} ; P<0.001$ ). The effect of HS on weight, volume, and specific gravity of anatomical structures are shown in the Table. Figure shows relation between volume of contused brain and the increase of the contusion's volume after HS infusion.

\begin{tabular}{lccc}
\hline & Baseline & After HS injection & $\boldsymbol{P}$ \\
\hline Hemispheres & & & \\
$\quad$ Weight $(\mathrm{g})$ & $1181 \pm 130$ & $1172 \pm 126$ & $<0.01$ \\
Volume $(\mathrm{mL})$ & $1141 \pm 127$ & $1132 \pm 124$ & $<0.01$ \\
$\quad$ Specific gravity $(\mathrm{g} / \mathrm{mL})$ & $1.0347 \pm 0.0037$ & $1.0350 \pm 0.0035$ & $<0.001$ \\
Brainstem & & & \\
$\quad$ Weight $(\mathrm{g})$ & $28 \pm 6$ & $27 \pm 6$ & 0.2 \\
Volume $(\mathrm{mL})$ & $27 \pm 6$ & $26 \pm 6$ & 0.2 \\
Specific gravity $(\mathrm{g} / \mathrm{mL})$ & $1.0293 \pm 0.0020$ & $1.0296 \pm 0.0022$ & 0.8 \\
Cerebellum & & & \\
Weight $(\mathrm{g})$ & $137 \pm 18$ & $141 \pm 17$ & 0.1 \\
Volume $(\mathrm{mL})$ & $132 \pm 17$ & $136 \pm 21$ & 0.1 \\
Specific gravity $(\mathrm{g} / \mathrm{mL})$ & $1.0369 \pm 0.0040$ & $1.0373 \pm 0.0035$ & $<0.05$ \\
Cerebrospinal fluid & & & \\
$\quad$ Weight $(\mathrm{g})$ & $49 \pm 32$ & $49 \pm 32$ & 0.9 \\
Volume $(\mathrm{mL})$ & $48 \pm 31$ & $48 \pm 31$ & 0.9 \\
Specific gravity $(\mathrm{g} / \mathrm{mL})$ & $1.0180 \pm 0.0061$ & $1.0187 \pm 0.0054$ & 0.1 \\
\hline
\end{tabular}

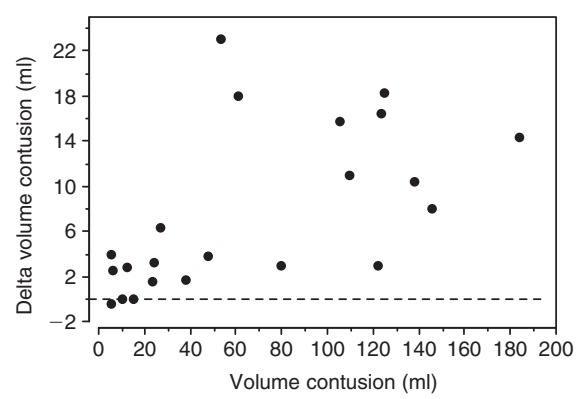

Discussion: HS administration can have deleterious effect on BBB disruption area by increasing the volume of cerebral contusion and should be use with caution in patients who present a great amount of contused brain 


\section{Biographies}

\section{Alastair Buchan}

Professor Alastair Buchan is Professor of Clinical Geratology at the University of Oxford. He works at the John Radcliffe Hospital as an Honorary Consultant Neurologist and heads the Acute Stroke Programme for Oxford. He has recently stepped down from being Head of the Calgary Stroke Programme where, as a Neurologist at the Foothills Medical Centre and Heart and Stroke Foundation Professor of Stroke Research, he led the Calgary Stroke Programme for the last nine years. His laboratory work is concentrated on neuroprotection, tolerance, and preconditioning in experimental ischaemia using models of global and focal ischaemia. More recently, he has investigated the role of a variety of agents which act as neuroprotectants. In 2004, his group was successful at showing that molecular labels could be applied in vivo demonstrating molecular imaging in transient focal ischaemia. If early events can be imaged prior to cell death, this may help develop effective neuroprotection. Clinically, the Calgary Stroke Programme has now treated in excess of 500 people with intravenous thrombolysis and is being guided by the use of high-field imaging in an emergency setting in Calgary. Professor Buchan is now setting up an Acute Stroke Programme in Oxford and hopes to contribute to the new UK Stroke Network for which he is a Strand Leader for Translational Research.

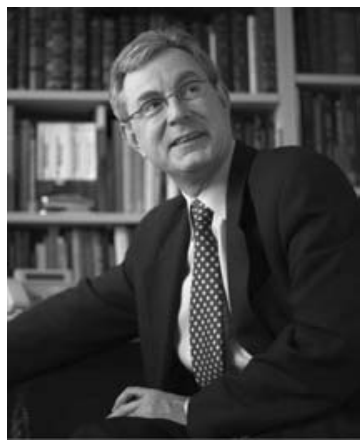

\section{Alexander Baethmann}

Professor Dr. med. Alexander Baethmann, MD, PhD Institute for Surgical Research, Klinikum of the University of Munich, 81366 Munich, Germany. 1960-1966: School of Medicine, Ludwig-Maximilians-University Munich; 1968: Doctoral Degree; 1967-1970: Research Fellow Experimental Neurosurgery, University of Munich; 1970-1972: International Fogarty Fellow of the US National Institutes of Health for a research collaboration with the California Institute of Technology, Pasadena/California; 1974: PhD - Habilitation (Privatdozent) for Experimental Neurosurgery, Ludwig-MaximiliansUniversity Munich; 1979: Assistant; 1983: Associate-Professor of the University of Munich; 1989-1990 and 2002-2005: Acting Director of the Institute for Surgical Research, University of Munich.

\section{Alim L. Benabid}

Professor Alim Louis Benabid born in Grenoble in 1942. He received his MD and his PhD in Physics at Joseph Fourier University of Grenoble (UJFG) and had a sabbatical in basic neurosciences training at the Salk Institute, San Diego. He is currently Professor of Biophysics, Director of the Laboratory of Preclinical Neurosciences at INSERM, Head of Functional Neurosurgery at UJFG Hospital and Coordinator of the Center for Functional Neurosurgery 'Claudio Munari', Milano. He is a Member of the French Academy of Medicine, of the Royal Academy of Medicine in Belgium and of the Academy of Sciences at the Institut de France. His research has initially focused on mathematical modelling of intracranial pressure, MRI spectroscopy, molecular biology of brain tumours and basal ganglia physiology, as well as neurosurgical robotics. Since his discovery, in 1987, of the effects of high frequency stimulation on neural elements, he established the Deep Brain Stimulation therapy for movement disorders, which he extended to several targets and to other pathologies such as dystonia, epilepsy, and experimental models for obesity, psychosurgery. His current research explores the acute and long term biological effects of high frequency stimulation, and its mechanisms of action.

\section{Andreas W. Unterberg}

Chairman and Director, Department of Neurosurgery, Ruprechts-Karls-University of Heidelberg (since 2003), Professor of Neurosurgery (since 1991 at Charité, Humboldt-University of Berlin). Clinical Interests: vascular neurosurgery, pituitary surgery, neurooncology, neurotraumatology. Research Interests: secondary brain damage following trauma, subarachnoid hemorrhage and ischemia (experimental and clinical). 


\section{Anthony Strong}

Professor Strong is Consultant Neurosurgeon and Professor of Neurosurgery at King's College Hospital. The unit was formed in 1995 from the amalgamation of the former Maudsley and Brook Neurosciences Units, producing one of the largest clinical neurosciences services in the UK. Professor Strong's particular clinical interests have been in vascular and skull base neurosurgery, but most recently he has focused largely on a programme of translational brain injury research. In the laboratory, current work concentrates on the haemodynamic effects of peri-infarct depolarisations and the mechanisms governing this. In collaboration with Dr Martyn Boutelle, PhD, the transient changes in lactate and glucose accompanying depolarisation events in both experimental stroke models and in patients with acute brain injury have been characterised, using a rapid-sampling microdialysis method that has undergone continuous refinement since its introduction. In a collaboration with Prof Martin Lauritzen and Dr Martin Fabricius (Glostrup University Hospital, Copenhagen) the group reported, in an expedited publication in Stroke (33: 2739-2744, 2002), the first fully validated description of spreading depression in the human brain, in patients with acute contusional brain injury. The collaboration has now expanded into a multinational effort (COSBID: Cooperative Study of Brain Injury Depolarisations: www.csobid.org), embracing subarachnoid haemorrhage as well as trauma.

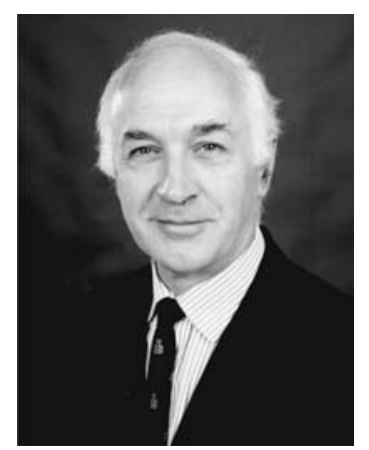

\section{Barbara Jacquelyn Sahakian}

Barbara J. Sahakian is Professor of Clinical Neuropsychology at the Department of Psychiatry, School of Clinical Medicine, University of Cambridge. She obtained her BA (Magna cum Laude) from Mount Holyoke College, her PhD from the University of Cambridge (Darwin College) and her Dip Clin Psych from the British Psychological Society. She held postdoctoral positions at the Massachusetts Institute of Technology (MIT, Cambridge, USA) and the Institute of Neurology (Queen Square, London). Before taking up her academic post at the University of Cambridge, she was a Lecturer and a Senior Lecturer in the Department of Psychiatry, Institute of Psychiatry (London). Her research interests include cognitive psychopharmacology neuropsychology, neuropsychiatry, and neuroimaging. She is probably best known for her work on early detection of Alzheimer's disease, cognitive enhancement using pharmacological treatments and the effects of mania and depression on cognition. She was one of the first researchers to suggest that attentional dysfunction in Alzheimer's disease could be ameliorated using pharmacotherapy such as cholinesterase inhibitors. Professor Sahakian has over 200 publications in scientific journals including Nature, Nature Neuroscience, Science, The Lancet, British Medical Journal, Archives of General Psychiatry, Biological Psychiatry, The Journal of Neuroscience, Brain, Psychopharmacology and Psychological Medicine. Her academic special interests are in communicating science to the general public, women in science and the development of special graduate programmes for integrated research and the enhancement of skills training. Recently, she has contributed to three University of Cambridge initiatives: the establishment of the University of Cambridge-National Institutes of Health (NIH, USA) Health Sciences Scholars Programme; the establishment of the University of Cambridge-Massachusetts Institute of Technology Institute (CMI) MPhil in Biosciences Enterprise; the establishment of the Graduate School of Biological, Medical and Veterinary Sciences. She is also co-inventor of the CANTAB computerised neuropsychological tests, which are in growing use world-wide. Professor Sahakian is a Fellow of Clare Hall, where, until recently, she chaired the Visiting Fellowships and Research Fellowships Committee, and a Bye-Fellow of Christ's College, where she is Director of Studies in Experimental Psychology. In 2004 she was elected as a Fellow of the Academy of Medical Sciences. In 2005 she was awarded the Donders Chair in Psychopharmacology at the University of Utrecht (The Netherlands). From November 2005, she will be on the Committee of Women in Neuroscience for the Society for Neuroscience (SFN, USA).

\section{Bernd Walter Böttiger}

Name: Bernd Walter Böttiger; Degree: MD, DEAA, Full Professor of Anesthesiology; Research Work: 1995-1996 MaxPlanck-Institute for Neurological Research, Cologne, Germany, 1996+1999 Ischemia Research and Education Foundation, San Francisco, CA; Research Awards: 1992 Award of the German and Austrian Society of Internal Intensive Care \& Emergency Medicine, 2000 E.-K.-Frey Award of the German Society of Intensive Care and Emergency Medicine, 2001 KarlThomas-Award of the German Society of Anesthesiology and Intensive Care Medicine, 2001 Research Award of the American Society of Neuro-surgical Anesthesia and Critical Care Medicine.

\section{Claudia Robertson}

Claudia Robertson holds the position of Medical Director of the Neurosurgical Intensive Care Unit at Ben Taub General Hospital and Professor in the Neurosurgery Department at Baylor College of Medicine. Dr Robertson received her M.D. degree from Baylor College of Medicine, and trained in internal medicine at Georgetown University-Washington DC V.A. Hospital, followed by critical care medicine at West Virginia University Hospital. Dr Robertson's research interests include the acute care management of traumatic brain injury and the regulation of cerebral blood flow in neurosurgical disorders. 


\section{Gordon D. Murray}

I completed a mathematics degree at Trinity College Cambridge before going to Glasgow to study for a PhD in Medical Statistics. The PhD project involved collaboration with colleagues at the Department of Neurosurgery at Glasgow, and ever since I have been involved in research projects in head injury and stroke. My main research interests are in clinical trial methodology, which I pursue in collaboration with the European Brain Injury Consortium (EBIC) and its American cousin ABIC. My current position is Professor of Medical Statistics at the University of Edinburgh, where I am based within the Division of Community Health Sciences (www.chs.med.ed.ac.uk).

\section{Howard Yonas}

Howard Yonas, MD, is Director of the Cerebrovascular Programs at the University of New Mexico School of Medicine as well as Chairman of the newly formed Department of Neurological Surgery. Dr Yonas recently joined the faculty at the University of New Mexico coming from the University of Pittsburgh. He joined the faculty at Pittsburgh in 1978 after completing fellowships in microsurgery there and at the Kantonsspital in Zurich, Switzerland. He earned a bachelor's degree in chemistry from the University of Pittsburgh in 1966 and an MD degree from Ohio State University in 1970. After an internship in surgery at the University Hospital of Cleveland, he served two years as a flight surgeon in the United States Air Force. He then completed his residency in neurosurgery at the University Hospital of Cleveland. Dr Yonas is actively involved in research concerning cerebrovascular disease, including the clinical application of quantitative cerebral blood flow measurements, alternate approaches for the physiological monitoring of patients with cerebral ischemia, and better understanding of cerebral aneurysms. Dr Yonas' specialized area of expertise includes extra and intra cranial reconstructive vascular surgery for the prevention of stroke; surgical treatment of intra cranial aneurysms; development of minimally invasive diagnostic approaches to the extra and intra cranial vascular disorders; the measurement of cerebral physiology with a focus on cerebral blood flow for the diagnosis and treatment of cerebrovascular disorders; a focus on trauma directed at improving the level of care for head injured patients with the major focus toward improved physiological monitoring.
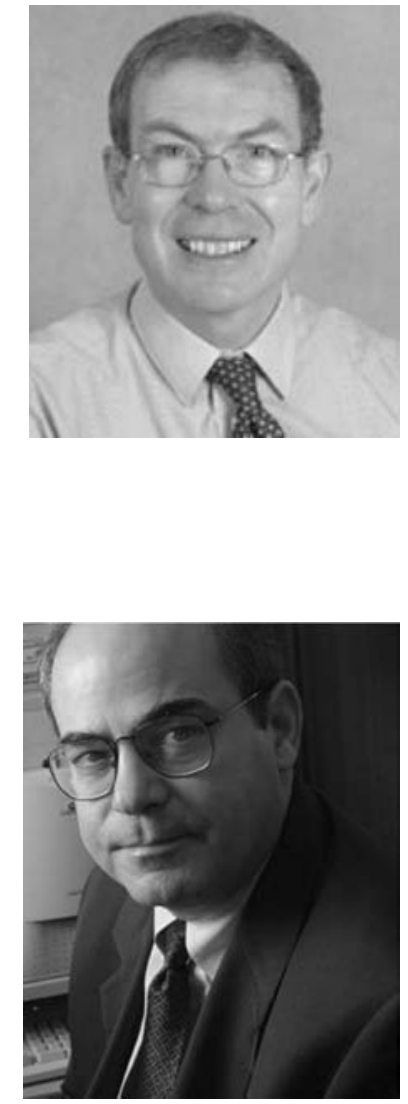

(1)

\section{lan Roberts}

lan Roberts trained as a paediatrician in the UK and then in epidemiology at the University of Auckland, New Zealand and at McGill University, Canada. His research interests include the prevention, treatment, and rehabilitation of injury, with the aim of providing rigorous research evidence on which to base intervention strategies. He is co-ordinating editor of the Cochrane Injuries Group, an international network of individuals who prepare and maintain systematic reviews of the effectiveness of interventions in the prevention, treatment and rehabilitation of injury. He is currently Professor of Epidemiology and Public Health at the London School of Hygiene \& Tropical Medicine.

\section{James A. R. Nicoll}

I am Professor of Neuropathology at the University of Southampton and Consultant Neuropathologist at Southampton General Hospital. Up until 2001 I worked at the Institute of Neurological Sciences, Southern General Hospital, Glasgow. My research interests are mainly in the response of the brain to injury and in neurodegeneration. A particular area of interest is how inter-individual genetic variation may result in different responses to injury and different patterns of disease and what biological mechanisms mediate these differences. Much of the work I will describe here is the result of collaborative multidisciplinary approaches including clinical studies, post mortem neuropathology studies and transgenic animal models undertaken with colleagues in Glasgow and elsewhere.

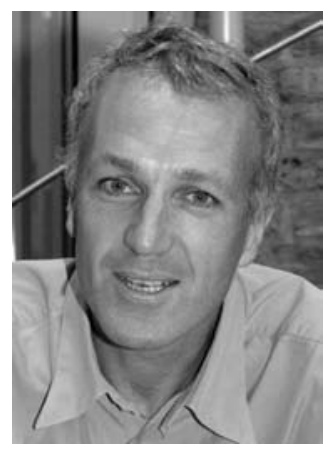




\section{James Fawcett}

James Fawcett is Chairman of the Cambridge University Centre for Brain Repair and Director of Medical Studies at King's College Cambridge. He trained in medicine at Oxford University and St. Thomas Hospital London, then practiced in hospital medicine for four years, latterly in autoimmune disease. His PhD studies were in the development of the visual connections in the nervous system in the laboratory of Mike Gaze at the National Institute for Medical Research in London. Following this he joined the lab of Max Cowan at the Salk Institute. During this time he became increasingly interested in applying developmental principles to the discovery of treatments for the repair of the damaged brain and spinal cord, and when he set up his laboratory at Cambridge University he decided to concentrate on this area. His main interest has been the inhibition of nerve fibre regeneration by scar tissue, and particularly the part played by inhibitory proteoglycan molecules produced by scar tissue in blocking regeneration. Recently he has shown that the same proteoglycan molecules are responsible for turning off brain plasticity during development, and that removing them can reactivate plasticity. He has also worked on brain grafting, brain development, myelination and stem cells.

\section{Jean-Claude Baron}

Jean-Claude Baron, MD, is Professor of Stroke Medicine in the Department of Clinical Neurosciences, University of Cambridge, UK and Honorary Neurology Consultant at Addenbrooke's Hospital. Prior to this appointment, he was Director of Research at the INSERM, Head of the INSERM Neuroscience Unit and Scientific Director of the Positron Emission Tomography laboratory at the School of Clinical Medicine, University of Caen, France. His main area of expertise is the pathophysiology of acute stroke and the mechanisms of recovery, and brain imaging with PET and MR is his main scientific technique. He was elected Fellow of the Royal College of Physicians in 2002 and Fellow the Academy of Medical Sciences in 2003. In 2005 he was awarded the Johannes Wepfer Award of the European Stroke Conference. He is Imaging Section Editor for Stroke, and European Editor of the Journal of Neuroimaging. He is on the Editorial Board of Neurolmage, Cerebrovascular Diseases, European Journal of Neurology, Journal of Cerebral Blood Flow and Metabolism and Clinical Neurophysiology, and was previously on the board of Brain and Journal of Neurology, Neurosurgery and Psychiatry.

\section{John M. Shneerson}

John Shneerson is the Director of the Respiratory Support and Sleep Centre at Papworth Hospital, Cambridge. This is one of the largest providers of non invasive ventilatory support in Europe with over 800 patients in the community on ventilators and 3000 on nasal CPAP for sleep apnoeas. He has a special interest in the physiology and treatment of respiratory failure in neuromuscular disorders and has been a member of several national and international committees concerned with the provision of care for patients in chronic respiratory failure. He is the author of over 300 publications, including a comprehensive text book on respiratory failure, Disorders of Ventilation. He has developed a multidisciplinary weaning programme at Papworth Hospital for patients with neurological and other disorders who have failed to wean from ventilators with conventional methods. He also has a special interest in sleep disorders in neurological conditions.

\section{Jonathan P. Coles}

Jonathan P. Coles is an Academy of Medical Sciences/Health Foundation Clinician Scientist and Honorary Specialist Registrar at Addenbrooke's Hospital, Cambridge, UK. He qualified from Leicester University and trained in Anaesthesia and Intensive Care Medicine in Leicester, Norwich and Cambridge within the United Kingdom. As a clinician specialising within Neurointensive Care and Neuroanaesthesia his research interests are focused on describing the pathophysiology of head injury and optimising patient outcome. Using the combined facilities of the Wolfson Brain Imaging Centre and Neurosciences Critical Care Unit at Addenbrooke's Hospital, patients undergo structural and functional imaging studies using positron emission tomography and magnetic resonance imaging. He has used such imaging tools to define the incidence, temporal evolution and impact on outcome of cerebral ischaemia following head injury. Recent work has focused on the impact of clinical interventions aimed at controlling intracranial pressure such as hyperventilation and elevation of cerebral perfusion pressure. 
Jonathan Gillard is a Reader in Neuroradiology in the University of Cambridge, an Honorary Consultant Neuroradiologist at Addenbrooke's Hospital and a Fellow of Christ's College Cambridge. He undertook his undergraduate training in Guy's Hospital London, graduating in 1988, where he also gained a BSc in Radiological Sciences in 1985 focusing on the use of transcranial ultrasound. His basic radiological training was undertaken in Cambridge; his specialist neuroradiological training was done at Johns Hopkins Hospital and University in Baltimore. His 1997 MD was based on spectroscopic imaging in acute stroke. He now leads active research groups using MR methodologies in atheroma imaging, cerebrovascular imaging and neuro-oncology. He has longstanding experience with CT perfusion imaging as well as the application of new PET imaging tools. Further areas of research include health economics and stem cell treatment in neurodegenerative disease in man.

\section{Juan Sahuquillo}

Juan Sahuquillo works as a neurosurgeon at the Vall d'Hebron University Hospital in Barcelona, Spain. He studied medicine at the University of Barcelona obtaining his medical degree in 1979. He trained as a neurosurgeon at the Neurosurgical Department of the Vall d'Hebron University Hospital. He defended his PhD thesis, entitled Diffuse axonal injury after severe head trauma in 1985. Currently, he is the acting head of Department of the Neurosurgery Department and Associate Professor at the Department of Surgery of the Universidad Autónoma in Barcelona. He is also the scientific coordinator of the neurotraumatology research unit, a multidisciplinary unit in which neurosurgeons, intensive care specialists, neurologists, neurorehabilitators and anesthesiologists collaborate in conducting basic and clinical research in traumatic brain injury and massive middle cerebral artery stroke. He is a member of the Executive Committee of the European Brain Injury Consortium and of the Steering Committee of the Brain IT network. Juan Sahuquillo has participated as principal investigator in various multicenter multinational phase III clinical trials of neuroprotective drugs in severe head injury (lazaroids, nimodipine, dexannabinol etc.). He has obtained national and international grants for conducting research in neurotraumatology. His current main lines of research are normal pressure hydrocephalus, metabolic monitoring and transport of oxygen in the injured brain and neuroinflammation. Currently he is the principal investigator of the IntraCool clinical trial, a pilot clinical trial to determine the feasibility and efficacy of intravascular cooling methods to control high ICP refractory to first line therapeutic measures. In the last two years his unit has begun to study mediators of neuroinflammation induced after traumatic brain injury and stroke by using high cutoff microdialysis membranes.

\section{Kees H. Polderman}

Kees $\mathrm{H}$. Polderman works as a senior consultant in intensive care medicine at the VU university medical center in Amsterdam. He attended primary school at St. George international school in Rome, Italy and grammar school in Hilversum, The Netherlands. He studied medicine at the university of Amsterdam, and trained as an internist and subsequently as an intensivist at the VU university hospital. He obtained the European diploma in intensive care in 2000 . His $\mathrm{PhD}$ thesis entitled Interactions of endocrine systems with cardiovascular significance was completed in 1993 . He also holds the ECFMG (US medical license for foreign medical graduates) and a Bachelor degree in Law. He is a member of the national guideline committee, the scientific committee and of the executive committee of the Dutch Society of Intensive Care, a member of the editorial board of the Netherlands Journal of Critical Care, and of the advisory board of the journal Intensive Care Medicine. His current main lines of research are quality assessment and monitoring in the ICU, metabolic disorders in critically ill patients (especially those with neurological injuries), endocrine dysfunction in critical illness, and the use of therapeutic hypothermia for neuroprotection and cardioprotection. $\mathrm{He}$ is a member of the international steering group of the Northern Hypothermia Network.

\section{Leif Østergaard}

Professor of Experimental Neuroradiology, Leif Østergaard, MD, MSc, PhD, DMed is head of the Neuroradiology Research Unit at Aarhus Hospital and head of the Danish National Research Foundation's Center for Functionally Integrative Neuroscience (CFIN), which is a cross disciplinary brain research center under the Clinical Institute at the University of Aarhus. The center does both basic research - trying to map out the human brain and how it reacts and adjusts to effects of the surrounding physical and social environment - and also medically directed research, trying to find new methods to improved diagnosis and treatment of different neurological diseases, like Parkinson's disease, dementia, stroke and depression. The special research area of Leif Østergaard is Functional Haemodynamics. The functional haemodynamics projects fall into a methodological section, applying basic physics, mathematical modeling and image processing in optimizing techniques to study cerebral haemodynamics by dynamic susceptibility contrast MRI. In a parallel effort, these techniques are applied in identifying mechanisms that integrate cerebral haemodynamics and neuroenergetics. The research of Leif Østergaard has resulted in new brain imaging techniques which are now used worldwide, especially in connection to treatment of stroke patients. 


\section{Lindsay Wilson}

Lindsay Wilson is a neuropsychologist based in the Department of Psychology at the University of Stirling. He first worked with neurological patients as a postdoctoral researcher in the Max Planck Institute for Psychiatry in Munich. Since the 1980s he has collaborated with colleagues at the Institute of Neurological Sciences in Glasgow on studies of head injury and stroke. His research includes work on neuroimaging and neuropsychological function after head injury, and quality of life after brain injury. He has also been involved in developing structured assessments of functional outcome based on the Glasgow Outcome Scale, that have been used in a number of recent clinical trials including STICH, CRASH, and the Dexanabinol trial. He is currently involved in outcome assessment for CONSCIOUS-1, a trial of clazosentan in SAH.

\section{Louis Puybasset}

L. Puybasset MD, PhD, Anesthesiologist and Intensivist. Head of the Neurocritical Care and of the Neuroanesthesia. Anesthesiology Department, Pitié-Salpêtrière Hospital, Paris, France.

\section{Luc De Baerdemaeker}

Staff member Department of Anaesthesiology, University Hospital Ghent, Belgium. I received my title of anesthesiologist in 1997 after 5 years of residency under Prof. Dr G. Rolly at the University Hospital of Ghent, Belgium. In 1997, I received the European diploma in anaesthesiology and intensive care of the European Academy of Anaesthesiology. As a staff member, I started my activities on orthopedic and plastic surgery. After two years I moved to the field of neuroanesthesia and thoracovascular surgery. Under the supervision of Prof. Dr J. Van Aken I have participated in research projects such as $\mathrm{CO}_{2}$ reactivity and CBF measurements using the hydrogen clearance technique and cardiovascular changes during endoscopic third ventriculostomy. My main activity at this moment is in abdominal surgery since I am preparing a PhD on anaesthesia for the morbidly obese.

\section{Luzius A. Steiner}

Luzius A. Steiner was born in Basel, Switzerland in 1966. From 1985 to 1991 he studied at the Medical School, University of Basel, Switzerland and received the MD degree in 1992 from the same University. After a research fellowship in Anaesthesia at the Massachusetts General Hospital, Harvard University, Boston, USA he trained in internal medicine, anaesthesia and critical care medicine in various Swiss Hospitals. In 2000 he started working as a Clinical Research Fellow on the Neuro Critical Care Unit, the Wolfson Brain Imaging Centre and Academic Neurosurgery at Addenbrooke's Hospital in Cambridge, UK under the supervision of Prof. D. K. Menon, Prof. J. D. Pickard, and Dr M. Czosnyka. He received a PhD from the University of Cambridge in 2003 for his research on cerebrovascular reactivity after head injury. Since 2004 he is a consultant at the Department of Anaesthesia, University Hospital Basel, Switzerland and is currently working on the Surgical Intensive Care Unit. He is a Privatdozent in Anaesthesia and Intensive Care Medicine at the University of Basel, Switzerland. His research interests are cerebral perfusion in head injured and other critically ill patients, and perioperative cognitive function.

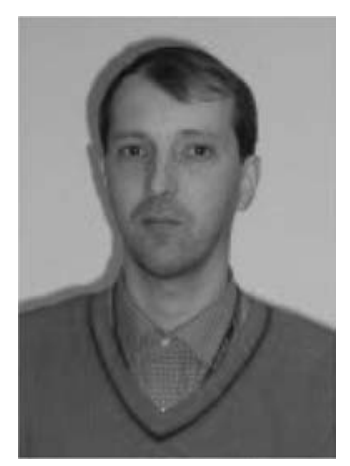




\section{Marek Czosnyka}

Marek Czosnyka, PhD (Warsaw), DSc (Warsaw) in Biomedical Engineering, is Reader in Brain Physics (2003) and Director of Neurosurgical Physics in University of Cambridge Department of Clinical Neurosciences, Neurosurgical Unit. He is also Associate Professor at Warsaw University of Technology, Faculty of Electronics, Warsaw, Poland. Area of research: Pathophysiology of Intracranial Pressure on other brain pressures; Cerebral Blood Flow and its regulation (head trauma, stroke, hydrocephalus, subarachnoid hemorrhage, experimental models); Cerebrospinal Fluid dynamics (hydrocephalus, $\mathrm{BIH}$ ); multi-modal bedside monitoring in neuro-intensive care (head trauma, poor grade SAH, stroke); mathematical modeling of cerebrospinal dynamics; asymmetry of cerebral blood flow, its regulation and cerebrospinal dynamics. $\mathrm{He}$ is an author or co-author of 150 scientific papers listed on PubMed and Science Citation Index.
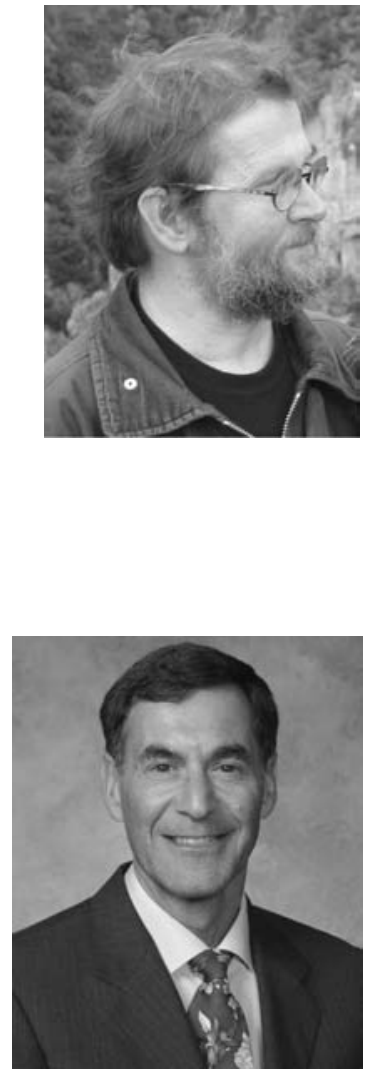
I trained in Internal Medicine initially in Cape Town, and thereafter at Royal Free Hospital in London. In 1976 I undertook a three year Postdoctoral Research Fellowship in Membrane Pathobiology within the Department of Gastroenterology at Stanford University in California after which I trained in Anesthesiology, Pain Management and Critical Care Medicine. I joined the faculty at Stanford in 1981 and was funded by both the National Institutes of Health and the Department of Veterans Affairs to investigate the mechanisms of anaesthetic and analgesic action at the molecular and neural substrate levels. I was initially promoted to a tenured position at Stanford in 1987 and became a full Professor at the same institution in 1993. I was recruited back to the UK in 1999 to head the Department of Anaesthetics and Intensive Care at Imperial College, London. I have received Fellowships from the Royal College of Physicians, the Royal College of Anaesthetists, and the Academy of Medical Sciences. I received the 2003 Award from the American Society of Anesthesiology for Excellence in Research.

\section{Michael N. Diringer}

Michael N. Diringer, MD, received his medical degree from the University of Kentucky in Lexington, Kentucky, completed training in internal medicine and neurology at the State University of New York at Stony Brook, in Stony Brook, New York, and a fellowship in neurosciences critical care at The Johns Hopkins University in Baltimore, Maryland. Dr Diringer is currently Professor of Neurology, Neurological Surgery, Anesthesiology, and Occupational Therapy at Washington University School of Medicine, in St. Louis and serves as the Director of the Neurology/Neurosurgery Intensive Care Unit at BarnesJewish Hospital at Washington University Medical Center. Dr Diringer is a Fellow of the American College of Critical Care Medicine and the Stroke Council of the American Heart Association, has won numbers of awards and has twice been listed in Best Doctors in America. Dr Diringer is funded by the NIH to perform physiological studies testing the impact of treatments on acutely ill neurologic patients using PET and MRI. He is the author of over 100 journal articles, editorials and book chapters. Dr Diringer is a member of the editorial boards of The Neurologist, Stroke Interventionalist, and Neurocritical Care. He is an ad hoc reviewer for the Journal of Neurosurgery, Neurosurgery, Brain, Circulation, Neurology, Stroke, and Critical Care Medicine, among others.

\section{Michael Todd}

I was raised in Phoenix Arizona, but left at age 18 to attend the University of Chicago. My original interest was in basic cell biology, but gradually became more interested in medical science. After a short trip to Denver, Colorado, I finished medical school and did my internship at the University of Chicago. I then spent the next 3 years as a resident - and then Chief Resident - at the Massachusetts General Hospital before moving to San Diego for my Neuroanesthesia fellowship. My fellowship was about 2 years long - and was spent entirely in the laboratory - with people like Harvey Shapiro, John Drummond and Mark Zornow. However, after 7 years - and with a 3-year-old son in hand, we decided that the midwest was a better environment than sunny southern California - and moved to the University of lowa where l've been since. No regrets!
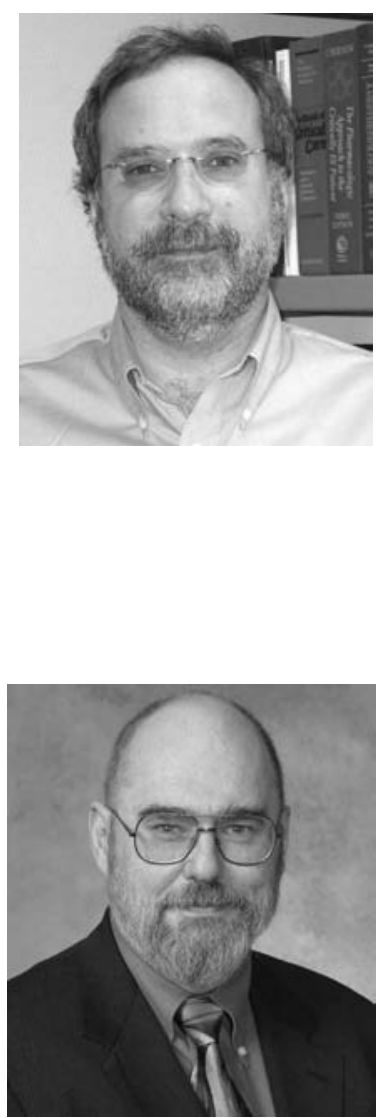


\section{Michael A. De Georgia}

Dr Michael De Georgia was born in Canton, Ohio. He attended the Northeastern Ohio Universities College of Medicine, graduating with honors after completing an accelerated combined 6-year BS/MD program in 1989. Dr De Georgia went on to complete an internal medicine residency at the University of Michigan Medical Center in Ann Arbor, a neurology residency at the Tufts University-New England Medical Center in Boston and a Neurocritical Care fellowship at the University of Heidelberg in Germany. In 1997, Dr De Georgia became the Director of the Neurocritical Care Program and the University Stroke Program at the State University of New York at Syracuse. He joined The Cleveland Clinic Foundation in 1999 and in 2001 became the Head of the Neurological Intensive Care Program and Director of the Neurology/Neurosurgery Intensive Care Unit. Dr De Georgia's interests include the critical care management of ischemic stroke and intracerebral hemorrhage. His research interests are in the use of therapeutic hypothermia following stroke and multi-modal monitoring in the intensive care unit. He has been the Principle Investigator or Co-investigator of multiple national and international trials.

\section{Michael (Monty) Mythen}

Portex Professor of Anaesthesia and Critical Care, Head of The Portex Anaesthesia, Intensive Care and Respiratory Unit at The Institute of Child Health, University College London; Director, The Centre for Anaesthesia, Royal Free and University College London Medical School; Co-Director of The Surgical Outcomes Research Centre, University College London Hospitals; Honorary Consultant Anaesthesia and Intensive Care, University College London Hospitals. Elected Council Member of The Intensive Care Society.

\section{Nicola Latronico}

Born in Stigliano (Italy) on November 15th, 1956; high school diploma completed in Florence and Potenza; medical degree at the University of Pavia in 1982; specialized in Anesthesia and Intensive Care Medicine at the University of Pavia in 1986; second specialization in Neuropathology at the University of Verona in 1992; consultant in Anesthesia and Intensive Care Medicine at the University of Brescia from 1986 to 1997; clinical research physician from 1997 to 2004; associate professor since 2004. Major fields of interest are the Neurosciences, in particular Neurocritical Care and Neurotrauma. Other fields are Multiple Organ Dysfunction Syndrome, Ethics and Evidence-Based Medicine. National coordinator of the Italian study group on Neuroanesthesia and Neurocritical Care since 2000. Board of Directors of the GiViTI (Gruppo Italiano di Valutazione degli Interventi in Terapia Intensiva), the major Italian network of intensive care units, since 1999. Co-founder of the interuniversity research center 'Thomas C. Chalmers' for the methodology of systematic reviews and promotion of the Evidence-Based Medicine. Married, with two children, hobbies: photography, cycling and football.

\section{Nigel Huggins}

Director of Neuroanaesthesia and Consultant Neurointensivist at the Queen Elizabeth Neuroscience Centre in Birmingham. He has been a Committee member of the UK Society for Intravenous Anaesthesia (SIVA UK) since its inception and has presented Internationally on aspects of Intravenous Anaesthesia and on the theory and practise of Remifentanil anaesthesia. His personal portfolio of Neuroanaesthesia cases using Remifentanil exceeds 2500 anaesthetics and therefore places him at the very forefront of the use of Remifentanil in Neuroanaesthesia. He will highlight the desirable attributes of Remifentanil for Neuroanaesthesia and emphasise the clinical advantages of the use of the drug compared to other pharmacological techniques. He emphasises two very important attributes of Remifentanil thus: Firstly 'Remifentanil is a very potent hypnotic sparing agent and this fact surpasses its analgesic properties in importance.' Secondly 'Remifentanil can be likened to an intravenous epidural; blocking out essentially all afferent nociceptive input. This allows Anaesthetists who would be infrequent practitioners of epidural analgesia to confer the benefits of this technique to their patients without the risks associated with epidural drug placement.' In his spare time, Nigel Huggins enjoys horticulture and his smallholding, fine dining, and, not surprisingly, international travel!

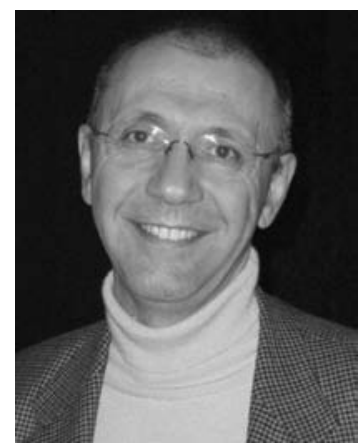




\section{Paul Vespa}

Associate Professor of Neurosurgery and Neurology, David Geffen School of Medicine at UCLA, Director of Neurotrauma Clinic. Education: Post Graduate Fellowship: Neurocritical Care and Clinical Neurophysiology (July 1994-June 1996), University of California, Los Angeles, Residency: Neurology (July 1991-June 1994), University of Virginia, Internship: Riverside Methodist Hospital, Columbus, Ohio (OSU affiliate), Graduate: The Ohio State University College of Medicine (September 1986-June 1990). Ongoing Research: Traumatic Brain Injury: NINDS Program Grant on Brain Metabolism and Neurochemistry, MRI and MR Spectroscopy in Coma and Brain Injury, Post Traumatic Epilepsy NINDS Study, Cerebral Microdialysis Study, Stereotactic Evacuation of Brain Hemorrhage Study, Heme Surgery Study, The Study of the clinical utility of normothermia in the ICU.

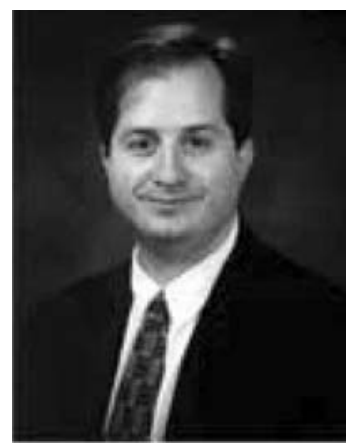

\section{Per-Olof Grände}

Per-Olof Grände, born 1945, is professor in Anaesthesia and Intensive Care at the Lund University and Lund University Hospital of Sweden. He has a basic research education in circulatory physiology and his preclinical research mainly aimed at evaluating normal control mechanisms for perfusion and transcapillary fluid exchange of a tissue. He became specialist in Anaesthesia and Intensive Care in 1986. After that his research became more clinically oriented, concentrating on physiological and pathophysiological aspects of diseases in the intensive care. In this respect he has worked on issues related to increased capillary permeability, such as in sepsis/SIRS and following trauma, and to find measures optimising microcirculation of injured tissues. One important field of research has been to clarify beneficial permeability and microcirculatory effects of prostacyclin and nitric oxide. Another field of research has been to find better treatments of severe head injured patients using basal physiological principles of brain volume and perfusion as guiding tools. This research has resulted in the comprehensive intensive care guidelines for treatment of severe head injury introduced in 1993 denoted the 'Lund Concept'.

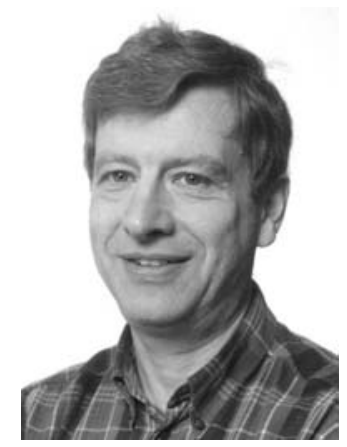

\section{Peter Hutchinson}

Peter Hutchinson, BSc (hons), MBBS, PhD, FRCS (Surg Neurol), holds an Academy of Medical Sciences/Health Foundation Senior Surgical Scientist Fellowship within the Department of Clinical Neurosciences, University of Cambridge and Honorary Consultant Neurosurgeon post at Addenbrooke's Hospital. He has a general neurosurgical practice with a subspecialist interest in the management of trauma and brain tumours. He has a research interest in traumatic brain injury, multimodality monitoring in neuro-critical care and functional imaging. He is also Director of Clinical Studies at Robinson College, an ATLS instructor and medical officer for the British Grand Prix.

\section{Peter John Dawson Andrews}

Reader in Anaesthetics \& Intensive Care, University of Edinburgh, \& Lead Clinician, Critical Care Services, Western General Hospital, Edinburgh. Major Research Interests: (1) SIGNET Trial for the Scottish Intensive Care Society (ISRCTN 87144826); (2) brain cooling research; (3) Project License holder. Lateral Fluid Percussion Model. Peer reviewed Publications: (1) Battison C, Andrews PJD, Graham C, Petty T. Critical Care Medicine 2005; 33(1): 196-202. (2) Howells T, Elf K, jones P, RonneEngström E, Piper I, Nilsson P, Andrews P, Enblad P. Journal of Neurosurgery 2005; 102: 311-317. (3) Andrews PJD, Harris B, Murray GD. British Jounral of Anaesthesia 2005; 94(3): 330-335. (4) Andrews PJD. Intensive Care Medicine 2005; 31(3): 323-324. Associate Editor of Intensive Care Medicine; Section Head European Society of Intensive Care Medicine; Neurological Intensive Care, Emergency Medicine Medical Research Council (UK) College of Experts.

\section{Peter Kirkpatrick}

Peter Kirkpatrick is a Consultant Neurosurgeon based in Cambridge with specialist interests in neurovascular and neurotrauma. His research is clinically based, calling upon a relatively large clinical work devoted to stroke prevention, subarachnoid haemorrhage, cerebral revascularisation, and all aspects of neurotrauma. Novel ways to control raised ICP and provide neuroprotection based upon neurophysiological and metabolic end points is his current line of attack. 


\section{Pol Hans}

University Department of Anesthesia \& Intensive Care Medicine, Liege University Hospital. Diplomate of Anesthesiology and Intensive Care Medicine, University of Liege (1979); Doctorate of Clinical Sciences (1983); Associated Head in the Department of Anesthesia \& Intensive Care Medicine of the Liege University Hospital (1989-current); Head of the University Department of Anesthesia \& Intensive Care Medicine of the Citadelle Hospital. Member of the Belgian Society of Anesthesia and Resuscitation of Belgium, the European Society of Intensive Care Medicine, the Association de Neuro-anesthésieréanimation de Langue Française, the Neuroanaesthesia Society of Great Britain and Ireland, the Society of Neurosurgical Anesthesia and Critical Care, the Society for Intra-Venous Anesthesia, the American Society of Anesthesiologists. Cofounding member and member of the International Society for Neuroemergencies. Member of the European Society of Anaesthesiologists; ESA Council Member (1998-1999), ESA Board (1999-2002) and ESA acting treasurer (1999-2002). Editorial Advisory Board Representative for the Current Anaesthesia and Critical Care. Clinical Occupancies: Anesthesia and perioperative care of neurosurgical, gynecological, obstetrical and cardiovascular surgery patients. Research interests: Neuroanaesthesia, depth of anesthesia monitoring and mechanisms of general anesthesia-induced loss of consciousness, muscle relaxants, free radicals. Home page: www.ulg.ac.be/anesrea

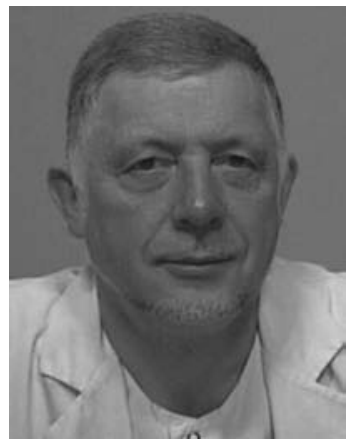

\section{Robert Tasker}

Robert Tasker undertook training in Paediatric Critical Care at Great Ormond Street Hospital, London, and The Johns Hopkins Hospital, Baltimore. He was worked as a full-time Consultant at Great Ormond Street Hospital for before joining the Department of Paediatrics, Cambridge University, in June 1998. Robert Tasker has written extensively on paediatric neurocritical care (e.g., head injury, shaken baby syndrome, non-traumatic coma, status and epilepticus). His current research interests are in the area of Paediatric Neuroscience and Neurocritical Care. In the 1990s he 'cut his teeth' on excitatory amino acid neurotoxicity and the hippocampus, and he has now returned to this area in work on late outcomes after head injury in childhood. In EuroNeuro 2005 he will be presenting recent data gained from collaborative of all children in the UK, in one year, suffering severe head injury.

\section{Stefan Schwab}

Department of Neurology, University of Heidelberg, Heidelberg. Main Interest: Cerebrovascular disorders, neurocritical care, experimental stroke-research, neuroregeneration, neuromuscular disorders, neurooncology. Publications (1/01/2005): 135 peer reviewed papers, 25 review articles, 19 book chapters, 4 books (editor). Awards: 1996, 1st Prize of the 3rd World Conference on Stroke; 1998, 'Hugo Spatz Award' for excellence in stroke research, German Neurological Association (DGN); 1998-2004, 10 Poster awards on several national and international conferences; 2004, 'H.G. Mertens Award' for excellence in neurocritical care research, German Neurological Association (DGN).

\section{Thomas A. D. Cadoux-Hudson}

Degrees and Diplomas: Undergraduate Degree: MBBS(Lond), 1982; Postgraduate Degree: Oxford, New College; 1987-1990: DPhil, 1990; Present Post: NHS post; Consultant, Neurosurgery; Radcliffe Infrimary, Oxford Clinical Lead Dept of Neurosurgery; Program Director: Neurosurgery Regional Training, Medical Student Teaching (part of Neuroscience attachment).
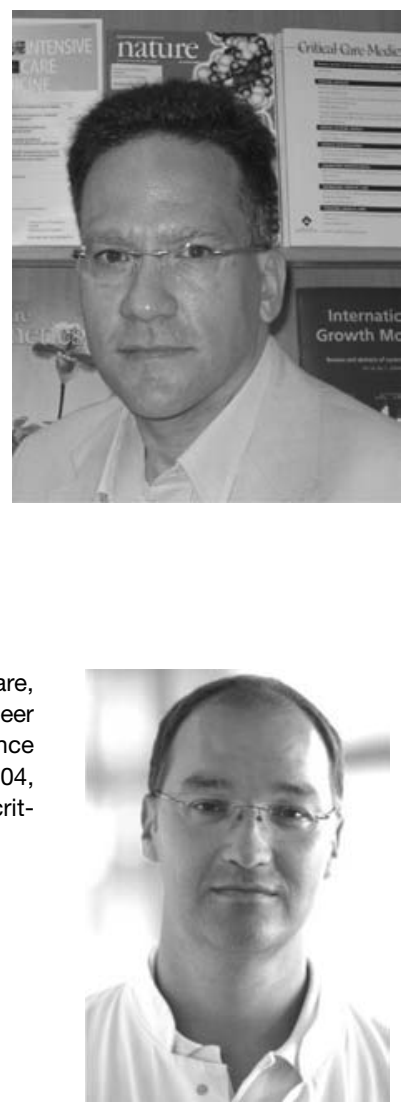


\section{Thomas A. Neff}

Dr Thomas A. Neff received his MD degree from the University of Zurich, Switzerland, in 1992. He did his thesis on the Conventional management of the acute respiratory distress syndrome (ARDS) at the Division of Surgical Intensive Care. During his medical training in Anesthesiology and Intensive Care he published several studies with a special focus on ARDS and volume therapy in traumatic brain injury (TBI). In 2001, Dr Neff received a research grant from the University of Zurich, and he pursued a three-year post-doctoral fellowship at the University of Michigan Medical School (laboratory Prof. P.A. Ward) in Ann Arbor, MI, USA. His research activities related to molecular mechanisms of the inflammatory response in sepsis and acute lung injury, with a particular emphasis on cytokines, complement, and regulatory roles of the apoptotic Fas/FasL signaling system in acute lung inflammation. In 2004, he returned to the University Hospital of Zurich as Senior Physician at the Department of Anesthesiology and the Division of Surgical Intensive Care. His current research is focusing on the inflammatory response in sepsis, lung injury and TBI in the context of volume therapy.

\section{Thomas P. Bleck}

Thomas P. Bleck, MD, FCCM, is the Louise Nerancy Eminent Scholar in Neurology at the University of Virginia and professor of neurology, neurological surgery, and internal medicine. He received his medical degree from Rush Medical College in Chicago, where he also completed residencies in internal medicine and neurology, and a fellowship in epilepsy. Since 1990 he has directed the neuroscience intensive care unit at Virginia. He is certified in internal medicine (with added qualifications in critical care), neurology, and clinical neurophysiology. He is a member of the boards of director of the Society of Critical Care Medicine and the Neurocritical Care Society. He serves as neuroscience editor of Critical Care Medicine, Critical Care, Current Treatment Options in Neurology, and Current Opinion in Critical Care. He is also on the editorial board of Neurocritical Care. Dr Bleck served as the first president of the Neurocritical Care Society from 2003 to 2005. His research interests include head injury, status epilepticus, infectious diseases, acute cerebrovascular diseases, and neuromuscular respiratory failure. He has served more than 100 visiting appointments and lectureships internationally, and has published 75 papers and 119 books and book chapters.

\section{Urban Ungerstedt}

Professor Neuropsychopharmacology, Dept of Physiology and Pharmacology, Karolinska institute, 17177 Stockholm, Sweden. E-mail: urban.ungerstedt@fyfa.ki.se. The present microdialysis method for recovering endogenous neurotransmitters and other chemical substances from the brain was developed in our laboratory and published in 1974. Today about 9000 papers have been published using microdialysis to recover chemical substances from brain as well as peripheral tissues in the body. We have subsequently introduced microdialysis in humans for diabetes monitoring (1989), plastic surgery (1998), neurosurgery and neurointensive care (1990), neonates (2000), liver transplantations (2002) and intraperitoneal monitoring of abdominal ischemia (2003). 


\section{PRESENTING AUTHOR INDEX}

Akavipat, P. Group E: Clinical Practice; 16

Al-Rawi, P. Group F: Experimental Medicine and Pathophysiology; 18

Amadori, A. Group B: Monitoring and Pathophysiology I; 4

Amorim, P. Group E: Clinical Practice; 14

Arango, M. Group E: Clinical Practice; 14

Audibert, G. Group G: Disease Mechanisms; 20

Barcena, J.P. Group C: Clinical Interventions; 7

Batista, S. Group A: Neuroanaesthesia; 2, 3

Belkin, A.A. Group E: Clinical Practice; 15

Bernard, F. Group G: Disease Mechanisms; 21

Bhatia, R. Group G: Disease Mechanisms; 20

Bilgin, H. Group A: Neuroanaesthesia; 1, 4

Bülow, K. Group F: Experimental Medicine and Pathophysiology; 17

Chan, M.T.V. Group F: Experimental Medicine and Pathophysiology; 19

Coles, J.P. Group H: Imaging; 22

Csajbok, L.Z. Group G: Disease Mechanisms; 19

Czosnyka, M. Group D: Monitoring and Pathophysiology II; 10, 11

De Peri, E. Group B: Monitoring and Pathophysiology I; 5

De Peri, E. Group D: Monitoring and Pathophysiology II; 10

Degos, V. Group H: Imaging; 23, 24

DeVito, E.E. Group H: Imaging; 23

Fàbregas, N. Group D: Monitoring and Pathophysiology II; 11

Fernández, S. Group E: Clinical Practice; 14

Ferreira, D.A. Group A: Neuroanaesthesia; 2

Foden, J. Group E: Clinical Practice; 15

Gadgieva, N.S. Group C: Clinical Interventions; 9

Golbækdal, K.I. Group A: Neuroanaesthesia; 1

Golbaekdal, K.I. Group F: Experimental Medicine and Pathophysiology; 16

Goodman, J.C. Group G: Disease Mechanisms; 20

Groefte, T. Group A: Neuroanaesthesia; 1

Groefte, T. Group D: Monitoring and Pathophysiology II; 11, 12

Grousson, S. Group C: Clinical Interventions; 7

Homar, J. Group C: Clinical Interventions; 7, 9

Jensen, L. Group A: Neuroanaesthesia; 2

Jungwirth, B. Group G: Disease Mechanisms; 21

Korfalý, G. Group A: Neuroanaesthesia; 1, 4

Koskinen, L.-O.D. Group F: Experimental Medicine and

Pathophysiology; 18

Krogh, L. Group D: Monitoring and Pathophysiology II; 11, 12
Lavicka, P. Group B: Monitoring and Pathophysiology I; 4 Lavinio, A. Group B: Monitoring and Pathophysiology I; 5 Lescot, T. Group H: Imaging; 23, 24

Lightfoot, R. Group E: Clinical Practice; 13

Lobo, F. Group E: Clinical Practice; 14

Lochhead, V. Group E: Clinical Practice; 15

Lundblad, C. Group G: Disease Mechanisms; 21

Maksimow, A. Group A: Neuroanaesthesia; 3

Møller, K. Group F: Experimental Medicine and Pathophysiology; 17

Møller, K. Group G: Disease Mechanisms; 19

Moretti, M.P. Group B: Monitoring and Pathophysiology I; 4

Nagel, S. Group H: Imaging; 23

Olivecrona, M. Group C: Clinical Interventions; 9

Outtrim, J. Group C: Clinical Interventions; 8

Pedersen, M. Group B: Monitoring and Pathophysiology I; 6

Petrikov, S.S. Group E: Clinical Practice; 13

Poca, M.A. Group B: Monitoring and Pathophysiology I; 5

Rhodes, J.K.J. Group G: Disease Mechanisms; 20

Roman, G. Group A: Neuroanaesthesia; 1

Salmond, C.H. Group H: Imaging; 23

Sato, K. Group F: Experimental Medicine and Pathophysiology; 18

Schmidt, B. Group D: Monitoring and Pathophysiology II; 10

Skoglund, T. Group C: Clinical Interventions; 9

Smielewski, P. Group D: Monitoring and Pathophysiology II; 10, 11

Souter, M.J. Group D: Monitoring and Pathophysiology II; 12

Souter, M.J. Group F: Experimental Medicine and Pathophysiology; 17

Timofeev, I. Group C: Clinical Interventions; 7, 8

Tisdall, M. Group B: Monitoring and Pathophysiology I; 5

Tseng, M.Y. Group C: Clinical Interventions; 8

van Gestel, J.P.J. Group E: Clinical Practice; 16

Vavilala, M.S. Group B: Monitoring and Pathophysiology I; 6

Vittori, C. Group H: Imaging; 22

Wessling, H. Group B: Monitoring and Pathophysiology I; 6

Wolf, S. Group D: Monitoring and Pathophysiology II; 12

Wolf, S. Group F: Experimental Medicine and Pathophysiology; 17

Zavala, E. Group F: Experimental Medicine and Pathophysiology; 16 